\title{
Macroscopic Quantum Tunneling in Josephson Tunnel Junctions and Coulomb Blockade in Single Small Tunnel Junctions
}

\author{
A.N. Clelond \\ Ph.D. Thesis \\ Materials Sciences Division \\ Lawrence Berkeley Laboratory \\ University of California \\ Berkeley, CA 94720
}

April 1991

\section{MASTER}

This work was supported by the Director, Office of Energy Research, Orfice of Basic Energy Sciences, Materials Sciences Division, of the U.S. Department of Energy under Contract No. DE-AC03-76SF00098. 


\section{Macroscopic Quantum Tunneling in \\ Josephson Tunnel Junctions \\ and \\ Coulomb Blockade in \\ Single Small Tunnel Junctions}

by

Andrew Nicholas Cleland

\section{ABSTRACT}

Experiments investigating the process of macroscopic quantum tunneling in a moderately-damped, resistively shunted, Josephson junction are described, followed by a discussion of experiments performed on very small capacitance normal-metal tunnel junctions. The experiments on the resistively-shunted Josephson junction were designed to investigate a quantum process, that of the tunneling of the Josephson phase variable under a potential barrier, in a system in which dissipation plays a major role in the dynamics of motion. All the parameters of the junction were measured using the classical phetiomena of thermal activation and resonant activation. Theoretical predictions are compared with the experimental results, showing good agreement with no adjustable parameters; the tunneling rate in the moderately damped $(Q \approx 1)$ junction is seen to be reduced by a factor of 300 from that predicted for an undamped junction. The phase is seen to be a good quantum-mechanical variable.

The experiments on small capacitance turnel junctions extend the measurements on the larger-area Josephson junctions from the region in which the phase variable has a fairly well-defined value, i.e. its wavefunction has a narrow width, to the region where its value is almost completely 
unknown. The charge on the junction becomes well-defined and is predicted to quantize the current through the junction, giving rise to the Coulomb blockade at low bias. I present the first clear observation of the Coulomb blockade in single junctions. The electrical environment of the tunnel junction, however, strongly affects the behavior of the junction: higher resistance leads are observed to greatly sharpen the Coulomb blockade over that seen with lower resistance leads. I present theoretical descriptions of how the environment influences the junctions; comparisons with the e-perimental results are in reasonable agreement. 


\section{Acknowledgements}

I must first thank Prof. John Clarke, for giving me the chance to work on such interesting experiments and giving me free rein to do as I pleased. I do not think I would have had similar upportunities in any other group. John Martinis introduced me to the world of experimental physics, and taught me much of the lab technique that I know. Fred Wellstood helped to resuscitate the refrigerator on a number of occasions, and was always ready to provide disturbingly accurate descriptions of some piece of physics. I would like to thank John Schmidt, without whose help the second half of this work would have been much more difficult, as well as the members of the Clarke group, for their general support and allowing me to steal their equipment. I thank Y.K. Hui for his careful reading of this thesis.

I am grateful to Prof. T. van Duzer for allowing me to use his SEM lithography system, and to David Chin for helping out at the beginning of the lithography development. I also thank the staff of the Cory Hall Microfabrication Laboratory, for providing a professional and trouble-free work environment. Part of this work was supported by a University of Califomia at Berkeley Fellowship, and I acknowledge the support of an IBM Predoctoral Fellowship. This work was supported by the Director, Office of Energy Research, Office of Basic Energy Sciences, Materials Sciences Division of the U.S. Department of Energy under Contract No. DE-AC0376 SF00098.

I would like to thank my parents, for their support through my long education, and finally I would like to thank my wife, Ning Wang, for her constant love and care. 


\section{TABLE OF CONTENTS}

Chapter 1 Introduction $\ldots \ldots \ldots \ldots \ldots \ldots \ldots \ldots \ldots \ldots \ldots, 1$

Chapter 2 Macroscopic Quantum Tunneling: Theoretical Description 7

2.1 The Resistively Shunted Junction Model ........... 8

2.2 Thermal Activation in the Current-Biased Josephson Junction .......................... 13

2.3 Resonant Activation in the Current-Biased Josephson Junction ............................ 16

2.4 Macroscopic Quantum Tunneling ............. 19

Chapter 3 Macroscopic Quantum Tunneling: Experimental Design . 23

3.1 Junction Environment .................... 24

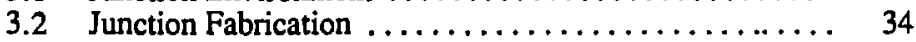

Chapter 4 Macroscopic Quantum Tunneling: Measurements ...... 41

4.1 Measurement of Junction Parameters ........... 42

4.2 Results ............................ 54

Chapter 5 Small Tunnel Junctions: Introduction ............ 59

5.1 Small Tunnel Junctions - Introduction to Theory ....... 60

5.2 Design Considerations .................... 65

Chapter 6 Small Tunnel Junctions: Fabrication and Sample Mount .. 70

6.1 Sample Fabrication ........................ 71

6.2 Single Tunnel Junction Mount ............... 81

6.3 Dilution Refrigerator ...................... 84

6.4 Sample Measurement $\ldots \ldots \ldots \ldots \ldots \ldots \ldots \ldots \ldots, 87$

Chapter 7 Small Tunnel Junctions: Results and Discussion....... 90

7.1 Experimental Results ..................... 91

7.2 Quantum Langevin Theory ................ 99

7.3 Phase Correlation Theory .................. 112

Chapter 8 Conclusions $\ldots \ldots \ldots \ldots \ldots \ldots \ldots \ldots \ldots \ldots, 122$ 


\section{APPENDICES}

Appendix A Capacitance Calculations $\ldots \ldots \ldots \ldots \ldots \ldots \ldots, 127$

Appendix B Resistive Transmission Line $\ldots \ldots \ldots \ldots \ldots \ldots$

Appendix C Electronics $\ldots \ldots \ldots \ldots \ldots \ldots \ldots \ldots \ldots \ldots, 141$

Appendix D Small Junction I-V Characteristics .......... 149

\section{REFERENCES}

References $\ldots \ldots \ldots \ldots \ldots \ldots \ldots \ldots \ldots \ldots \ldots \ldots \ldots, 162$ 


\section{CHAPTER 1}

\section{Introduction}

\section{Macroscopic Quantum Tunneling}

Over the past decade there have been a number of theoretical and experimental investigations of the application of quantum mechanics to electrical circuits, and in particular to Josephson junctions. The fundamental principle on which these investigations are based is that any variable, for which a classical Hamiltonian description exists, can be quantized. In other words, this variable can be described by a quantum wave function and a quantum wave equation corresponding to the quantum Hamiltonian. Common electrical circuit variables such as the charge on a capacitor or the current through an inductor should therefore be quantum variables. However, to test the predictions of quantum mechanics for such a variable, the experimentalist must distinguish between the classical and the quantum behavior of the variable he is measuring. A common example is to create an $L C$ simple harmonic oscillator from the parallel combination of a capacitor $\mathrm{C}$ and an inductor $\mathrm{L}$, and operate it at temperatures low enough that only the ground state of the harmonic oscillator is occupied. It is quite difficult to demonstrate that the oscillator is actually operating in the quantum regime, due to the correspondence limit: all of the excitations of the system are at the oscillator resonance frequency, in both the quantum and classical regimes. This is because the harmonic oscillator is a linear system: The classical equation of motion only involves terms of the first order in the variable of 
interest. It was Leggett who realized that the nonlinearity provided by the Josephson coupling in a Josephson tunnel junction makes it relatively easy to observe the quantum behavior of the Josephson phase variable (1). This coupling transforms the parabolic potential of the $L C$ oscillator into one which includes local minima separated from one another by potential barriers. The Josephson phase variable is the position of an imaginary particle moving in this potential. The Josephson junction is observed to behave in a quantum-mechanical fashion when the phase variable tunnels underneath a potential barrier, leaving a local potential minimum, which causes the junction to switsh from the zero-voltage state to the voltage state. The transition from the classical behavior of the phase variable at high temperatures, to the quantum behavior at low temperatures, is experimentally accessible. The phenomenon of the tunneling of a macroscopic variable, such as the phase of a Josephson junction, which describes the state of all the electrons in the junction, was named "macroscopic quantum tunneling" (MQT) by Leggett (1).

Over the past few years, a number of theoretical works investigating this phenomenon were published $(2,3,4,5,6,7,8)$. The theoretical works progressed from the simple calculation of Ivanchenko and Zil'berman (2) using the Wentzel-Kramers-Brillouin (WKB) technique to calculate the tunneling rate in a non-dissipative system at zero temperature, to the more sophisticated approach of Caldeira and Leggett (3), which allowed the inclusion of weak dissipative effects in the classical and quantum limits. Their work was followed by various calculations involving finite temperatures and strong dissipation. The most interesting aspect of these calculations was the prediction that the presence of dissipation in the classical system would strongly reduce the quantum tunneling rate, by reducing the 
width of the wave function describing the Josephson phase variable: The stronger the dissipation, the narrower the wave function.

Experiments measuring the classical thermal activation rate of Josephson junctions from the zero-voltage state to the voltage state had been carried out earlier, proving the correctness of the detailed classical description $(9,10)$ These experiments furthermore provided the basic techniques used for measurements of the quantum tunneling rate, which are possible at very low temperatures, of the order of $10 \mathrm{mK}$ (temperatures reached by a dilution refrigerator). Measurements of the quantum tunneling rates were carried out by a number of groups $(11,12,13,14,15,16,17)$.

The experimental investigations of quantum tunneling concentrated on experiments in the very lightly damped regime, with the exception of the work of Schwartz et al. (17), which was in the highly damped regime of the RF SQUID. Only the experiments of Devoret $e t$ al. (16) and Schwartz et al. (17) were carried out in a manner which allowed independent measurement of all the parameters. These experiments confirmed the general predictions for the quantum tunneling rate, with the escape rate flattening out at the lowest temperatures. The spectacular observation of quantized energy levels by Martinis et al. (15) provided convincing evidence of the correctness of the quantum approach. There were however some questions about some of the eailier work, due to lack of knowledge of the parameters. Discre pancies appeared in the experimental results of Schwartz et al. (17), which later appeared to be due to problems in the determination of the SQUID parameters. Later adjustments provided excellent agreement with the theory (18).

The work reported here involved measurements on a single Josephson junction, which was fabricated with a parallel metallic resistor to provide a 
moderate level of damping. This work describes the first measurements of the effect of moderate damping on the quantum tunneling rate, and aiso confirms the theories of the escape rate in the regime where quantum tunneling and thermal activation are of equal importance. All the parameters of the junction and its shunt resistor were measured using classical phenomena. Together with the work of Schwartz et al. (17), this work provides convincing experimental evidence of the effect of dissipation on quantum mechanical behavior, in particular of the reduction of the width of the wave function due to damping, observable through the reduction of the quantum tunneling rate from that predicted in the dissipation-free system.

\section{Small Capacitance Tunnel Junctions}

The work on macroscopic quantum tunneling led historically to considerations of what would occur if one made Josephson junctions which are in the quantum regime at all values of bias current; in the MQT experit.lents, the spread in the wave function of the phase variable is much less than $2 \pi$, and therefore quantum effects can be observed only when the phase potential has a nonlinearity over a length much less than $2 \pi$. If one reduces the capacitance of a Josephson junction, it is predicted that the quantum tunneling rate increases, until at some point the phase variable is always tunneling, and its value becomes completely uncertain. The quantummechanical conjugate variable to the phase, the charge on the junction, at that point becomes the "good" quantum variable. This is analogous to the description of an electron, taken from a tight-binding state to a free-running state; the position is the appropriate quantum variable in the former case, but the momentum is appropriate in the latter. 
It was discovered that in this limit a large number of interesting effects should become observable. In the superconducting state the current-voltage (I-V) characteristic was predicted to transform into a radically different form, with frequency-locking effects analogous to the Josephson ac effect (19). In the normal state, with the inclusion of single-electron turneling, the I-V was also predicted to develop striking features, also including frequencylocking effects (20).

A number of experiments were subsequently performed, mostly concentrating on multiple junctions connected in series $(21,22,23,24,25$, 26). The single jurictions in general did not behave in the manner predicted. There were also indications from the multiple junction experiments that the single junctions were being strongly affected by the external circuit, although the detailed mechanism was very unclear. The multiple junction experiments of Delsing et al. (25) and Geerligs et al. (26) were interpreted as being representative of a single junction isolated from its extemal environment, although this is not at all clear from microscopic considerations; the multiple junctions clearly make up a very complex system. The experiment of Fuiton and Dolan (21) showed how a multiple junction setup could be used to channel single electrons through a device, and this was later used to great success by other groups (27).

The experiments reported here were the first to observe the Coulomb blockade in a single small-capacitance tunnel junction, and in doing so I found a simple explanation for the behavior of the single junctions, which takes their extemal environment into account. Other more sophisticated theories were published after this work was first reported. 
The remainder of this thesis is organized as follows: The first half of the thesis will deal with the MQT experiment on the resistively-shunted Josephson junction. Chapter 2 will introduce the resistively-shunted junction (RSJ) model, discuss the classical phenomena of thermal and resonant activation, and summarize the theoretical results for quantum tunneling in the dissipative Josephson junction. Chapter 3 will describe the experimental setup and junction fabrication techniques. In Chapter 4 I give the experimental results and comparisons with theory, and conclude the discussion of MQT. The second lialf of the thesis deals with the smallcapacitance junction experiments. In Chapter 5 I outline the simple theory for the Coulomb blockade and describe the experimental considerations. I describe the experimental setup and junction fabrication in Chapter 6. Chapter 7 describes the experimental results, and I develop a theory for the results using a quantum Langevin approach. I will also present there the gist of the theory of Devoret et al. (28), and point out the possible implications of finite junction tunnel resistance. The conclusions appear in Chapter 8 , following which are the appendices, dealing with technical details of the theory and experiment. 


\section{CHAPTER 2}

\section{Macroscopic Quantum Tunneling: \\ Theoretical Description}

In this chapter I introduce the resistively-shunted-junction (RSJ) model for the Josephson junction, and I discuss the classical phenomena of thermal activation and resonant activation that are used to measure the junction parameters. I then give a brief outline of the principal results of the theories for macroscopic quantum tunneling in the current-biased Josephson junction. 


\subsection{The Resistively Shunted Junction Model}

The resistively shunted junction (RSJ) model of Stewart and McCumber $(29,30)$ has proven to be a reasonably accurate description of the behavior of Josephson tunnel junctions, and is extremely accurate for junctions which have a metallic resistor connected across the junction. The model consists of the electrical circuit shown in Fig. 2.1, a parallel combination of a capacitor $C$, an ideal Josephson element $J$, and a resistor $R$. The circuit has current bias $I$ and includes the resistor current noise $I_{\text {noise- }}$ The Josephson element has voltage $V$ and current $I_{j}$, which obey the two Josephson relations,

$$
\frac{d \delta}{d t}=\frac{2 e}{h} v
$$

and

$$
\mathrm{IJ}_{\mathrm{J}}=\mathrm{I}_{0} \sin (\delta)
$$

where $\delta$ is the Josephson phase variable, equal to the difference in the phases of the two superconducting wave functions on opposite sides of the turnel junction, and $I_{0}$ is the juriction critical current. When the equations for all the circuit elements are written in terms of the phase variable $\delta$ through Eq. (2.1), one obtains the equation of motion for $\delta$,

$$
\frac{\hbar C}{2 e} \frac{d^{2} \delta}{d^{2}}+\frac{\hbar}{2 e} \frac{1}{R} \frac{d \delta}{d t}+I_{0} \sin (\delta)=I+I_{\text {noise }} .
$$




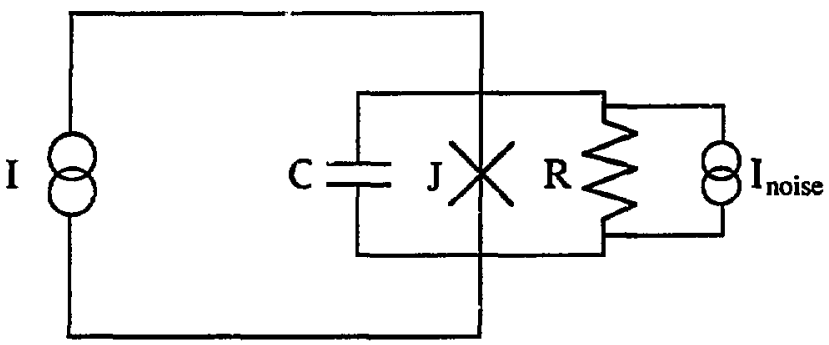

FIGURE 2.1 Resistively shunted Josephson junction. The tunnel junction is modelled as an ideal Josephson element in parallel with an Ohmic resistor and a capacitor, the combination driven by a current bias. The resistor includes a Johnson-Nyquist noise current source. 
This equation of motion is identical to that of a particle with mass proportional to $\mathrm{C}$, moving in a dissipative environment with friction coefficient proportional to $1 / R$, with an external potential $U(\delta)=-\left(\mathrm{I}_{0} \Phi_{0} / 2 \pi\right)$ $\cos (\delta)$ - $\left(\Phi_{0} / 2 \pi\right)$ I $\delta$ (see Fig. 2.2 ); $\Phi_{0}$ is the flux quantum, $\Phi_{0}=\mathrm{h} / 2 \mathrm{e}$. The friction provided by the resistor adds external noise to the system, as a result of the fluctuation-dissipation theorem (31), and this is taken into account through a fluctuating force proportional to $I_{\text {noise }}$.

The general behavior of a junction can be described quite simply with this picture. The zero-voltage state of the junction corresponds to the situation where the phase variable is localized in one of the metastable minima of the potential $U(\delta)$; it oscillates at the resonance frequency $\omega_{p}$ of the local minimum,

$$
\omega_{p}=\sqrt{\frac{2 \pi I_{0}}{\Phi_{0} C}}\left(1-s^{2}\right)^{1 / 4},
$$

where $s=L / T_{0}$ is the current bias; $\omega_{p}$ is called the plasma frequency. The time-averaged voltage $\langle V\rangle$ in this oscillating state is equal to zero, as can be seen from Eq. (2.1). As one increases the bias current above the critical current $I_{0}$, the minimum in which the phase variable rests disappears, and the phase variable begins to run down the potential slope, where $\langle\mathrm{V}\rangle$ is nonzero. As one continues to increase the bias current, the voltage $\langle\mathrm{V}\rangle$ increases, and eventually approaches the linear asymptote $\langle\mathrm{V}\rangle=\mathrm{RI}$. Note that in this running state the instantaneous voltage $V(t)$ includes high frequency components at the Josephson frequency $\omega_{\mathrm{J}}=2 \mathrm{e}<\mathrm{V}>\mathrm{h}$, and all its multiples; these high-frequency components are caused by the undulations in 


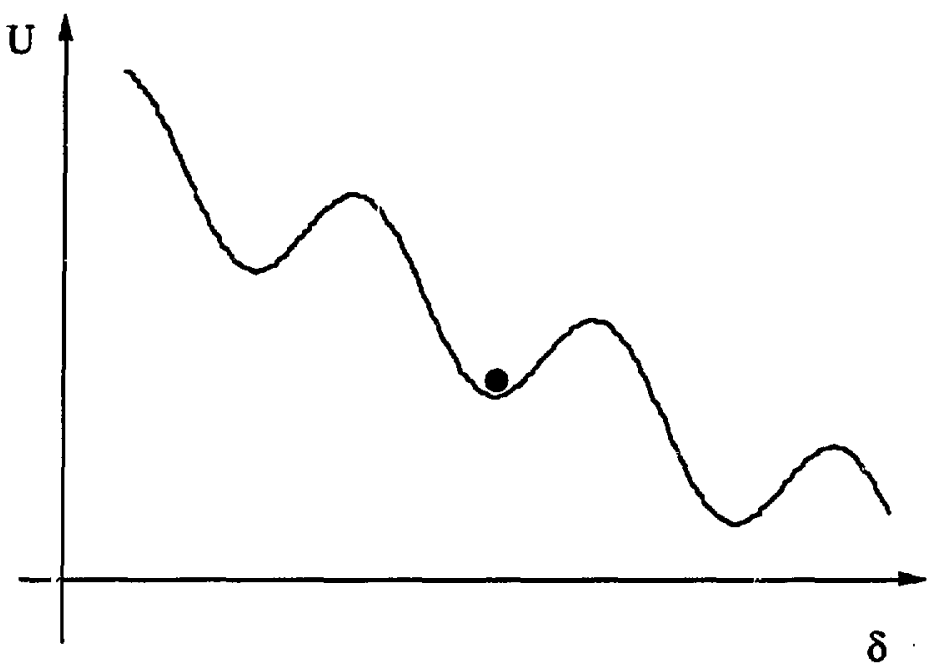

FIGURE 2.2 Tilted washboard potential. The phase particle moves in the washboard potential generated by the Josephson couplin $\tilde{c}_{\mathrm{c}}$ energy and bias current. 
the potential $U(\delta)$, which alternately speed up and slow down the phase variable. In the zero-voltage state, $V(t)$ includes a range of frequencies up to $\omega_{p}$, the frequencies lower than $\omega_{p}$ appearing because of the anharmonic shape of the potential minimum.

The hysteresis observed in the current-voltage characteristics of higher resistance tunnel junctions is easily explained by this model. As one reduces the bias current $I$ below the critical current $I_{0}$, the phase variable will continue to run down the potential, if the dissipative effects of the shunt resistor are small enough. The phase variable will enly retrap in a minimum when the energy gain due to the overall slope of the potential becomes less than the energy lost in the resistor $R$; this occurs at $I \equiv\left(4 \beta_{\mathrm{J}} / \pi\right) I_{0}$, where $\beta_{\mathrm{J}}=$ $1 / \omega_{\mathrm{p}, 0} \mathrm{RC}$ and $\omega_{\mathrm{p}, 0}=\omega_{\mathrm{p}}(\mathrm{s}=0)$.

For smaller values of $R$, the dissipation is greater, and retrapping occurs at larger values of $I$, until at $\beta_{\mathrm{J}} \cong \pi / 4$ the junction $I-V$ is no longer hysteretic. The wide variety of I-V characteristics seen in Josephson tunnel junctions is thus simply explained by this model.

There have been numerous tests of the model described here. In general, the conclusion is that the zero-bias behavior is well described by the RSJ equations, with careful and often empirical choices for the resistor $R$, but that the voltage state usually requires a more careful treatment, due to the unavoidable frequency dependence of the dissipative elements found experimentally. Furthermore, if the value of the resistor $R$ is larger than about $100 \Omega$, it has been clearly shown that the wires used to apply the bias current and measure the voltage across the junction will always short out that resistor, in other words, the parallel impedance of the leads will act to reduce and greatly complicate the effective dissipative element in the model. 


\subsection{Thermal Activation in the Current-Biased Josephson Junction}

One of the sensitive tests of the classical behavior of the phase variable is the measurement of the thermal activation rate of the phase variable out of the metastable minimum, observed by measuring the switching rate of the junction from the zero-voltage state to the voltage state, for bias currents less than the junction critical current $\mathrm{I}_{\mathbf{0}}$. This process was first investigated by Jackel $e t$ al. (9) and Fulton and Dunkleberger (10). If one considers the motion of the phase variable in the bottom of the metastable potential minimum, the current fluctuations in the resistor will maintain the phase variable in thermal equilibrium with the resistor. The probability $\mathrm{P}(\mathrm{E})$ that the phase variable will have energy $E$ in the potential well, given probability $P(0)$ for being at the bottom of the well, is approximately given by $P(E)=P(0) \exp \left(-E / k_{B} T\right)$. This result is only approximate because it ignores the escape process, which depletes the occupation probability at higher energies. One can then intuitively see that the escape rate $\Gamma$ should have the form $\Gamma(T)=(\omega / 2 \pi) \exp \left(-\Delta U / k_{B} T\right)$, where $\omega$ is an attempt frequency and $\Delta \mathrm{U}$ is the barrier height (see Fig. 2.3). It has been shown $(32,33)$ that the exact expression is

$$
\Gamma(T)=a_{c 1}\left(\omega_{p} / 2 \pi\right) \exp \left(-\Delta U / k_{B} T\right)
$$

with barrier height

$$
\Delta \mathrm{U}=\left(2 \sqrt{2} \mathrm{~L}_{0} \Phi_{0} / 3 \pi\right)(1-\mathrm{s})^{3 / 2} \quad(s<<1)
$$




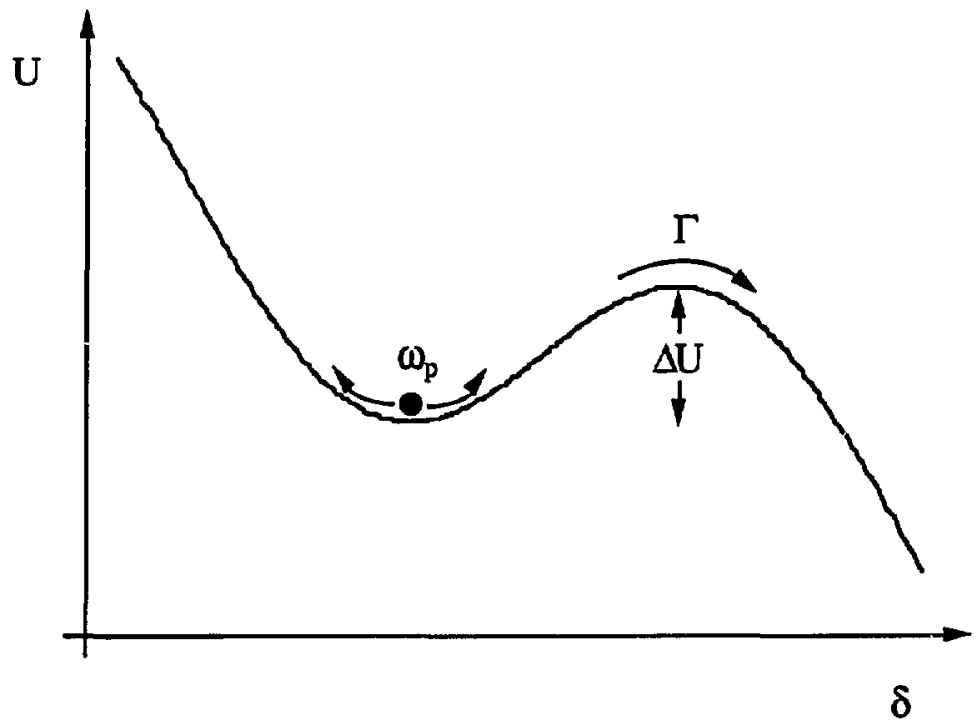

FIGURE 2.3 Parameters describing the motion of the phase particle in a local potential minimum. The particle oscillates at the plasma frequency $\omega_{\mathrm{p}}$ in the bottom of the well, with barrier height $\Delta \mathrm{U}$, and escapes from over the barrier at rate $\Gamma$. 
where $s=I / I_{0}$ is the bias tilt, the attempt frequency is the plasma frequency from the RSJ model given by Eq. (2.4), and $a_{c l}$ is a classical prefactor that takes into account the occupation depletion at high energies mentioned above, $a_{c l}$ has a value near unity. In the range of dissipation applicable to this experiment, $a_{c l}$ has the expression

$$
a_{c l}=\left(1+\frac{1}{4 Q^{2}}\right)^{1 / 2}-\frac{1}{2 Q},
$$

where $Q=\omega_{p} R C$ is the quality factor of the oscillator.

Careful measurements of theimal activation rates in all the damping regimes (extremely underdamped, moderately damped and very lightly damped) agree quite well with Eq. (2.5). Measurements of the escape rate can therefore be used to obtain very precise values of the critical current of a hysteretic Josephson junction; the experiment described here determined $\mathrm{I}_{0}$ to 160 parts per million. This is done by measuring the dependence of the escape rate $\Gamma(I)$ on bias current $I$ at fixed temperature $T$, and plotting the data as $\left[\ln \left(\omega_{p} / 2 \pi \Gamma\right)\right\}^{2 / 3}$ versus $I$; the result is a straight line with an extrapolated intercept at $s=1\left(\mathrm{I}=\mathrm{I}_{0}\right)$ (see Fig. 4.1). As the intercept value depends only logarithmically on $\omega_{p}$, the critical current $I_{0}$ can be determined quite precisely. Independent measurements of the resistance $R$ and capacitance $C$ then allow one to have all three junction parameters well in hand. 


\subsection{Resonant Activation in the Current-Biased Josephson Junction}

Measurements of the resistance and capacitance of a Josephson junction are notoriously difficult. The greatest difficulty comes from the fact that the motion of the phase variable down the washboard potential involves frenuencies near DC as well as frequencies up to the Josephson frequency, which can be as high as $10^{12} \mathrm{~Hz}$. For very low frequencies, the junction is expected to have a low impedance compared to the leads connected across it, and the simple RSJ model should be adequate. However, for motion at frequencies of the order of $10^{9}$ to $10^{12} \mathrm{~Hz}$, the leads connected across the junction can easily provide a shunting impedance of the order of $100 \Omega$, which can be significantly less than the junction's intrinsic impedance; this impedance then becomes the relevant one in the RSJ model. When the experimentalist measures an I-V characteristic, he measures the averaged phase motion at $\mathrm{DC}$; however, some of the dynamics occur at very high frequencies. Extraction of the effective resistance in the RSJ model, usirit only the I-V characteristic, becomes very problematic for high-resistance junctions.

The capacitance is equally difficult to extract (although the currentvoltage characteristic does not yield a value, and therefore is not as distracting as it is for evaluating the junction resistance). One often finds attempts to extract the capacitance from the area of the junction, using values extrapolated from measurements on very large area capacitors with the same dielectric material as the tunnel junction. The difficulty with this approach is that the stray capacitance from structures near the junction, and again from 
the leads connected to the junction, will not be included in the estimate, and make these estimates very unreliable.

A technique was therefore developed which would yield values of the capacitance and resistance of a Josephson junction at the plasma frequency of the junction (34); see also (35). The basic idea is to place the junction in the zero-voltage state, and measure the thermally-activated escape rate; one then applies a weak microwave-frequency current bias in parallel with the constant current bias, and measures the escape rate $\Gamma(\omega)$ as the frequency $\omega$ of the microwave signal is varied. As one sweeps through the plasma frequency $\omega_{p}$ of the junction, one will resonantly couple energy into the junction and therefore measure a peak at a frequency $\omega_{\text {res }}$ in the escape rate $\Gamma(\omega)$; the position of the peak is approximately given by the plasma frequency $\omega_{\mathrm{p}}$, and the width of the peak will be roughly inverse to the quality factor $Q$ of the junction resonance, given by $Q=\omega_{\mathrm{p}} R C$ (see Fig. 4.3). One therefore obtains simultaneous values of the plasma frequency $\omega_{p}$ and the $Q$ of the junction; from these values and the measurement of the critical current Io (see section 2.2), one can calculate values of the junction resistance and capacitance.

Detailed analysis of the resonant activation phenomenon shows it be be quite complex (35); see also references cited therein. The resonance curve above the plasma frequency $\omega_{p}$ cuts off quite sharply, and the width of this side of the resonance is strongly dependent on the value of $Q$; the lower side of the resonance is quite broad and only depends weakly on the value of $Q$. The reason for this is that the shape of the potential well has resonance frequencies below the plasma frequency, as the resonance frequency drops as the energy of the phase particle increases in the well; the plasma frequency corresponds to the zero-energy resonance. The range of these lower 
frequencies depends only weakly on the quality factor. For frequencies above $\omega_{p}$, the phase variable acts just like a harmonic oscillator, and the resonance above $\omega_{p}$ has a width rolighly proportional to $1 / Q$; measurement of the resonance above the plasma frequency therefore gives the $\mathrm{Q}$ of the junction. Numerical simulations are required for more precise results; details are given in the paper cited above.

Analytic treatments of this phenomenon, especially those of Fonseca and Grigolini (36) and Larkin and Ovchinnikoy (37), are in reasonable agreement with the numerical simulations. 


\subsection{Macroscopic Quantum Tunneling}

As one reduces the temperature $\mathrm{T}$ of the junction, holding the barrier height $\Delta U$ constant, the thermal activation theory predicts that the thermaliyactivated escape rate $\Gamma(T)$ will fall exponentially, approaching zero as the temperature approaches zero. However, Caldeira and Leggett $(3,38)$ realized that if one assumes that the phase variable obeys quantum mechanics, then the escape rate should at some point be dominated by quantum tunneling through the potential barrier, and the measured escape rate would then become independent of the temperature.

The basic ideas in the tunneling rate calculation are that the phase variable $\delta$, and its canonically conjugate variable, the charge $q$ on the junction, should obey a commutator relation analogous to that of the position and momentum of a physical particle. Furthermore, it should be poss:ble to model the dissipative effects in the motion of the phase variable by a sufficiently complex Hamiltonian system of equations. Given these two ingredients, Caldeira and Leggett were able to work out an analytic expression for the tunneling rate of the phase variable at $T=0$, in the presence of a small amount of Ohmic dissipation.

The charge $q$ on the junction capacitance $C$ is given by the semiclassical expression

$$
\begin{aligned}
q & =C V \\
& =\frac{\hbar C}{2 e} \frac{d \delta}{d t} .
\end{aligned}
$$


The semi-classical Hamiltonian for the system can then be written in terms of the charge and the phase variables as

$$
\mathrm{H}=\frac{\mathrm{q}^{2}}{2 \mathrm{C}}+\frac{\mathrm{I}_{0} \Phi_{0}}{2 \pi}(\cos \delta-\mathrm{s})+\mathrm{H}_{\mathrm{res}}
$$

where the bias current $\mathrm{I}$ appears as $\mathrm{s}=\mathrm{L} \mathrm{I}_{0}$, and $\mathrm{H}_{\mathrm{res}}$ is the Hamiltonian for the resistor: A workable model for the resistor Hamiltonian is created by replacing the resistor with an infinite set of independent harmonic oscillators, such that the distribution of oscillator frequencies and their coupling to the junction phase variable reproduces the classical behavior of the junction, i.e., so that the velocity-dependent friction term in the phenomenological equation of motion for $\delta$ is reproducsd. The commutator relation between the charge operator $q$ and the phase operator $\delta$ is

$$
[q, \delta]=-.2 e
$$

Using this formalism, after a terrible labor Caldeira and Leggett showed that this zero temperature tunneling rate $\Gamma_{\mathrm{MQT}}$ is approximately given by the expression

$$
\left.\Gamma_{M Q T}=a_{q} \frac{a_{p}}{2 \pi} \exp \left(-7.2 \frac{\Delta U}{\hbar \omega_{p}}(1+0.87 / Q)\right), \quad T=0\right)
$$

with the quantum prefactor $a_{q}=\sqrt{120 \pi\left(7.2 \Delta U / h \omega_{p}\right)}$, barrier height $\Delta U$, junction plasma frequency $\omega_{p}$, and quality factor $Q$. This expression holds for low damping, $Q>5$. In the region of interest for the experiment 
described here, with $Q \cong 1$, analytic expressions are not easily available, and one resorts to the numerical results given by Grabert et al. $(39,40)$.

There are two very interesting effects described by Eq. (2.11). One is the well-known effect of quantum tunneling; in the limit of a dissipation-free system $(Q=\infty)$, one obtains the WKB expression for the tunneling rate (2). The observation of a simple quantum effect, such as tunneling, in the phase variable of a Josephson junction is truly remarkable. One can believe that any classical variable, including as it does here the charge on an electrical device, should in some limit behave quantum mechanically. In other words, there is no reason that quantum mechanins should only describe the behavior of the position and momentum of particles such as electrons or atoms.

The other fascinating result of the calculation of Caldeira and Leggett (3) is that one finds that dissipative systems have smaller tunneling rates than non-dissipative ones; smaller values of $Q$ lead to lower tunneling rates. This is often said to imply that the wave function of a particle in a dissipative environment becomes less spread out. This result therefore may shed some light, although only in a very vague way, on some of the fundamental questions relating to measurement theory in quantum mechanics: If one assumes that the measurement apparatus is extremely dissipative, then it will behave classically, and the measurement process is then a classical one.

The crossover between quantum tunneling and thermal activation occurs at the temperature $T_{c r}$, which is given in terms of the plasma frequency $\omega_{\mathrm{p}}$ by

$$
k_{B} T_{c r}=\frac{\hbar \omega_{p}}{2 \pi}\left[\sqrt{1+\alpha^{2}}-\alpha\right]
$$


where $\alpha=1 / 2 \mathrm{Q}$. Well above this temperature, the escape from the zerovoltage state occurs primarily through thermal activation, and as one reduces the temperature towards $T_{c r}$ the quantum effects become more noticeable. Below $T_{c r}$ the escape is dominated by tunneling effects, with temperature dependence at low $\mathrm{T}$ given by

$$
\ln (\Gamma(T) / \Gamma(0)) \propto T^{2}
$$

Several authors report measurements of the temperature dependence of the tunneling rate $(12,13,14,15,17,41)$.

The work described here has been reported elsewhere (42). In the next section I discuss the experimental details, and the results are described in a later section. 


\section{CHAPTER 3}

\section{Macroscopic Quantum Tunneling: \\ Experimental Design}

In this chapter I describe the experimental setup used to measure the MQT escape rate. The design philosophy was to use a junction and a junction mount which could be simply characterized in terms of the resistivelyshunted-junction (RSJ) model, so that the classical and quantum predictions given in the previous chapter could be applied with confidence. 


\subsection{Junction Environment}

\section{Junction Mount}

The experimental setup used for the MQT measurements has been extensively described elsewhere (41). The junction mount was designed so that the impedance, from the point of view of the junction connected to it, would be that of a resistor with an impedance of about $200 \Omega$, but the mount would include dissipative elements which would absorb high-frequency signals, and therefore eliminate reflections from the ends of the mount. These two design aims were met by putting the junction on the end of a coaxial line, with a $\mathrm{Cu}$ tube forming the outer conductor and a $\mathrm{Nb}$ wire forming the center conductor. The space between the $\mathrm{Cu}$ and the $\mathrm{Nb}$ wire was filled with a mixture of Stycast 2850 epoxy and fine Cu powder $(50 \mu \mathrm{m}$ diameter). The $\mathrm{Cu}$ powder in the coaxial line serves as a microwave attenuator: The large surface area of the individual $\mathrm{Cu}$ grains acts to absorb signals, but a native oxide on the grain surface isolates one grain from the other. The $\mathrm{Cu}$ powder begins absorbing microwave signals above about 0.2 $\mathrm{GHz}$, and absorbs signals up to at least $12 \mathrm{GHz}$. The Stycast epoxy ensures that the $\mathrm{Cu}$ powder is in thermal equilibrium with the dilution refrigerator.

This mount assembly had a reasonably flat electrical impedance, equal to about $200 \Omega$ above $1 \mathrm{GHz}$ with no obvious resonances, and a signal attenuation of about $10 \mathrm{~dB}$ above $1 \mathrm{GHz}$. The design therefore met the dual purpose described above. In order to couple weak microwave signals into the junction, a microwave injection line was capacitively coupled to the $\mathrm{Nb}$ center conductor, placed about half-way along the length of the $\mathrm{Cu}$ rod. This coupling gave about $60 \mathrm{~dB}$ of attenuation, and therefore provided a currentbias microwave signal. 


\section{Microwave Filters}

In order to attenuate signals at frequencies below $1 \mathrm{GHz}$, where the junction mount is transparent to spurious signals, standard RC filters with a 3 $\mathrm{dB}$ point at about $1 \mathrm{MHz}$ were also used. This standard type of filter however only works below about $1 \mathrm{GHz}$. Additional filtering above $1 \mathrm{GHz}$ was provided by assemblies consisting of wire spirals potted in $\mathrm{Cu}$ powder and Stycast epoxy. These microwave filters, in addition to the $\mathrm{RC}$ filters, were placed in series with the junction mount. A set of filters was placed in the liquid ${ }^{4} \mathrm{He}$ bath, and another set bolted to the mixing chamber. The combined filter stages gave a total attenuation of about $200 \mathrm{~dB}$ above $100 \mathrm{MHz}$. A schematic wiririg diagram is shown in Fig. 3.1. A separate set of filters was built for use on a $4.2 \mathrm{~K}$ cryostat for resonant activation measurements.

\section{Switching Electronics}

The electronics used to measure the switching distribution of the junction has been described elsewhere (41). The method worked as follows: The current to the junction was provided by a voltage ramp, and the current was measured with a custom-built differential amplifier connected across a bias resistor. A schematic for the amplifier is shown in Appendix C. The current ramp was passed into the refrigerator cryostat, through the RF and microwave filters, and then through the junction. The refrigerator was used as the electrical ground for the current return and the voltage signals. The junction voltage was amplified by a Brookdeal 5004 low-noise preamplifier, and in turn by a PAR 113 amplifier. When the junction switched to the voltage state, the voltage was detected by the trigger of a custom-built 12-bit A-to-D converter, causing the A-to-D converter to digitize the value of the 


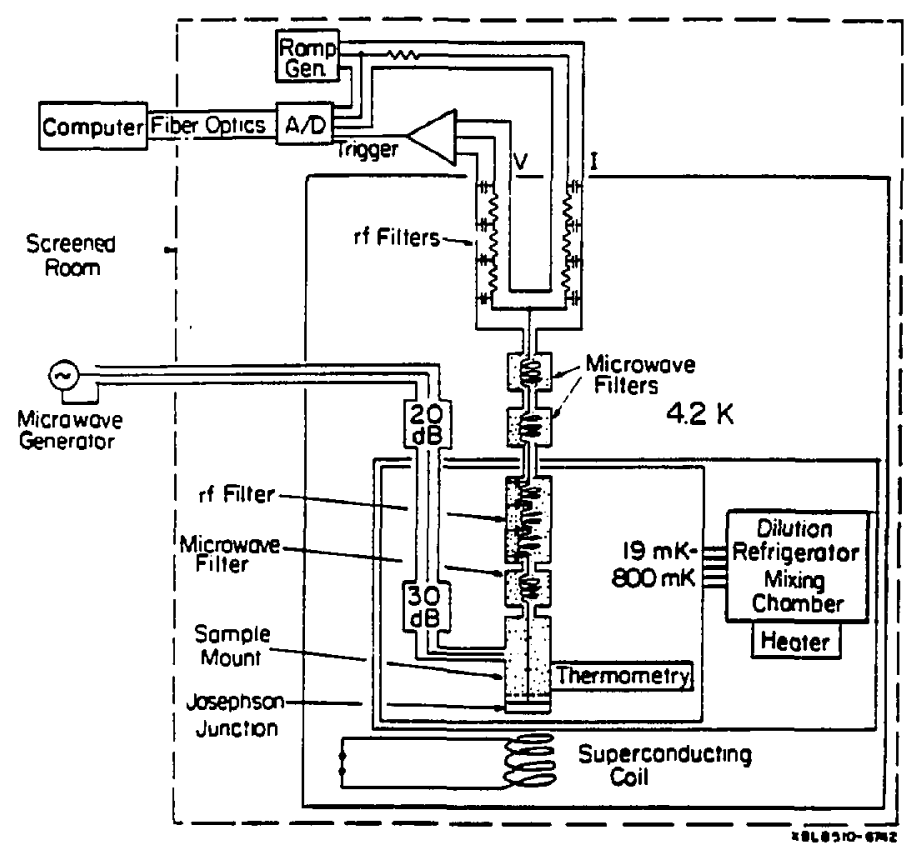

FIGURE 3.1 Schematic of the wiring used for the MQT measurements. 
current passing through the junction at that moment. The digitized value of current was then sent along a set of optical fibers to an LSI-11 computer, where the data was stored in a histogram and subsequently manipulated. The voltage signal from the junction was also used to shut off the current ramp biasing the junction; this was done in order to prevent excessive heating of the junction and its mount once the junction was in the voltage state (see below). Following this, another current ramp would begin for another escape rate measurement.

The data taken with this set-up consisted therefore of a histogram of switching events as a function of bias current, taken at a fixed refrigerator temperature. An example of such a histogram is shown in Fig. 3.2.

The current ramp, the A-to-D converter and all the amplifiers were powered by $12 \mathrm{~V}$ car batteries and placed inside a screened room with the dilution refrigerator. The computer was placed outside the screened room with plastic optical fibers linking it to the A-to-D converter. There was therefore no explicit shared ground between the computer and the other electronics. A schematic is shown in Fig. 3.3.

\section{Data Manipulation}

The method of calculating the escape rate dependence on bias current from the histogram of switching events has been described elsewhere (10). Given a histogram with $N\left(I_{n}\right)$ switching events at a bias current $I_{n}$, the escape rate $\Gamma\left(I_{n}\right)$ is given by

$$
\Gamma\left(I_{n}\right)=\frac{d I}{d t} \frac{1}{\Delta I} \ln \left(\sum_{j=1}^{n} N\left(I_{j}\right) / \sum_{i=1}^{n-1} N\left(I_{i}\right)\right),
$$


where $d \mathrm{l} / \mathrm{dt}$ is the current ramp rate and $\Delta \mathrm{I}$ is the histogram bin width; bin 1 is the bin at the highest value of bias current. plot of the switching rate as calculated from the histogram of Fig. 3.2 is shown in Fig. 3.4. Switching distribution histograms typically had $10^{4}$ events, and statistical checks on the distributions were used to ensure proper operation of the setup. Because of junction heating problems (see below), up to 0.5 seconds was allowed to elapse after switching off the junction bias current and before beginning another current ramp. A histogram therefore could take over an hour to accumulate. Care had to be taken to keep the refrigerator temperature stable over this period of time.

\section{Dilution Refrigerator}

The dilution refrigerator used in this experiment consisted of an Oxford Model 75 dilution unit, capable of reaching temperatures below 20 $\mathrm{mK}$, and a homemade insert and gas handling system (the latter was built by John Schmidt and Mark Ferrari). Peripheral to the refrigerator were the ${ }^{4} \mathrm{He}$ $1 \mathrm{~K}$ pot system, a thermometry rack, and a vacuum system for the refrigerator vacuum can.

The dilution refrigerator had a circulation rate of about $60 \mu \mathrm{moles} / \mathrm{sec}$ of $90 \%{ }^{3} \mathrm{He} / 4 \mathrm{He}$ when it was operating at its base temperature of $18 \mathrm{mK}$. The cooling power was about $1 \mu \mathrm{W}$ at $25 \mathrm{rKK}$. The principal difficulty with this refrigerator was that the needle valve which regulated the flow of liquid ${ }^{4} \mathrm{He}$ into the $1 \mathrm{~K}$ pot would become clogged after about 10 days of operation, and the flow rate would become increasingly difficult to regulate after that. We made several attempts to solve this problem, but as of this writing the problem remained. 


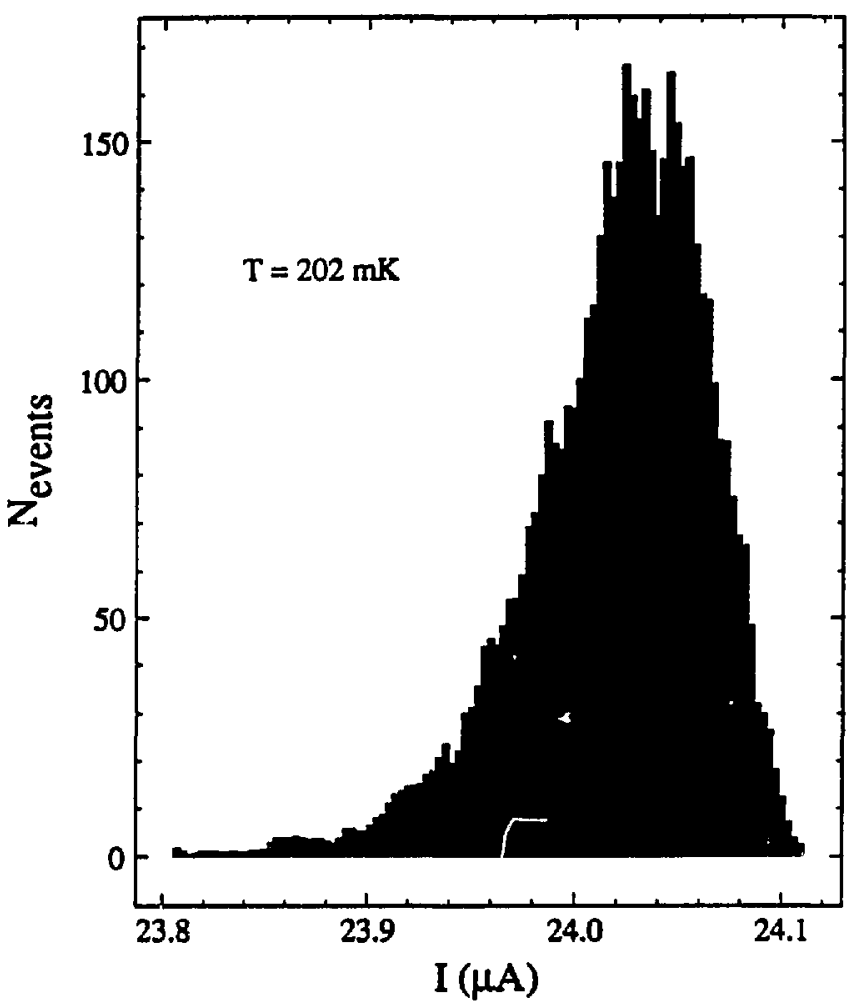

FIGURE 3.2 Histogram of number of switching events $\mathrm{N}_{\text {events }}$ as a function of bias current $I$, taken at a temperature $T=202 \mathrm{mK}$. 


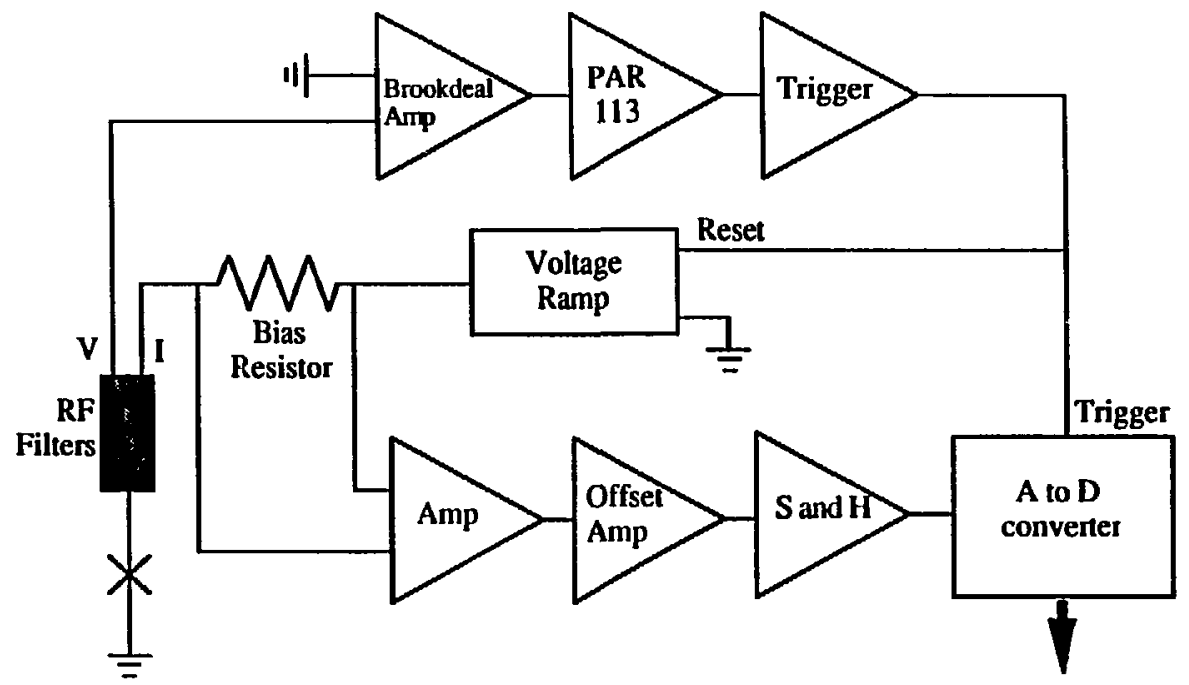

To LSI-11

FIGURE 3.3 Schenatic of electronics used to measure the value of bias current at which the junction would switch frcm the zero-voltage state to the voltage state. 


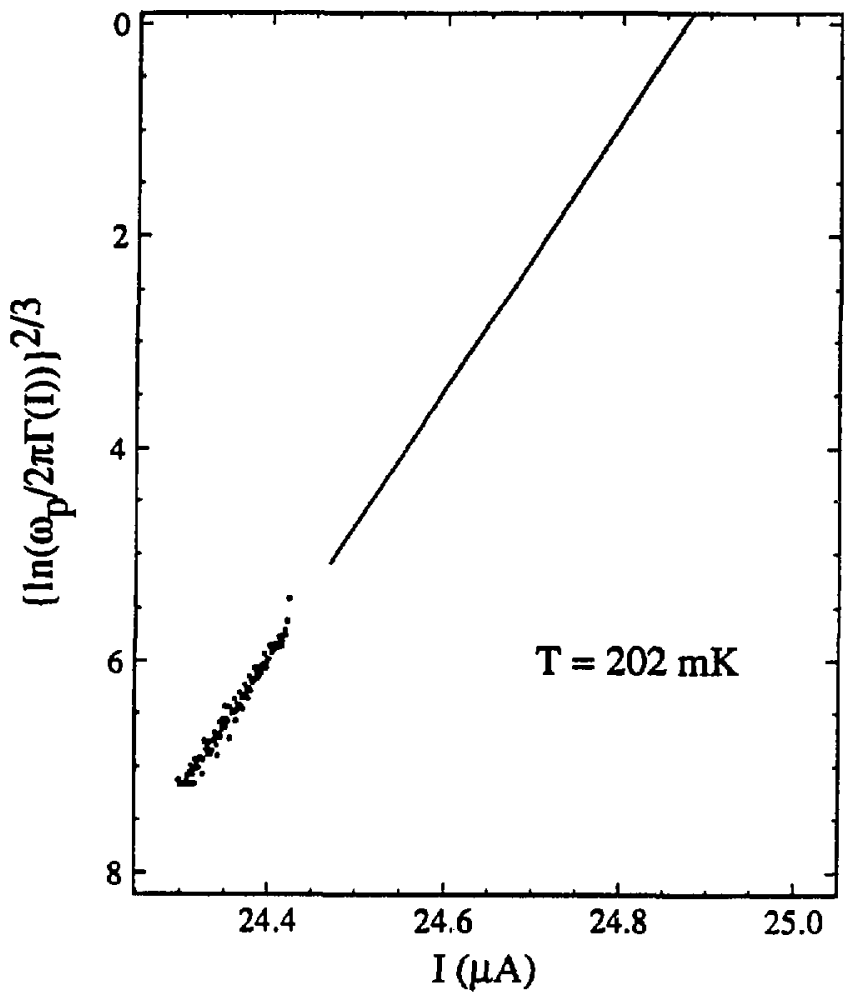

FIGURE $3.4\left\{\ln \left(\omega_{p} / 2 \pi \Gamma(I)\right)\right\}^{2 / \beta}$ as a function of bias current I, calculated from the histogram of Fig. 3.2 using Eq. (3.1). The refrigerator temperature is $\mathrm{T}=202 \mathrm{mK}$. The straight line intercept on the current axis at $24.876 \mu \mathrm{A}$ is the first-order determination of the critical current $\mathrm{I}_{0}$. 


\section{Thermometry}

The thermometry on the refrigerator included a set of roughly calibrated carbon resistors on the $1 \mathrm{~K}$ pot, the still, the plate and the mixing chamber, and two more carefully calibrated resistors on the sample mount: a $\mathrm{Ge}$ resistance thermometer (Lakeshore), which was calibrated from $300 \mathrm{~K}$ to about $60 \mathrm{mK}$, and a carbon resistor (made by Matsushita Co.) calibrated from $300 \mathrm{~K}$ to $18 \mathrm{mK}$. The Ge resistor and the other carbon resistors on the refrigerator were monitored with a Lakeshore conductance bridge. The Matsushita resistor was monitored with a homemade resistance bridge, which was also capable of regulating the refrigerator temperature to within about $0.5 \mathrm{mK}$ by feedback heating of a metal film resistor on the mixing chamber.

The Ge resistance thermometer was calibrated using data taken by Fred Wellstood (43): A dc SQUID was used to measure the current noise generated in a $71 \mathrm{~m} \Omega$ resistor, and the magnitude of this noise used to calibrate the $\mathrm{Ge}$ thermometer, whose resistance was measured simultaneously. The carbon resistor was prepared by grinding down a 100 $\Omega$ Matsushita resistor to a thickness of about $500 \mu \mathrm{m}$ and clamping it in a $\mathrm{Cu}$ mount. The ground-down resistor had a room-temperature resistance of 220 $\Omega$, which rose to about $60 \mathrm{k} \Omega$ at $18 \mathrm{mK}$. It was calibrated against the $\mathrm{Ge}$ thermometer above $70 \mathrm{mK}$, and calibrated from $20 \mathrm{mK}$ to $60 \mathrm{mK}$ using a ${ }^{60} \mathrm{Co}$ nuclear orientation thermometer. The ${ }^{60} \mathrm{Co}$ thermometer is an absolute thermometer, and the method of use is described by Lounasmaa (44). A piece of ferromagnetic single crystal Co (provided by Prof. N.E. Phillips of the Chemistry Department) was attached to the mixing chamber, and the rate of $\gamma$-ray emission from the ${ }^{60} \mathrm{Co}$ nuclei was measured along the magnetic crystal axis. The radiation pattem is temperature-dependent, and the ratio of the emission rate at the unknown temperature to the rate at some temperature 
higher than $60 \mathrm{mK}$ (I used $120 \mathrm{mK}$ ) gives one an absolute temperature calibration. The higher temperature need not be known with any precision. Measurements at a number of points between $18 \mathrm{mK}$ and $60 \mathrm{mK}$ gave a resistance calibration accurate to about $0.5 \mathrm{mK}$.

\subsection{K Measurements}

The resonant activation experiments and precise I-V measurements were carried out in a $4.2 \mathrm{~K}$ cryostat. A mechanical pump was used to reduce the temperature down to $1.2 \mathrm{~K}$, and the bath pressure used as a thermometer. The junction remained on the mount described above, and was not disturbed between measurements at $4.2 \mathrm{~K}$ and measurements on the dilution refrigerator. A different set of $\mathrm{RF}$ and microwave filters from that on the refrigerator was used, but tests of these filters indicated they had over $100 \mathrm{~d} \overline{\mathrm{s}}$ of attenuation above $100 \mathrm{MHz}$, easily adequate for measurements down to 1.2 $K$. The microwave source used for the resonant activation measurements consisted of a set of backward wave oscillators (Hewlett-Packard Co.) which together covered the span of frequencies from 1 to $12 \mathrm{GHz}$. The microwave signals were coupled through the capacitive injection point on the junction mount. The same A-to-D converter and computer were used for the measurements at $4.2 \mathrm{~K}$ as for the measurements on the refrigerator, with equal care to avoid grounding problems. 


\subsection{Junction Fabrication}

The junctions used for this experiment were fabricated on $5.08 \mathrm{~cm}$ diameter, $<100>$ orientation Si wafers, $0.35-0.5 \mathrm{~mm}$ thick. The Si was ptype, with a room-temperature resistivity of 1 to $5 \Omega-\mathrm{cm}$. The Si acted as a substrate for the junction fabrication, and a $1000 \mathrm{~nm} \mathrm{SiO}{ }_{2}$ layer was thermally grown on the Si surface to provide electrical isolation. I describe first some of the basic steps followed in the fabrication procedure, and then discuss the detailed fabrication sequence used to produce a complete, resistively-shunted, junction.

\section{Basic Fabrication Procedures}

1. Wafer cleaning: The wafers were soaked in detergent (RBS-35 detergent from the Pierce Chemical Co.) for 20 minutes and rir.sed with deionized (DI) water.

2. Photoresist coating: The wafers were heated briefly in a $70^{\circ} \mathrm{C}$ oven to drive off residual water vapor, and were then exposed to vapor saturated with hexamethyl disilazane (HMDS, from the Merck Chemical Co.), a photoresist adhesion promoter, for 5 minutes. They were then placed on a photoresist spinner, and one of two resist solutions was placed on the surface of the wafer. For etch patterns I used Shipley 1450J resist spus at $5000 \mathrm{rpm}$ for 30 seconds, and for liftoff pattems I used Shipley 1400-31 spun at 6000 rpm for 30 seconds. The wafers were then placed on a hotplate for 5 minutes, either at $70^{\circ} \mathrm{C}$ for the $1450 \mathrm{~J}$ resist or at $90^{\circ} \mathrm{C}$ for the $1400-31$ resist. The resist then typically had a thickness of 1.2 to $1.5 \mu \mathrm{m}$. If the resist was intended for a liftoff pattem, a good undercut on the pattem edges was desired, and this was accomplished by soaking the baked wafers in a $18-20^{\circ} \mathrm{C}$ 
chlorobenzene bath for 10 minutes, followed by a second 5 minute bake on a $90^{\circ} \mathrm{C}$ hotplate.

3. Pattern definition: The baked wafers were placed on the exposure system (a $4 \times$ reduction Canon FPA-141 mask aligner), and exposed with the mask pattern. The exposure pattem consisted of nine $11 \mathrm{~mm} \times 11 \mathrm{~mm}$ dies, which were independently aligned. For liftoff pattems I used an exposure setting of 4.9 and an $\mathrm{f}$-stop of 1.4 , while for etch patterns I used an exposure of 5.6. The exposed pattern was developed out in Microposit Developer Concentrate (Shipley Co.), either full strength with a 10-15 second immersion for the liftoff pattems, or diluted 1:1 with water and a 50 second immersion for the etch pattems. The wafer was rinsed with DI water and checked under an optical microscope.

4. Evaporations: materials were evaporated in a Varian 3118 evaporator, which consisted of an oil diffusion pump backed by a mechanical pump, both of which had $\mathrm{LN}_{2}$ cold traps at the inputs. Base pressure was about $7 \times 10^{-7}$ Torr, attainable after about 1 hour of pumping. All the materials were evaporated by resistive heating of a $W$ boat (or in the case of SiO, a W oven), with deposited thickness measured with a quartz crystal resonator. The boats were purchased from R.D. Mathis Co., and the crystals and crystal monitors were from the Kurt J. Lesker $\mathrm{Co} . \mathrm{Nb}$ is a refractory metal and was deposited by magnetron sputtering (see below). Liftoff of undesired material was accomplished by soaking the wafer in acetone until all the resist was gone, followed by a second acetone soak for 5 minutes. Etching is described below. 


\section{Detailed Junction Fabrication}

1. Resistor fabrication: the wafers were cleaned, photoresist processed for liftoff, and placed in the evaporator. The resistors for this experiment were to be about $10 \Omega$, and this value of resistance was easily fabricated using a CuAu alloy ( $25 \mathrm{wt}$. \% Cu). A measured quantity of $\mathrm{Cu}$ and $\mathrm{Au}$ (13 pellets of 10 mesh $\mathrm{Cu}$ shot, and a 1/4" length of $1 / 16^{\prime \prime}$ diameter Au wire) was placed in a $W$ boat, and the wafers were placed face down on a holder attached to the top plate of the evaporator. The spacing from the wafer to the boat was about $30 \mathrm{~cm}$, with an intervening shutter that was manually controlled with a rotating feedthrough in the top plate. In order to ensure that the resistor material would adhere to the wafer, a $\mathrm{Cr}$ layer was evaporated first from a $\mathrm{W}$ boat. The $\mathrm{Cr}$ and $\mathrm{CuAu}$ were evaporated after a 20 minute pumpdown, the $\mathrm{Cr}$ at a rate of about $0.2 \mathrm{~nm} / \mathrm{sec}$ for a total thickness of $2-3 \mathrm{~nm}$, and the $\mathrm{CuAu}$ at a rate of about $1 \mathrm{~nm} / \mathrm{sec}$ for a total thickness of about $35 \mathrm{~nm}$. This was followed by a liftoff. The deposited material had a resistivity of about $4 \Omega$ per square, and the lithography pattern was designed so that the resistor value could be chosen at fabrication time.

2. $\mathrm{Nb}$ base electrode fabrication: The wafers were cleaned, and placed in a homemade sputter system which consisted of an oil diffusion pump backed by a mechanical pump. The diffusion pump had a cold trap at the inlet, and the mechanical pump had a molecular sieve trap. The base pressure was about $1 \times 10^{-6}$ Torr. The top plate of the stainless steel chamber had a magnetron sputter gun with an annular target (made by Sloan) mounted in the center, and the wafer sat in a shuttered compartment about $4 \mathrm{~cm}$ below the $\mathrm{Nb}$ sputter target. After a 20 minute pumpdown, Ar gas was bled in at a pressure of about 5 to $10 \mathrm{~m}$ Torr, and a plasma ignited using a $360 \mathrm{~V} \mathrm{DC}$ 
voltage bias across the sputter gun anode and cathode. Once ignited, the plasma typically drew about $4 \mathrm{~A}$. After a 2 minute presputter, the shutter was opened abruptly and sputtering continued for $\mathbf{2 0}$ seconds, at which point the voltage bias was shut off. This procedure resulted in a $200 \mathrm{~nm}$ thick $\mathrm{Nb}$ layer, with a superconducting transition temperature of about $9 \mathrm{~K}$. The $\mathrm{Nb}$ coated wafer was then coated with photoresist and pattemed for an etch process. Etching was done in a plasma barrel reactor, using an $\mathrm{SF}_{6} \mathrm{O}_{2}$ plasma driven by a $180 \mathrm{~W}$ RF source, at an $\mathrm{SF}_{6} \mathrm{O}_{2}$ pressure of about 0.4 Torr.

3. SiO deposition: The wafers were cleaned, and photoresist processing was done for a liftoff. The SiO was evaporated in the Varian evaporator, with the same geometry as for the CuAu evaporations. The SiO was purchased from the R.D. Mathis Co., and a few small chunks were placed in a W evaporation oven (also from R.D. Mathis). A 2-3 nm thick layer of $\mathrm{Cr}$ was evaporated first to promote adhesion. Typical SiO deposition rates were about $1 \mathrm{~nm} / \mathrm{sec}$ with a total thickness of $200 \mathrm{~nm}$.

4. Junction oxidation and PbIn top electrode deposition: The wafers were cleaned and patterned for a liftoff. As the oxidation step was notoriously unreliable, the wafers at this point were diced up into the nine 11 $\mathrm{mm} \times 11 \mathrm{~mm}$ chips for individual processing (each chip had a single shunted junction and an unshunted junction, the latter used for checking junction quality). The dicing was done by hand-scribing the wafer using a diamondtipped scribe, and breaking the wafer across the edge of a glass slide. The wafers had been patterned so that the chip edges were aligned with the $\mathrm{Si}$ crystal axes. This choice of pattem alignment allows this crude dicing method to work. 
A single patterned chip was placed under an Ion Tech ion mill gun, which was mounted on a top plate for the Varian 3118 evaporator. The chip would be ion milled to remove the native oxide layer on the $\mathrm{Nb}$ base electrode, which would otherwise be too thick for Josephson tunneling. After a 20 minute pumpdown, the gun was tumed on in an Ar pressure of about $10 \mathrm{mT}$ Trr, with a $300 \mathrm{~V}$ bias and a current density of about 0.5 $\mathrm{mA} / \mathrm{cm}^{2}$. The chip was ion milled for 40 seconds, and the ion gun was then tumed off. The system was bled to atmospheric pressure with dry $\mathrm{N}_{2}$, and the chip was removed to the oxidation/PbIn mount, which consisted of a large anode plate bolted to an evaporator top plate, used with the 3118 evaporator. $\mathrm{A} \mathrm{W}$ evaporation boat with $\mathrm{Pb}$ wire and a small quantity of In wire ( 5 wt. \% In) had been previously placed in the evaporator, and the system was again pumped out. This step was done as rapidly as possible, as exposing the now clean $\mathrm{Nb}$ base electrode to air causes the $\mathrm{Nb}$ to form a oxide layer of uncontrollable thickness and quality.

After pumping for 20 minutes, the junction was oxidized by bleeding in an Ar- $\mathrm{O}_{2}$ mixture $\left(10 \% \mathrm{O}_{2}\right)$ at a pressure of about $7 \mathrm{mTorr}$. A plasma was ignited in the chamber using a Hartley oscillator operating at $7 \mathrm{MHz}$, with amplification provided by an RCA 813 vacuum tube. The oxide thickness was controlled by varying the oxidation time, with typical oxidation times of $40-50$ seconds. Finally the $\mathrm{Pb}$-In mixture was evaporated to completion, giving a top electrode of about $200 \mathrm{~nm}$ thickness with a superconducting transition temperature of about $8 \mathrm{~K}$.

\section{Junction layout}

The pattern layout of the junctions was designed to give some user flexibility in choosing the junction area (with base electrode width of either 5 
or $10 \mu \mathrm{m}$ ) and in resistor value (anything from 2 to 20 squares). The $\mathrm{CuAu}$ resistor was ground-planed by the $\mathrm{PbIn}$ top electrode. The SiO layer provided electrical isolation between the PbIn groundplane and the $\mathrm{CuAu}$ resistor. A schematic layout is shown in Fig. 3.5. Typical junction parameters were junction critical current $I_{0}$ of $10-30 \mu \mathrm{A}$, junction shunt resistance $R$ of 5 to $20 \Omega$, and junction capacitance $C$ of 5 to $10 \mathrm{pF}$. Lead connections were made using blobs of In dabbed onto $1 \mathrm{~mm}^{2} \mathrm{Nb}$ pads on the chip periphery.

In the process of developing this experiment, several junctions were made and run through various parts of the measurement. However, all the results presented in the next chapter are for a single, shunted junction. 


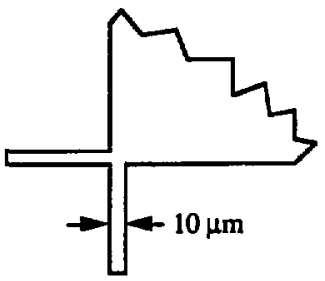

1. CuAu resistor with $1 \mathrm{~mm}^{2}$ cooling fin, $30 \mathrm{~nm}$ thick.

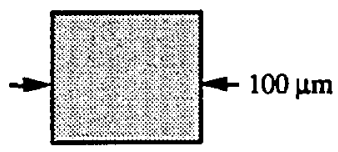

3. SiO insulation layer, 200 nm thick.

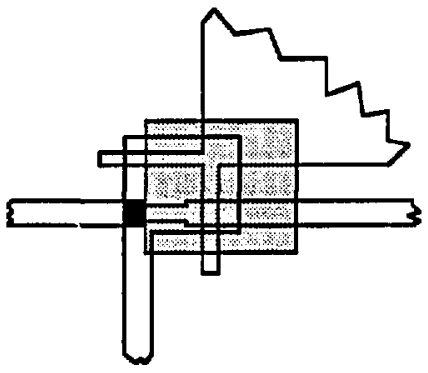

5. Junction assembly

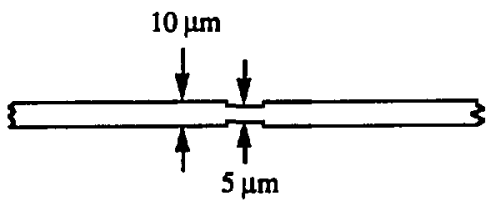

2. $\mathrm{Nb}$ base electrode, $200 \mathrm{~nm}$ thick.

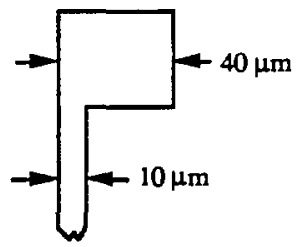

4. PbIn top electrode, $150 \mathrm{~nm}$ thick.

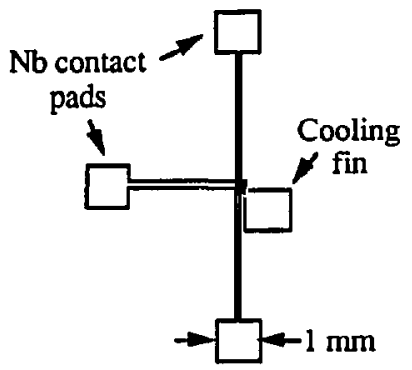

6. Chip layout, showing contact pads and cooling fin.

FIGURE 3.5 Junction fabrication steps for the resistively shunted junction. A detailed description is given in the text. 


\section{CHAPTER 4}

\section{Macroscopic Quantum Tunneling: \\ Mersurements}

In this chapter I describe the various measurements of the junction parameters, give the results of those measurements, and then present the data from measurements of the switching rate of the phase variable from the zerovoltage state. These data cover a range from the highest temperatures, where the classical theory of ther: I activation applies, to the lowest temperatures, where quantum turneling was observed to occur. 


\subsection{Measurement of Junction Parameters}

In this section I summarize the results of the various parameter measurements performed on the junction, both in the $4.2 \mathrm{~K}$ cryostat and on the dilution refrigerator.

\section{Escape Temperature}

Measurements of the switching distribution of the junction from the zero-voltage state to the voltage state at a temperature $T$ are parameterized by the escape temperature $T_{\text {esc }}$, defined by the relation

$$
\Gamma(T)=\frac{\omega_{p}}{2 \pi} \exp \left(-\Delta U / k_{B} T_{e s c}\right) \text {. }
$$

where $\Gamma(T)$ is the measured escape rate. In the limit of high temperatures $T$, the escape temperarure $T_{\text {esc }}$ is approximately equal to $\mathrm{T}$ (the relation is only approximate because of the classical prefactor $\mathrm{a}_{\mathrm{cl}}$ appearing in Eq. (2.5), which is not equal to unity). For the junction described here, it is found that at high $\mathrm{T}, \mathrm{T}_{\text {esc }}=0.985 \mathrm{~T}$. As one reduces the temperature $\mathrm{T}$ below the crossover temperature $T_{c r}$ (see Chapter 2 ), the escape temperature flattens and approaches a non-zero limit at $\mathrm{T}=0$.

\section{Critical Current $I_{0}$}

Measurement of the critical current $I_{0}$ of the junction is performed by measuring the escape rate $\Gamma$ at high temperatures $T \gg T_{c r}$. Measurements of the dependence of the escape rate on the bias current I allow the extraction of the critical current. From Eq. (2.5) and (2.6), we see that a plot of 


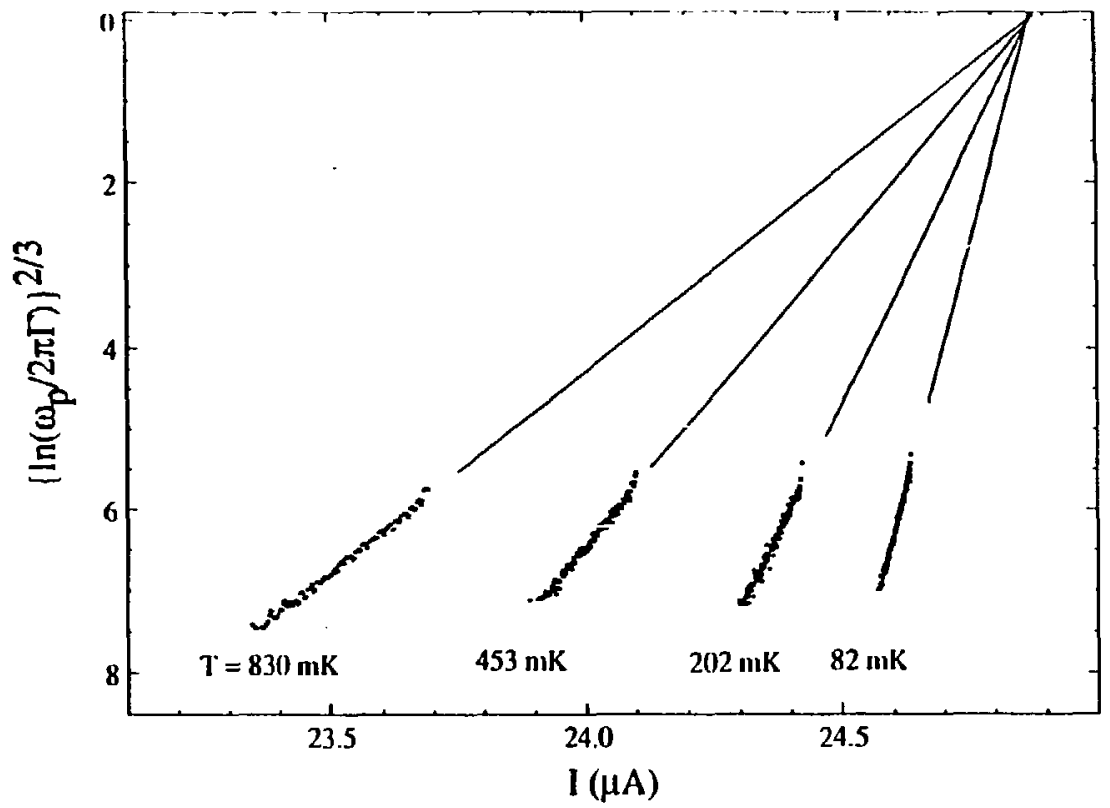

FIGURE 4.1 Ploi of $\left\{\ln \left(\omega_{p} / 2 \pi \Gamma(1)\right)\right\}^{2 / 3}$ as a function of bias current 1 , for four different temperatures $T$. Each set of points represents $10^{4}$ escape events. The first-order critical current $\mathrm{I}_{0}(\mathrm{I})$ extracted from the data is shown by the straight line extrapolation (see text). 
$\left(\ln \left[\omega_{\mathrm{p}}(\mathrm{I}) / 2 \pi \Gamma(\mathrm{I})\right]\right\}^{2 / 3}$ as a function of $\mathrm{I}$ should be a straight line, with slope proportional to $T_{\text {esc, }}$ and with intercept on the current axis at $I \cong I_{0}$ (the equality would be exact if $a_{c l}=1$ ). An example of this measurement in shown in Fig. 4.1. The graphically determined critical current $\mathrm{I}_{0}(1)$ is corrected for the actual prefactor $a_{c l}$, yielding the current $I_{0}(2)$; quantum corrections can then be applied to yield the current $\mathrm{I}_{0}(3)$. A tabulation of these values as a function of temperature appears in Table 4.1. In this experiment, the classically determined critical current $\mathrm{I}_{0}(2)$ was used to interpret the lower temperature switching data, so that no assumptions concerning the quantum state would be used. The critical current of the junction was found to depend weakly on temperature for $\mathrm{T}>1 \mathrm{~K}$, and below $1 \mathrm{~K}$ was independent of $\mathrm{T}$. The value of the critical current $I_{0}$ was determined to be $24.873 \pm 0.004 \mu \mathrm{A}$ below $1 \mathrm{~K}$, obtained by averaging the values of $\mathrm{I}_{0}(2)$ fo: the four highest temperatures appearing in Table 4.1. For temperatures below $300 \mathrm{mK}$ the quantum corrections were deemed too large to be ignored. Above $1 \mathrm{~K}$, where the critical current was needed to interpret the resonant activation data, Io was measured prior to each resonant activation measurement.

\section{Capacitance $C$}

The value of the capacitance of the junction was determined by the position of the peak in the resonant activation experiment. Such a measurement at $4.2 \mathrm{~K}$ is shown in Fig. 4.2. The analytic function given in Devoret et al. (35) is plotted as the solid line in that figure. The fitting parameters $\lambda=5$ and $c=3.2$ used to give the solid line (see the reference $p$. 70) correspond to a value of $Q=2.5$. The position of the resonant activation peak at $9.0 \mathrm{GHz}$ corresponds to a capacitance of $4.0 \mathrm{pF}$, giving a resistance 


\begin{tabular}{ccccc}
\hline $\begin{array}{c}\text { Temperature } \\
(\mathrm{mK})\end{array}$ & $\begin{array}{c}\mathrm{I}_{0}(1) \\
(\mu \mathrm{A})\end{array}$ & $\begin{array}{c}\mathrm{I}_{0}(2) \\
(\mu \mathrm{A})\end{array}$ & $\begin{array}{c}\mathrm{I}_{0}(3) \\
(\mu \mathrm{A})\end{array}$ & $\begin{array}{c}\sigma \\
(\mu \mathrm{A})\end{array}$ \\
\hline 830 & 24.895 & 24.880 & 24.882 & 0.012 \\
627 & 24.900 & 24.887 & 24.890 & 0.010 \\
453 & 24.882 & 24.871 & 24.875 & 0.007 \\
333 & 24.877 & 24.867 & 24.872 & 0.006 \\
267 & 24.870 & 24.861 & 24.867 & 0.004 \\
202 & 24.876 & 24.868 & 24.876 & 0.003 \\
158 & 24.868 & 24.861 & 24.871 & 0.002 \\
126 & 24.869 & 24.862 & 24.874 & 0.002 \\
104 & 24.867 & 24.861 & 24.875 & 0.002 \\
93 & 24.864 & 24.858 & 24.873 & 0.002 \\
82 & 24.859 & 24.853 & 24.870 & 0.002 \\
\hline
\end{tabular}

TABLE 4.1. Measured critical current, as extracted from the escape rate measurements. The first column is the dilution refrigerator temperature, as measured with the carbon resistor thermometer. The second column, $\mathrm{I}_{0}(1)$, is the value of critical current obtained using a classical prefactor $\mathrm{a}_{\mathrm{cl}}=1$; the column $I_{0}(2)$ is obtained using the correct classical prefactor, and the column $\mathrm{I}_{0}(3)$ is obtained using the prefactor including quantum corrections. $\sigma$ is the standard deviation in the measurement. 


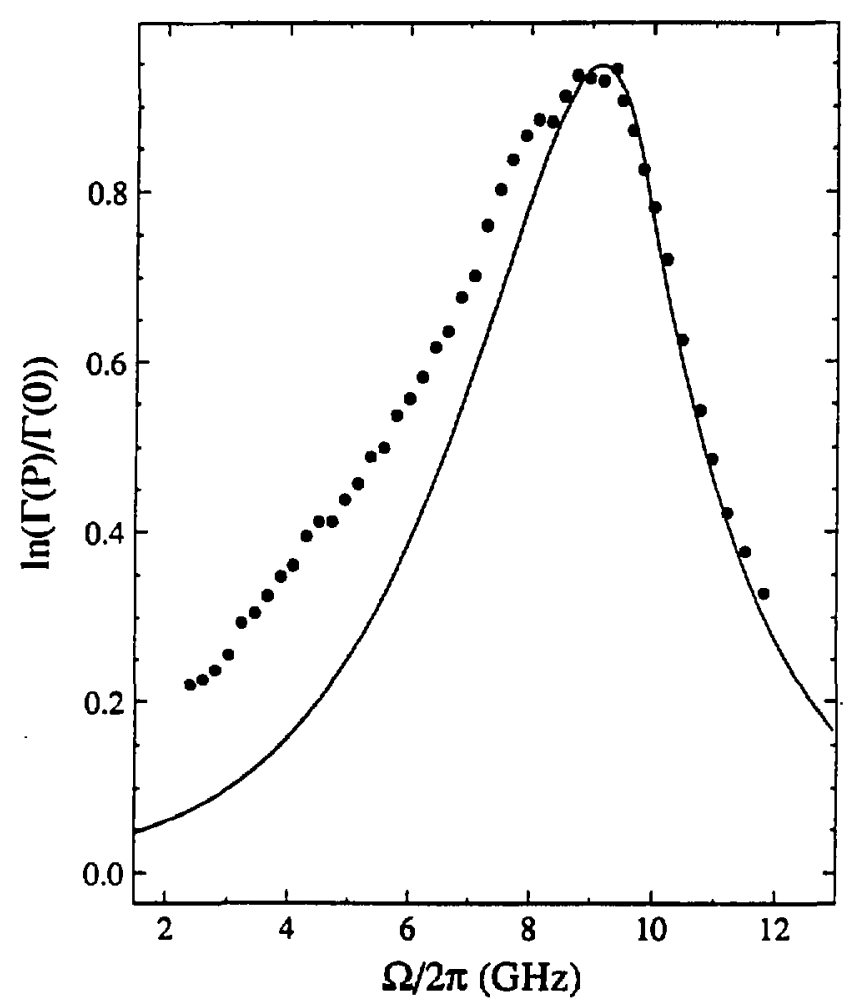

FIGURE 4.2 Resonant activation of current-biased Josephson junction. The data (solid dots) show the logarithm of the ratio of the escape rate with microwave power $\Gamma(P)$ to that without microwave power $\Gamma(0)$, plotted as a function of microwave frequency $\Omega / 2 \pi$. The theoretical curve (solid line) is calculated from Devoret et al. (35) 
$R=10 \Omega$. Several resonant activation measurements were performed, at different values of critical current and temperature. From these measurements, the capacitance of the junction was determined to be $\mathrm{C}=4.28 \pm 0.34 \mathrm{pF}$ for a nominal junction area of $50 \mu \mathrm{m}^{2}$. This value is in good agreement with that expected for $\mathrm{Nb}$ oxide, where the measured ratio of oxide thickness $t$ to oxide dielectric constant $\varepsilon$ is $t / \varepsilon=0.1 \mathrm{~nm}$ (45).

\section{Resistance $R$}

The resistance $R$ of a shurted junction can be determined in two ways: from the current-voltage characteristic, or from the width of the resonance peak in the resonant activation experiment. The former measurement was done in a $4.2 \mathrm{~K}$ cryostat, using carefully calibrated current and voltage amplifiers and tracing the I-V characteristic on an X-Y recorder. The I-V is shown in Fig. 4.3. The value of $R$ measured from the slope of the I-V for large I gives the result $R=9.3 \pm 0.1 \Omega$. The resonant activation measurement gives a value that is much less precise than this value, as there are large uncertainties in the theory for junctions with low quality factor $Q$. Fitting the shape of the resonance for frequencies above the resonance frequency $\omega_{\text {res }}$ gave the result $R=14 \pm 9 \Omega$, in reasonable agrement with the I-V measurement. It is important that the activation experiment be performed, as it has been shown that for unshunted junctions with resistances larger than about $100 \Omega$ (this is the resistance below the superconducting gap voltage $2 \Delta / \mathrm{e}$ !), the leads are the principal dissipative mechanism at the plasma frequency. The junction mount used in this experiment was shown to have an effective shunting re "istance of about $200 \Omega$ (41), well above the net shunt resistance of $9.3 \Omega$, so this should not be a problem. 


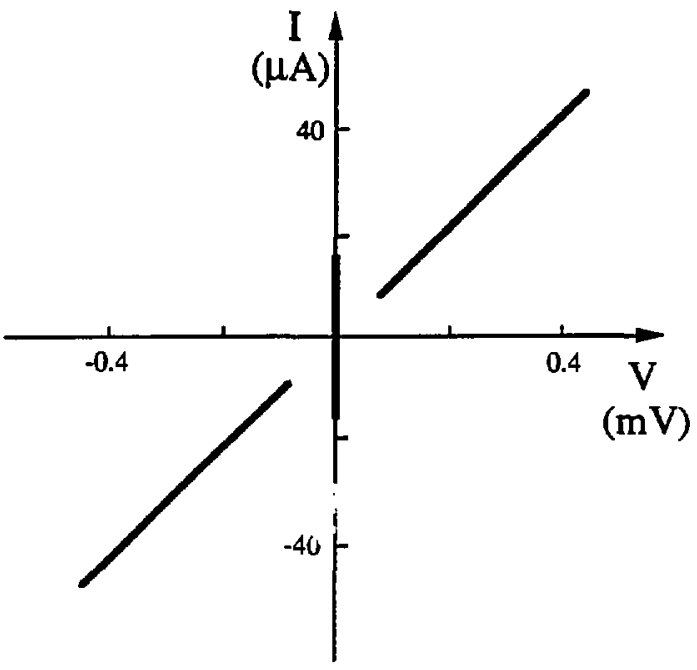

FIGURE 4.3 Current-voltage characteristic measured at $4.2 \mathrm{~K}$. The critical current is reduced from its $\mathrm{T}=0$ value of $\mathrm{I}_{0}=24.9 \mu \mathrm{A}$, but the resistive shunt has the temperature-independent value of $R=9.3 \Omega$. The shunt resistance was measured from the I-V characteristic. 
A summary of the values determined from these measurements is given in Table 4.2.

\section{Heating Effects}

Measurements of the escape rate at the lowest dilution refrigerator temperatures indicated that heating in the shunt resistor was a serious problem. Heat is dissipated in the resistor once the junction switches into the free-running state, where a finite voltage appears across the junction. After resetting the junction to the zero-voltage state, the resistor will begin cooling, but the cooling rate was found to be excessively long. An experiment was devised to measure the cooling rate of the resistor. The junction would be switched into the voltage state for $20 \mu \mathrm{s}$, in order to heat up the resistor, following which the junction would be reset to the zero-voltage state and an escape rate ineasurement would be performed a time $\tau_{\text {cool later, where }} \tau_{\text {cool }}$ varied from $20 \mu \mathrm{s}$ to $1 \mathrm{~ms}$ following the initial pulse. I present the data in Fig. 4.4 as the excess escape temperature, defined as $T_{\text {excess }}=T_{\text {esc }}$ (with heat pulse) $-\mathrm{T}_{\text {ece }}$ (no heat pulse), plotted against the cooling time $\tau_{\text {cool }}$. The escape temperature with no heat pulse was measured with 1 second elapsing between escape events. It was found that for a refrigerator temperature of $20 \mathrm{mK}$, $T_{\text {excess }}$ as measured $40 \mu \mathrm{s}$ after the heat pulse would be about $90 \mathrm{mK}$, and even $1 \mathrm{~ms}$ after the heat pulse the excess temperature would still be about $20 \mathrm{mK}$. This was determined to be a hot-electron effect: the electrons in the shunt are in poor thermal equilibrium with the phonons, and non-equilibrium heating of the electron gas leads to a long thermal time constant for the small volume of the shunt resistor. The coupling between the electrons and phonons follows the equation $(43,44)$ 


\begin{tabular}{cccccc|ccc}
\hline $\begin{array}{c}\mathrm{I}_{0} \\
(\mu \mathrm{A})\end{array}$ & $\begin{array}{c}\mathrm{C} \\
(\mathrm{pF})\end{array}$ & $\begin{array}{c}\mathrm{R} \\
(\Omega)\end{array}$ & $\begin{array}{c}\mathrm{I} \\
(\mu \mathrm{A})\end{array}$ & $\begin{array}{c}\omega_{\mathrm{p}} / 2 \pi \\
(\mathrm{GHz})\end{array}$ & $\mathrm{Q}$ & $\begin{array}{c}\mathrm{T} \\
(\mathrm{mK})\end{array}$ & $\begin{array}{c}\mathrm{T}_{\text {esc }} \\
(\text { meas.) }\end{array}$ & $\begin{array}{c}\mathrm{T}_{\text {esc }} \\
(\text { pred })\end{array}$ \\
\hline 24.873 & 4.28 & 9.3 & 24.710 & 7.2 & 1.77 & 18 & $46 \pm 2$ & $45 \pm 2$ \\
\pm 0.004 & \pm 0.34 & \pm 0.1 & & \pm 0.3 & \pm 0.07 & 0 & $45 \pm 2$ & $43 \pm 2$ \\
\hline
\end{tabular}

Table 4.2 Junction parameters and the measured and predicted values of $T_{\text {esc }}$ at a refrigerator temperature of $T=18 \mathrm{mK}$, as well as the extrapolated values of $T_{\text {esc }}$ at $T=0$, from the experiment and theory. $I$ is the bias current at which $\omega_{p}, Q$ and $T_{e s c}$ were evaluated. 


$$
\frac{\mathrm{P}}{\mathrm{V}}=\Sigma\left(\mathrm{T}_{\mathrm{c}}{ }^{5}-\mathrm{T}_{\mathrm{ph}}{ }^{5}\right)
$$

where the power $P$ per volume $V$ of electron gas heats the electrons to a temperature $T_{e}$ for a phonon temperature $T_{p h} . \Sigma$ is a constant involving the electron-phonon coupling constant, and for $\mathrm{Cu}$ has been evaluated to be $\Sigma=$ $2 \times 10^{9} \mathrm{~W} / \mathrm{m}^{3}-\mathrm{K}^{5}$.

The time dependence of the electron gas temperature $T_{c}(t)$ can be estimated using Eq. (4.2). Using the low-temperature formula for the electron heat capacity $Q_{e}=\gamma T_{e}^{2}(46)$, and assuming the only power loss is through electron-phonon interactions, we find that the electron temperature should follow the equation

$$
2 \gamma \Gamma_{e} \frac{d T_{e}}{d t}=-\Sigma\left(T_{e} 5-T_{p h}{ }^{5}\right)
$$

With the assumption that $T_{e} \gg T_{p h} I$ drop $T_{p h}$ in Eq. (4.3) and solve to find

$$
T_{e}(t)=\left(\frac{1}{T_{e}^{3}(0)}+\frac{3 \Sigma}{2 y} t\right)^{-1 / 3}
$$

The value $T_{\text {excess }}=T_{e}(t)-T_{p h}$ is plotted in Fig. 4.4 as a solid line, using the value of $\Sigma$ above and the value of $\gamma$ for $\mathrm{Cu}$ (as the value for the $\mathrm{CuAu}$ alloy is not known). The phonon temperature was $T_{p h}=25 \mathrm{mK}$. The comparison with the cooling curve for the shunt resistor without a cooling fin is fairly good; considering the lack of knowledge of the parameters, the agreement is perhaps fortuitous. 


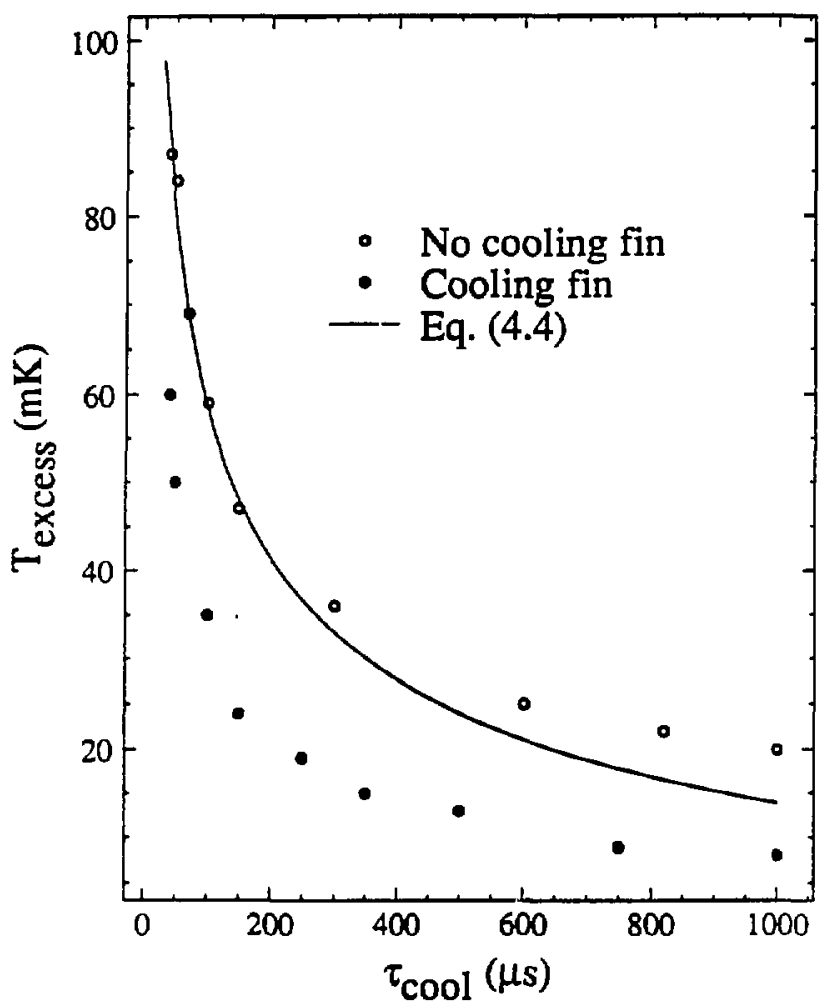

FIGURE 4.4 Excess escape temperature $T_{\text {excess }} v s$ cooling time $\tau_{\text {corl }}$. A heat pulse was applied by putting the junction in the voltage state for $20 \mu \mathrm{s}$. After resetting the junction to the zero voltage state at $\tau_{\text {cool }}=0, T_{\text {exc }}$ was measured with a delay $\tau_{\text {cooll }}$. The excess escape temperature is defined as $T_{\text {excess }}=$ $T_{\text {esc }}$ (with heat pulse) $-T_{\text {esc }}$ (no heat pulse). The refrigerator temperature was about $25 \mathrm{mK}$. The open circles are for a shunt resistor with no cooling fin, the solid circles with a cooling fin; the solid line is the prediction of Eq. (4.4). 
In order to solve this heating problem, a large $1 \mathrm{~mm}^{2}$ cooling fin was attached to the shunt resistor, thus increasing the volume of the electron gas without significantly changing the resistor value. Bare Si wafers were also used, instead of the wafers with the insulating $1000 \mathrm{~nm}$-thick $\mathrm{SiO}_{2}$ layer. The heat pulse measurement described above showed that $10 \mu$ following the initial pulse, the electron gas heated to an excess temperature of $60 \mathrm{mK}$, and cooled in $1 \mathrm{~ms}$ to an excess temperature of $9 \mathrm{mK}$, significantly better than without the cooling fin (see Fig. 4.4). Note that the cooling fin makes the cooling a non-equilibrium process, and Eq. (4.4) would not be expected to apply.

Measurements of the switching distribution at the lowest refrigerator temperatures were done with a $300 \mathrm{~ms}$ interval between escape events, even using the cooling fin, in order to ensure that heating was not a problem. 


\subsection{Results}

The results of the measurement of the escape temperature $T_{\text {esc }}$ as a function of $T$ are shown in Fig, 4.5. Note that the value of bias current, at which the junction switches most often, changes with temperature; the barrier height $\Delta \mathrm{U}$ is not the same at all points in the plot. It is clearly seen that for $T \gg T_{c r}$ the data follow the classical prediction quite closely, while as the temperature is reduced to below $T_{c r}$ the escape temperature flattens and approaches a zero-temperature limit. The theoretical curve for $T>T_{c r}$ is calculated from Eq. (11) of Grabert and Weiss (6), while for $T<T_{c r}$ the numerical expressions of Grabert, Olschowski and Weiss are used $(39,40)$. The theoretical and experimental curves are in good agreement over the entire range of experimental temperatures. In Figs. 4.6 and 4.7 I show the detailed behavior of the escape temperature above and below $T_{c r}$. The data in Fig. 4.6 are seen to follow a $T^{2}$ dependence quite well, over a wide range of temperatures, but the measured slope is seen to be about $30 \%$ smaller than the prediction. The data are always seen to be within a factor of two of the theoretical data. Note that if I vary the junction parameters used to obtain the predicted curve, the theoretical prediction shifts uniformly with little change in slope; in other words, the slope of the $\mathrm{T}^{2}$ dependence is roughly independent of the junction parameters, while the magnitude is quite sensitive to those parameters.

In order to test the behavior of the junction above the crossover temperature $T_{c r}$, the enhancement of the escape temperature over the actual temperature is shown in Fig. 4.7. The data are seen to be in excellent agreement with the predictions of the classical theory including quantum corrections, and diverges strongly from the purely classical theory with no 
corrections. At $T=T_{c r}$ the quantum corrections have increased $T_{c r}$ to a value $40 \%$ larger than the thermal value.

The intent of this experiment was to observe quantum tunneling in a Josephson junction, and by using a low-Q junction observe the effect of dissipation on the quantum tunneling rate. It is clearly seen that both of these effects have been observed: for temperature down to the crossover temperature $T_{c r}$, the quantum corrections are clearly necessary to explain the data, and for measurements of the escape rate at the lowest temperatures the observed rates are far higher than can be explained with the classical theory. It can furthermore be seen from Fig. 4.6 that the measured escape rate, extrapolated to $T=0$, is a factor of 300 smaller than the rate expected for an identical junction with infinite shunt resistance $(Q=\infty)$, in good agreement with the theory. This shows the strong effect of the finite value of the dissipative element on the quantum tunneling rate. 


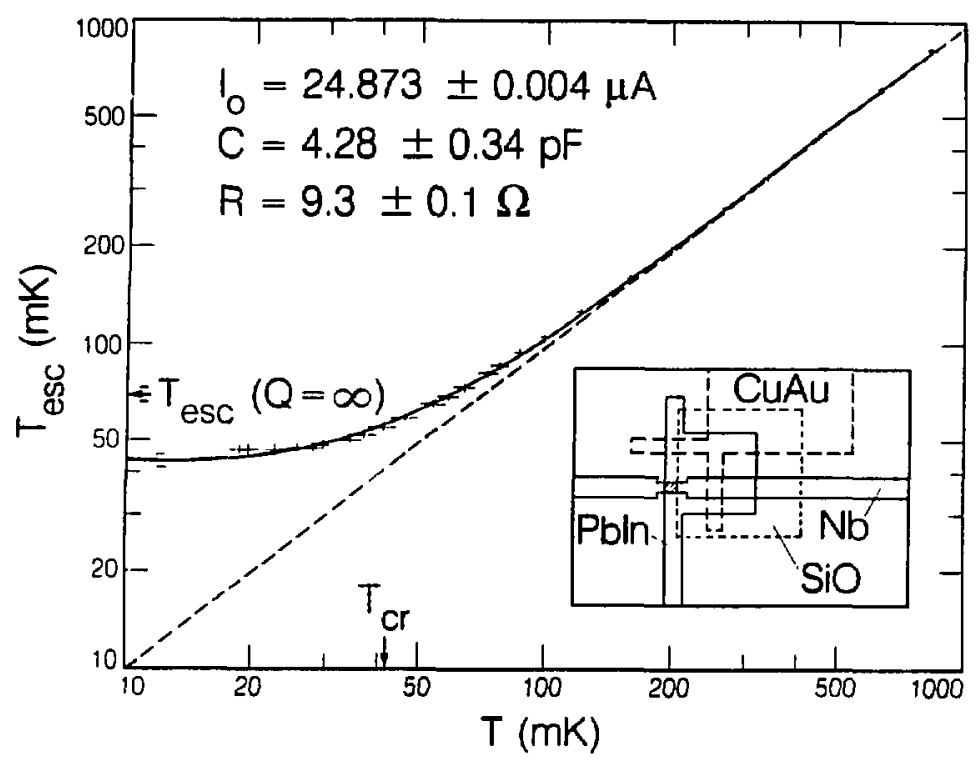

FIGURE 4.5 Escape temperature $T_{e x}$ as a function of refrigerator temperature T. At high temperatures the data follow the classical prediction $T_{e s c}=0.985 \mathrm{~T}$, while at low temperatures the data flatten out due to quantum tunneling. Vertical error bars indicate uncertainty in the junction parameters, and the horizontal error bars indicate systematic uncc.tainty in the temperature calibration. The solid curve is the prediction of theory, with the horizontal error bars indicating uncertainty due to the junction parameters. The inset shows the junction configuration. The crossover temperature $T_{r}$ and the $T=0$ limit of the infinite- $Q$ system are indicated. 


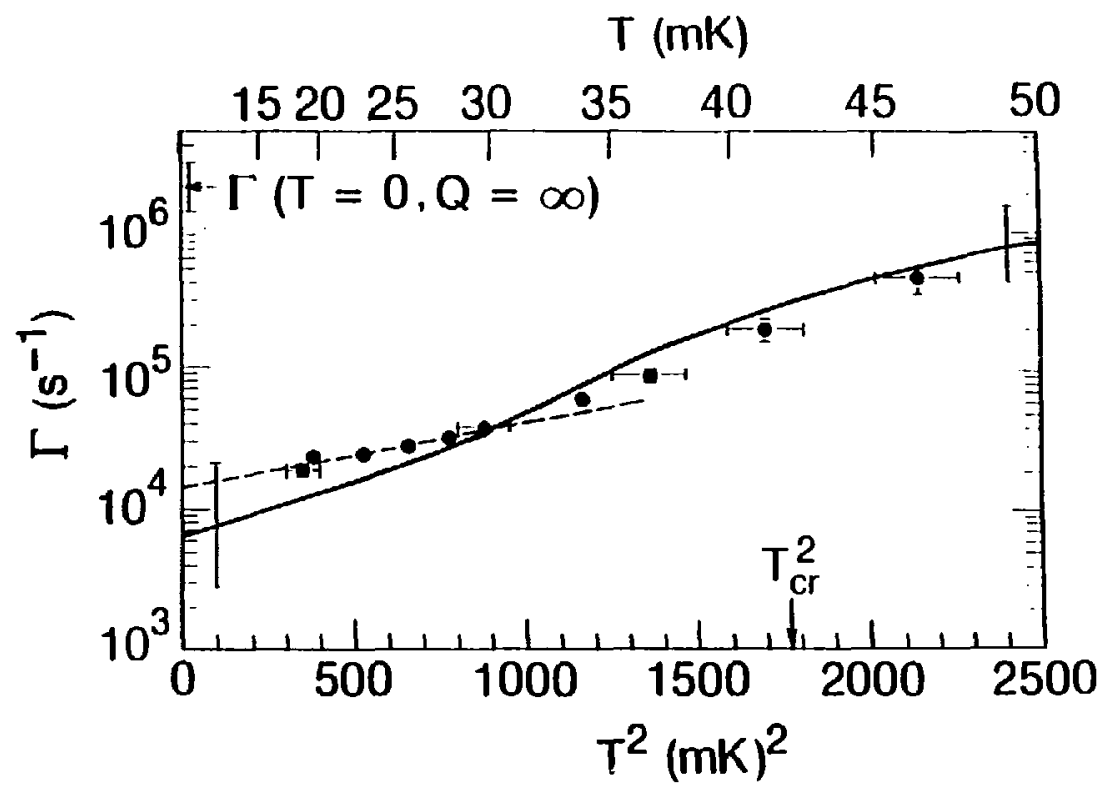

FIGURE 4.6 Low-temperature dependence of $\Gamma$ on $T^{2}$ for bias current $I=24.710 \mu A$. The dots show the experimental results, and the solid curve is the theoretical prediction, with vertical error bars from uncertainty in the junction parameters. Horizontal error bars indicate uncertainty in the temperature calibration. The dashed line is a least-squares fit to the data below $30 \mathrm{mK}$. The escape rate for the infinite- $Q$ system is indicated, as is the crossover temperature $T_{c r}$. 


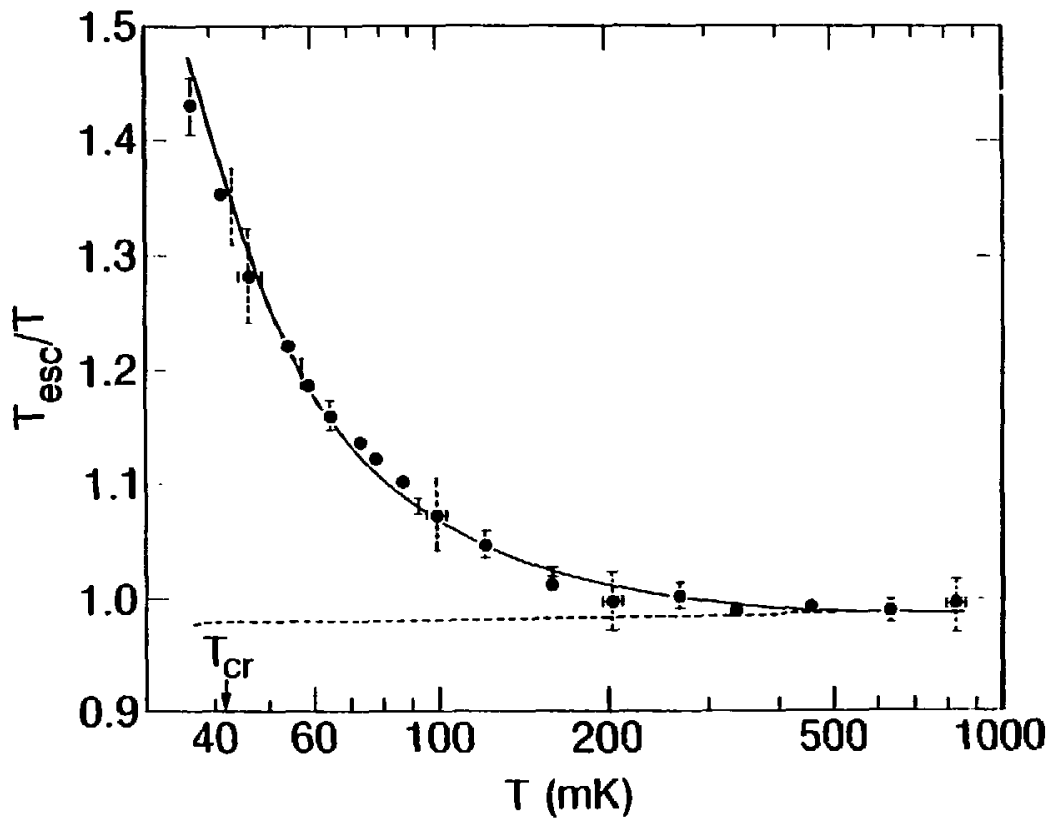

FIGURE 4.7 $\mathrm{T}_{\text {esc }} / \mathrm{T}$ vs $\mathrm{T}$. The solid curve is the theoretical prediction, and the dashed line is the classical prediction. The barrier height $\Delta U / k_{B} T$ ranges from 12.4 to 14.0 over the range of temperatures $T$. The dashed error bars indicate uncertainty in the temperature calibration, while the solid error bars indicate uncertainty in the junction parameters. 


\section{CHAPTER 5}

\section{Small Tunnel Junctions: \\ Introduction}

In this chapter I introduce the basic ddeas behind the small tunnel junction experiments, and describe the experimental design for the small junction experiments. 


\subsection{Small Tunnel Junctions - Introduction to Theory}

From the discussion of the theory of macroscopic quantum tunneling in Chapter 2, the classical equation of motion for the phase variable in a resistively-shunted Josephson junction results in a Hamiltonian for the system given by

$$
H=\frac{Q^{2}}{2 C}+\frac{I_{0} \Phi_{0}}{2 \pi}(\cos \delta-s)+H_{r e s},
$$

where $Q$ is the charge on the junction capacitance $C, I_{0}$ is the junction critical current, $\delta$ is the phase difference of the superconducting wave functions on opposite sides of the junction, $s=I / I_{0}$ is the bias tilt, and $H_{\text {res }}$ is the Hamiltonian describing the shunt resistance. The term which describes the transfer of single electrons (quasielectrons) across the junction has been left out of this expression, and I shall write this term as $\mathrm{H}_{\mathrm{T}}$. For the considerations of MQT, this term is negligible.

The variables $Q$ and $\delta$ can be treated as quantum variables, as discussed earlier. In a classical Josephson junction, the capacitance $C$ is large enough that the charging energy $\mathrm{E}_{Q}=\mathrm{e}^{2} / 2 \mathrm{C}$ is much smaller than the Josephson coupling energy $E_{J}=I_{0} \Phi_{0} / 2 \pi$, i.e. $E_{Q} \ll E_{J}$. The charging energy is also much less than the thermal energy, $E_{Q} \ll k_{B} T$. The quantum behavior of the junction can then only be seen when one attempts to localize the phase variable over a very small range, $\Delta \delta \ll 2 \pi$. In this limit, one can observe quantum tunneling of the junction from the zero-voltage state to the voltage state. In crder to make the quantum behavior of the junction more obvious, one has to increase the charging energy $E_{Q}$ with respect to $E_{J}$ and $k_{B} T$. In 
other words, one has to decrease the junction capacitance. The behavior of junctions with $E_{Q}>E_{J}$ and $E_{Q}>k_{B} T$ was investigated by several theorists (19), but the experimental situation remains unclear.

A much simpler situation results when one simply sets the critical current of the junction to zero, which is achieved experimentally by applying a magnetic field strong enough to drive the junction into the normal state. This case was invertigated by several authors (20). In this case the Hamiltonian simplifies to

$$
H=\frac{Q^{2}}{2 C}+H_{T}+H_{r e s} \text {. }
$$

The term $\mathrm{H}_{\mathrm{T}}$ giving the single electron transfer is usually written as

$$
H_{T}=\sum_{\sigma k q} M_{k q} c^{*}{ }_{k \sigma} c_{q \sigma}+H . C .
$$

where $M_{k q}$ is the transfer matrix element, $c_{k \sigma}$ is the electron destruction operator for one junction electrode, and H.C. stands for Hermitian conjugate. The sum is over both electron spins $\sigma$ and all wavevectors $k$ and $q$ in both electrodes.

If I ignore the single-electron transfer term and the resistor Hamiltonian, the Hamiltonian of Eq. (5.2) is just that of a particle with momentum proportional to $Q$, moving in free space. The energy-momentur. relation is $E=Q^{2} / 2 C$, the charging energy of a capacitor. If I now introduce the term $\mathrm{H}_{\mathrm{T}}$, assuming it is a small perturbation, I can calculate the tra' $\mathrm{isition}$ rate of the system from an initial state with charge $Q$ to a final state with charge $\mathrm{Qte}$, as these are the states coupled by $H_{T}$. A somewhat ad hoc 
approach is needed at this point; the finite capacitance of the junction causes an electron to lose energy as i: traverses the junction barrier. This energy loss is not included in the expression of Eq. (5.3); see however the discussion in Chapter 7. As an electron moves across the junction barrier, the charging energy changes by $\Delta E=E_{\text {initial }}-E_{\text {final }}=Q^{2} / 2 C-(Q \pm e)^{2 / 2} C=e( \pm 2 Q-$ e)/2C. This energy is given up by the tunneling electron. Note that I am using an electron charge of $-e$, i.e. $e$ is a positive number. If I start with $Q>0$, and assume the right side of the junction is at the higher voltage, an electron transferring from the left side of the junction, $i . e$. the side with higher electron energy, will cause $Q$ to become $Q-e$, and the electron will give up er.. gy $\Delta E=e(2 Q-e) / 2 C$. If $0<Q<e / 2$, then $\Delta E<0$, and the electron has to gain energy in traversing the barrier. At $T=0$, this is not possible, and the transfer rate is zero. If $Q>e / 2$, the electron loses energy during transfer, and the transfer rate is non-zero.

In order to estimate the total transfer rate, I use the standard semiconductor tunneling model (47). The zero-temperature current-voltage characteristic is then as sketched in Fig. 5.1. The current $I$ is related to the transfer rate $\Gamma$ by $I=\mathrm{e} \Gamma$, and the voltage to the charge by $\mathrm{V}=\mathrm{Q} / \mathrm{C}$.

The main features of the I-V of Fig. 5.1 are that there is a range of voltages $(-\mathrm{e} / 2 \mathrm{C}<\mathrm{V}<\mathrm{e} / 2 \mathrm{C})$ where no tunneling occurs, so that $\mathrm{I}=0$, and on either side of that region the current increases linearly with voltage:

$$
\begin{array}{ll}
\mathrm{I}=0 & (\mathrm{e} / 2 \mathrm{C}<\mathrm{V}<\mathrm{e} / 2 \mathrm{C}) \\
=\frac{1}{R_{\mathrm{T}}}(\mathrm{V} \pm \mathrm{e} / 2 \mathrm{C}) & (|\mathrm{V}|>\mathrm{e} / 2 \mathrm{C})
\end{array}
$$


where I have used $R_{T}$ as the junction tunneling resistance. The region of zero current is known as the "Coulomb blockade" region.

It is at this point unclear how the small capacitance tunnel junction is related to the discussion of MQT; the lack of a phase variable makes the two problems seem unrelated. However, this is now in the extreme limit of a "quantum" Josephson junction: in a classical junction, the phase $\delta$ is extremely well-defined, $\Delta \delta<2 \pi$, and the charge $Q$ uncertain, $\Delta Q \gg$ e. Here the limit is where the charge $Q$ is more precisely known, $\Delta Q \ll e$, and I can define the phase variable $\delta$ as

$$
\delta=\frac{\mathrm{e}}{\mathrm{hC}} \int \mathrm{Qdt}
$$

This definition is identical to the equation relating charge and phase in a Josephson junction, with the replacement of $2 e$ by $e$. For the normal junction, the Hamiltonian does not involve the phase, and the phase value is therefore completely uncertain. The connection between this extreme limit and that of MQT will become more clear later (see Chapter 7).

All the discussion thus far is for temperature $T=0$. At any finite temperature the Fermi distribution of each electrode will becone smeared, with the electrons having a range of energies $\Delta E \equiv k_{B} T$ about the Fermi level $\mu_{F}$. Clearly the Coulomb charging energy $E_{Q}=e^{2 / 2 C}$ must satisfy $k_{B} T$ $\ll \mathrm{E}_{\mathrm{Q}}$ for the Coulomb blockade of Fig. 5.1 to be clearly visible. With present lithographic techniques one is able to fabricate junctions with areas of order $0.01 \mu \mathrm{m}^{2}$ and corresponding capacitances of order $1 \mathrm{fF}$. The inequality $E_{Q} \gg k_{B} T$ is then satisfied for $T \ll 1 K$, implying that these small junction experiments must be performed using a dilution refrigerator. 


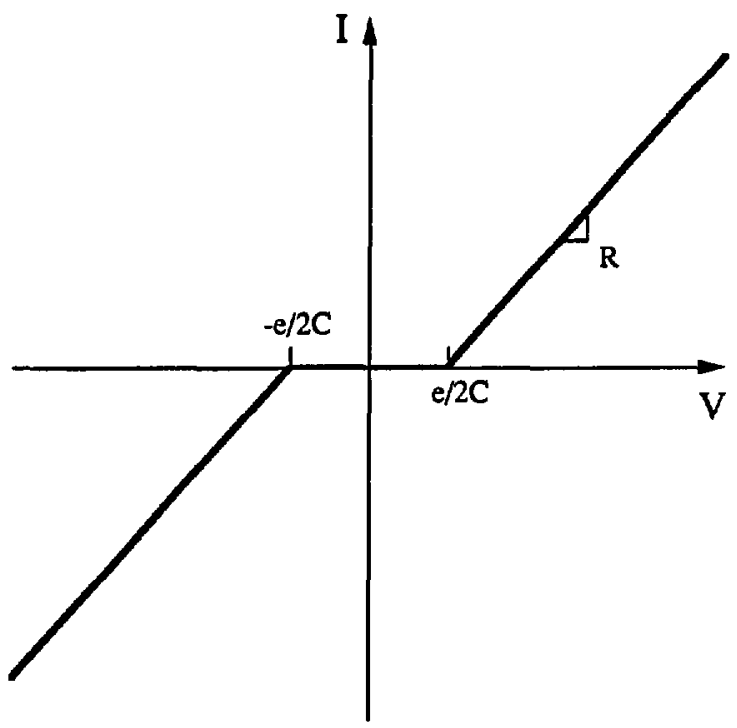

FIGURE 5.1 Current-voltage characteristic predicted from the simple small junction theory, for a voltage bias at $T=0$. The voltage region between $\mathrm{e} / 2 \mathrm{C}$ and $+\mathrm{e} / 2 \mathrm{C}$ is known as the Coulomb blockade region. 


\subsection{Design Considerations}

The simple theory cutlined in the previous section assumes that the impedance of the environment of the small tunnel junction is infinite. The charging energy only includes the capacitance $\mathrm{C}$ of the junction itself, and during the tunneling process the charge $Q$ on the junction is fixed, other than the change in $Q$ due to the tunneling electron itself. In reality, however, the leads that are attached to either junction electrode will necessarily introduce large stray capacitances, and will unavoidably present a high-frequency impedance of $10^{2}$ to $10^{3} \Omega$. As discussed in Chapter 1 , the effect of the large stray capacitance and small impedance presented by the external leads is thought to strcngly affect the small junctions. This is probably the explanation for the behavior seen in multiple and single junction experiments; see Chapter 7.

In order to minimize the effect of the stray capacitance and the low impedance leads, I designed the layout of the junctions to include thin-film resistors on the chip. In the ideal case, an infinite resistance at all relevant frequencies inserted between the small junction and the rest of the circuit will isolate the junction, as it then takes an infinite amount of time to resupply the junction with charge from the external leads: for a lumped lead resistance $R$, it takes a time $\mathrm{RC}$ to recharge the junction capacitance $\mathrm{C}$. In the absence of readily available infinite resistance leads, theoretical calculations indicate that a shunt resistor must be larger than the quantum of resistance $\mathrm{R}_{\mathrm{Q}}=$ $\mathrm{h} / 2 \mathrm{e}^{2}=6.45 \mathrm{k} \Omega$ for the electrons to be well-localized on either side of the junction (20). The leads must therefore have at least $6.45 \mathrm{k} \Omega$ of resistance.

The tirst design attempt for the resistive leads used the same CuAu alloy as was used for the shunt resistor in the MQT experiment, with a 
resistivity $\rho$ of $12 \mu \Omega-\mathrm{cm}$. In order to get a DC resistance of about $10 \mathrm{R}_{\mathrm{Q}}$, a meander pattern was designed, where considerations of the capacitive coupling between each leg of the meander implied that the resistance wuld only begin to short out above about $10^{11} \mathrm{~Hz}$, sufficiently higher than the RC junction recharging time of about $10^{10} \mathrm{~Hz}$. It was only later that I recognized the importance of the self-capacitance of each lead, i.e. the capacitance to ground at infinity, and how it would act to short out the resistor at a much lower frequency than the much smaller inter-lead capacitance. A discussion of the question of self-capacitance, and how to calculate the capacitance for the types of structures described here, is given in Appendix A. Following this realization, I developed a new lead layout which consisted of straight lines for each lead (see Chapter 6), and with the advice of John Martinis, switched to a $\mathrm{NiCr}$ alloy, with a resistivity of about $120 \mu \Omega-\mathrm{cm}$.

The impedance the straight line leads present to the junction can be modeled fairly easily. A piece of metal has a self-capacitance $C_{L}$, a selfinductance $\mathrm{L}_{\mathrm{L}}$ and a resistance $\mathrm{R}_{\mathrm{L}}$, all of which carı be calculated from geometric considerations. In general, the resistance $R_{L}$ is

$$
R_{\mathrm{L}}=\rho \boldsymbol{N A}
$$

where $\Lambda$ is the lead length and $A$ the cross-sectional area. The selfcapacitance $\mathrm{C}_{\mathrm{L}}$ for an $\mathrm{SiO}_{2}$ substrate is approximately given by (see Appendix A)

$$
C_{L}=(9.8 \mathrm{fF} / \mathrm{mm}) \wedge
$$




$$
\mathrm{L}_{\mathrm{L}}=(1 \mathrm{nH} / \mathrm{mm}) \Lambda
$$

These distributed elements form a resistive transmission line, and the resulting frequency dependent impedance can be calculated (see Appendix B). The basic result is that the low-frequency impedance is $Z=R_{L}$, for frequencies $\omega<1 / R_{L} C_{L}$. The high-frequency impedance is $Z=\sqrt{R_{L} \omega C_{L}}(1-i)$ for frequencies $\omega>1 / R_{L} C_{L}$. The capacitance $C_{L}$ is fixed for a given lead length $\Lambda$, and the high-frequency impedance scales as $\sqrt{R_{L}}$. One would therefore want to maximize the lead resistance, which is done by using a high resistivity material and by using as small a crosssectional area as possible.

The difficulty one encounters when reducing the cross-section of the leads is that the hot-electron effects become very important. As discussed earlier (see Chapter 4), the electrons in the resistor will lose thermal contact with the phonons, and the dissipation of power $P$ in the resistive leads will heat the electrons to a temperature $T_{c}$ above the phonon temperature $T_{p h}$, given by

$$
\frac{P}{A \Lambda}=\left(2 \times 10^{9} \mathrm{~W} / \mathrm{m}^{3} \mathrm{~K}^{5}\right)\left(\mathrm{T}_{\mathrm{e}}^{5}-\mathrm{T}_{\mathrm{ph}}^{5}\right)
$$

where $A$ is the cross-sectional area and $\Lambda$ the length of the resistor. Given a power dissipation $P=\rfloor^{2} R_{L}$ for a current $I, I$ find that

$$
T_{e}^{5}=T_{p h^{5}}+\left(5 \times 10 \cdot 10 m^{3} K^{5} / W\right) I^{2} p / A^{2}
$$


This result is plotted in Fig. $\mathbf{5 . 2}$ as a function of bias current I, for both the $\mathrm{CuAu}$ and $\mathrm{NiCr}$ materials in different geometries. Clearly the resistivity should be minimized and the cross-sectional area $\mathrm{A}$ maximized to reduce the electron heating, in direct conflict with the need for high impedances at high frequencies. The design I used, with a $30 \mathrm{~nm}$ thickness and a $2 \mu \mathrm{m}$ linewidth, for both the $\mathrm{CuAu}$ and $\mathrm{NiCr}$ materials, will show heating for currents in excess of about $1 \mathrm{nA}$. If I consider just the first $4.5 \mathrm{~mm}$ of each lead (the straight segment leading out from the junction), the leads will look like resistors for frequencies $\omega$ up to about $2.5 \mathrm{GHz}$ for the CuAu alloy and about $170 \mathrm{MHz}$ for the NiCr. The CuAu will have shorted out to the superconducting quantum of resistance of $6.45 \mathrm{k} \Omega$ at a frequency of about $4.4 \mathrm{GHz}$, and the $\mathrm{NiCr}$ at a frequency of about $30 \mathrm{GHz}$. 


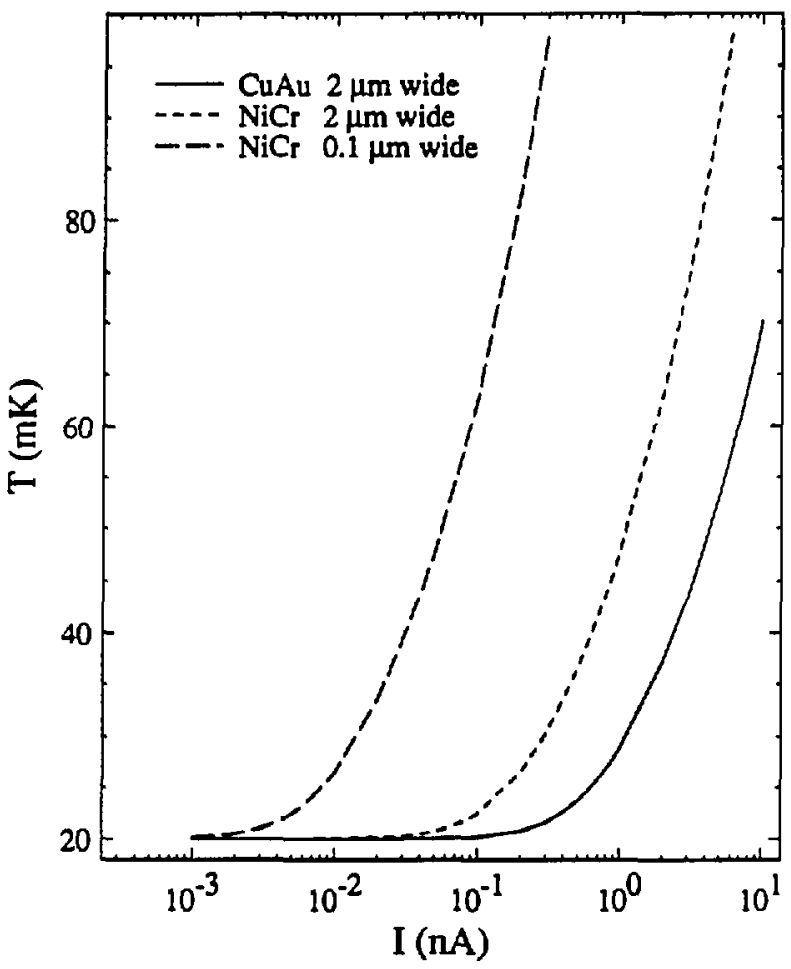

FIGURE 5.2 Calculated dependence of resistor temperature T on DC bias current I, for different resistive lead configurations and materials. The calculations assume a phonon temperature $T_{p h}=20 \mathrm{mK}$. 


\title{
CHAPTER 6
}

\author{
Small Tunnel Junctions: \\ Fabrication and Sample Mount
}

In this chapter I describe the method of small junction and resistor fabrication, as well as the junction mount and wiring on the dilution refrigerator. 


\subsection{Sample Fabrication}

Fabrication of the samples used in this experiment involved a combination of optical and scanning electron microscope (SEM) lithography. Optical lithography was done in the Microfabrication Laboratory in Cory Hall, and the SEM exposures were performed using a ETEC Autoscan SEM, located in 144B Cory Hall.

All the samples were made on $\mathrm{Si}$ wafers $5.08 \mathrm{~cm}$ in diameter, either $<100>$ or $<111>$ orientation, $0.35-0.5 \mathrm{~mm}$ thick, with resistivities of order 1-10 $\Omega-\mathrm{cm}$. Each wafer had a $1000 \mathrm{~nm}$ thick $\mathrm{SiO}_{2}$ insulating layer grown on one face; this face was used as the substrate for the series of metal evaporations which defined the tunnel junction and the leads attached to it.

I present a standard recipe for the optical lithography which was used to pattern the leads and contact pads, and a recipe for the SEM lithography which was used to pattem the tunnel junctions.

\section{Optical Lithography}

All the patteming on the wafers was done using liftoff of evaporated metal layers which overlay a partemed photoresist layer. I used one fixed recipe for the photoresist patterning, which is the recipe described in Chapter 3.

\section{SEM Lithography}

The SEM lithography would only have been possible with the use of the ETEC Autoscan, for which hardware and software for lithographic applications had previously been developed. I am most grateful to David Chin and Prof. T. van Duzer of the EECS Department for allowing me to use 
this SEM, and to David Chin and Dan Courtney of Perkin-Elmer for helping out when the SEM had technical problems. I would also like to thank Tom Holm of KTI Chemicals for donating the resists I used for lithography.

The recipe I give here has not been carefully optimized. The minimum standard linewidth I was able to achieve was about $100-150 \mathrm{~nm}$; the smallest linewidth I observed was $60 \mathrm{~nm}$. It is conceivable that a careful optimization of the recipe parameters could reduce this by a factor of 2 or more. All processing at this point was done on single $11 \mathrm{~mm} \times 11 \mathrm{~mm}$ chips, as that was the largest sample that could be fit into the SEM chamber. The recipe was adapted from published methods $(49,50)$

1. Spin on the bottom layer of resist, which consisted of 9 wt.\% poly(methylmethacrylate-methacrylic acid) dissolved in 2-ethoxy-ethanol (more easily referred to as 9\% P(MMA-MAA) in Cellosolve). A spin speed of $3000 \mathrm{rpm}$ for 30 seconds gave a baked film thickness of $400 \mathrm{~nm}$.

2. Bake the chips in a $150^{\circ} \mathrm{C}$ oven for 1 hour.

3. Spin on the top layer of resist, which was $2 \mathrm{wt} . \%$ poly (methylmethacrylate) (PMMA) in chlorobenzene. A spin speed of 3000 rpm gave a baked film thickness of $100 \mathrm{~nm}$.

4. Bake a second time at $150^{\circ} \mathrm{C}$ for 1 hour.

5. Expose the chips on the SEM (see below).

6. Develop the chips in a Cellosolve-methanol mixture for 6 seconds, and then rinse the chip in methanol. Blow dry with compressed gas. The best mixture ratio appeared to be 1 part Cellosolve (also called 2-ethoxy-ethanol) to 2 pars methanol.

The linewidh was fairly sensitive to the exposure parameters: The chip was placed about $8 \mathrm{~mm}$ below the final aperture (the z-axis vernier was set at $6.6 \mathrm{~mm}$ ). I used a magnification of $\times 400$, an accelerating voltage of 20 
$\mathrm{kV}$, and a condenser current of at least 3.0 A. The condenser current was set so that the beam current hitting the Faraday cup on the chip mount was 1.0 pA. I believe the collection efficiency of this cup was $10-20 \%$, so the actual beam current was probably 5-10 pA. The SEM was carefully focussed on the surface of the resist just before exposure; this was done by putting a drop of methanol, which had submicron-sized $\mathrm{Ag}$ particles suspended in it, on the chip surface, and focussing carefully on the $\mathrm{Ag}$ particles left behind after the methanol evaporated.

The LSI-1 1 (from Digital Equipment Corp.) that controlled the SEM divided the $x$ - and $y$-axes into 4096 grid points, each axis numbered from 0 to 4095 with $(0,0)$ in the lower left comer. Lines were drawn with the SEM by directing the beam to hit a series of chosen grid points for a fixed length of time (a version of Missile Command!). The best exposures I was able to get were achieved by drawing two rectangles, one with its upper left comer at $(x, y)=(0,2000)$ and lower right at $(2000,1999)$, and the other with upper left at $(2014,2000)$, lower right at $(4096,1999)$. Each rectangle consisted of two adjacent rows of points, where on each row svery other point was exposed, and the optimal exposure time was usually 2.5-3.0 ms. In terms of the parameters used by the LSI-11 software, I was using the values given in Table 6.1. In addition to these lines, I would draw large square rectangles at the extreme right and left to make contact between the Al that made up the junction and the leads.

The developed pattern consisted of the two rectangles, as drawn, appearing in the top resist layer, separated from one another by a bridge about $200 \mathrm{~nm}$ in width, and a larger rectangle in the more sensitive lower resist layer, about 600-700 $\mathrm{nm}$ wide, which undercut the bridge (see Fig. 6.1). 


\begin{tabular}{cccccccc}
\hline Rectangle & (xs,ys) & (xe,ye) & d & t & n & f & dir. \\
\hline 1 & $(0,2000)$ & $(2000,2000)$ & 30 & 2 & 2 & 1 & right \\
2 & $(4096,2000)$ & $(2014,2000)$ & 30 & 2 & 2 & 1 & left \\
\hline
\end{tabular}

TABLE 6.1 Parameters for the SEM exposures which defined the small junctions. The coordinates ( $x s, y s)$ define the start point, (xe,ye) the end point, $d$ the exposure dose (number of time intervals to expose a point), $t$ the time unit ( 2 means $10^{-4}$ sec.), $n$ the number of points to jump along a line ( 2 means expose every other point), and $f$ the number of lines to skip in an exposure frame ( 1 means draw on every line). Dir is the direction to tum at the end of a line. 


\section{Evaporations}

Metal evaporations consisted of evaporations of $\mathrm{Au}, \mathrm{CuAu}$ alloy, $\mathrm{NiCr}$ alloy, and $\mathrm{Al}$.

Au evaporations were done by placing a length of 0.99 purity Au wire in a W boat, and evaporating $30-40 \mathrm{~nm}$ of $\mathrm{Au}$ at a rate of about $0.1 \mathrm{~nm} / \mathrm{sec}$, as measured by a quartz crystal monitor. A $\mathrm{Cr}$ underlayer 2-3 nm thick was evaporated first to ensure adhesion of the Au layer.

CuAu alloy (25wt.\% Cu), the material used for the MQT shunt resistors, was evaporated to a thickness of $30 \mathrm{~nm}$, as described in Chapter 4 . As for the Au films, a $\mathrm{Cr}$ underlayer 2-3 nm thick was evaporated before the CuAu alloy. These films had a resistance of about $4 \Omega$ per square, or a resistivity of about $12 \mu \Omega-\mathrm{cm}$.

The $\mathrm{NiCr}$ films were made by wrapping a length of $\mathrm{NiCr}$ wire around a 3-stranded-wire $\mathrm{W}$ basket. The wire evaporated where it touched the basket, and the basket had to glow white-hot to get a reasonable evaporation

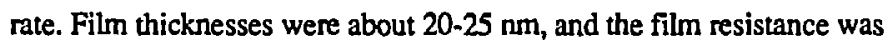
about $60 \Omega$ per square, corresponding to a resistivity of $120 \mu \Omega-\mathrm{cm}$.

$\mathrm{Al}$ evaporations were done by placing lengths of 0.9999 purity $\mathrm{Al}$ wire in two 3-stranded-wire W baskets. A W boat was also filled with 0.99999 purity $\mathrm{Cu}$ shot. A Cu layer about $5 \mathrm{~nm}$ thick was evaporated first to ensure good electrical contact between the $\mathrm{Al}$ and the $\mathrm{Au}$ contact pads at the end of the resistive leads. Without this layer the $\mathrm{Al}$-Au contacts tended to increase in resistance over a period of a few hours. The evaporation was done at an angle that ensured that the evaporated $C u$ would not be anywhere near the junction area itself. The first Al layer was evaporated with a film thicknesses of about $40 \mathrm{~nm}$, with a substrate angle of about $30^{\circ}$ to the boat, and with a somewhat high evaporation rate of about $10 \mathrm{~nm} / \mathrm{sec}$. This rate 
a)

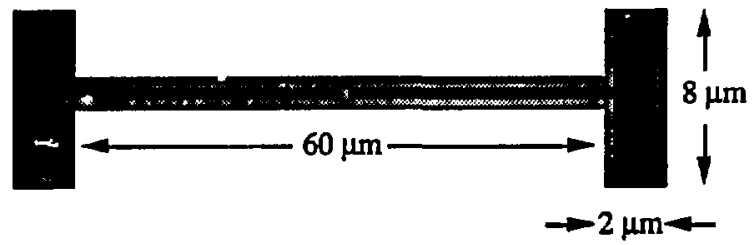

Patten in bottom resist layer

Pattem in top resist layer

b)

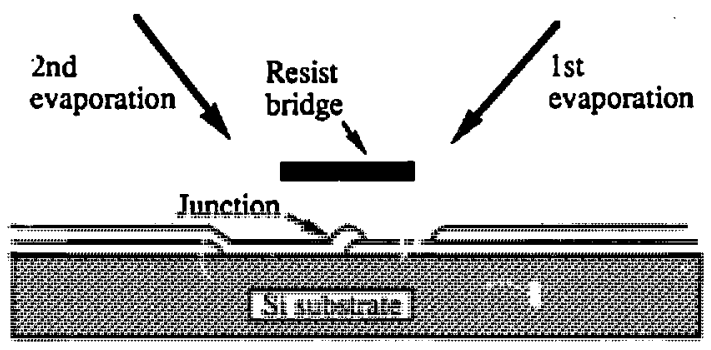

FIGURE 6.1 (a) Pattem developed out in the top (PMMA) and bottom (P(MMA-MAA)) electron beam resists for the shadowed junction evaporations. The bottom layer is more sensitive and therefore develops out further than the top layer. (b) Schematic of the angled evaporation technique used to form the junction. 
was used to prevent the $\mathrm{Al}$ from oxidizing before it reached the substrate. This layer of $\mathrm{Al}$ was oxidized in about $\mathrm{C} \leq$ Torr of $30 \% \mathrm{O}_{2} / \mathrm{Ar}$ for 10 minutes. The substrate was then tilted in the opposite direction to make an angle of about $30^{\circ}$ to the second $\mathrm{W}$ basket, and another 40 nm thick Al film was evaporated on the substrate. The Al was lifted off in boiling acetone. The junction was formed from the overlap of the $\mathrm{Al}$ rectangles from the two evaporations; the precise angles determined the area of the junction (see Fig. 6.1). The typical junction areas were about $0.02 \mu \mathrm{m}^{2}$, and the contacts to the Au pads at the other end of the Al leads were about $10 \mu \mathrm{m}^{2}$ in area.

\section{Sample Design}

The central idea in the design of the resistive leads was to reduce stray capacitance and to maximize the impedance of the leads to as high a frequency as possible (ideally up to at least $10^{10} \mathrm{~Hz}$ ). In the first chip design, it was recognized that the DC lead resistance had to be at least of order 100 $k \Omega$ (so that $R_{L}=10 R_{Q}$ ), but the relatively low resistivity of the CuAu alloy forced a design which included a large number of meanders on each lead to reach this value. Each meander would capacitively couple across itself, so the spacing and length of each meander was chosen so that this coupling would begin to roll the impedance off only above $10^{10} \mathrm{~Hz}$. The selfcapacitance of the leads was ignored. Thus the first design involved two $2 \mu \mathrm{m}$ wide lines cuming straight out of each end of the Al leads to the junction, extending $4.5 \mathrm{~mm}$ to near the edge of the chip, and then moving in a $2 \mu \mathrm{m}$ linewidth meander around the perimeter of the chip to the four $0.5 \mathrm{~mm} \times 0.5$ $\mathrm{mm}$ contact pads (see Fig. 6.2).

It was liter discovered that the self-capacitance of the $2 \mu \mathrm{m}$ leads was sufficient to effectively short out all but the first few millimeters of the leads 
above about $10^{8} \mathrm{~Hz}$ (see Appendix A). The meanders were therefore not providing any significant resistance above that frequency, and thus could be eliminated. Furthermore, I developed techniques for making higher resistivity alloys which obviated the need for the meanders. Thus in the second chip design the meanders were replaced by straight lines to the contact pads (see Fig 6.3), which greatly reduced the horror of having to make unbroken $2 \mu \mathrm{m}$ wide lines $120 \mathrm{~cm}$ in length.

The large contact pads on the edges of the chip were made of $40 \mathrm{~nm}$ thick Au films. Adjacent pads were connected to each other by a $100 \mu \mathrm{m}$ wide Au strip, which protected the junctions from static charge buildup. Small $4 \mu \mathrm{m} \times 6 \mu \mathrm{m}$ Au contact pads were also placed at the end of each lead closest to the junction. These pads provided a consistent contact surface for the Al.

The $\mathrm{Al}$ junctions themselves were relatively simple. The SEM pattern and evaporation technique are described above. Contacts to each of the four $4 \mu \mathrm{m} \times 6 \mu \mathrm{m}$ Au pads provided a nearly four-point measurement (only nearly because two $30 \mu \mathrm{m}$-long Al leads led from the contact pads to the junction itself; see Fig. 6.3). 


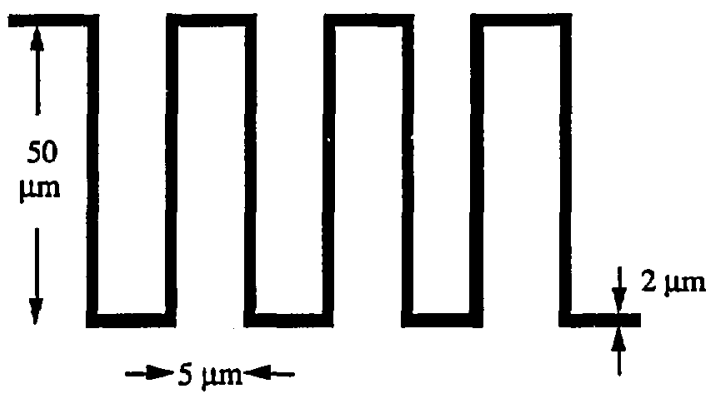

FIGURE 6.2 Layout of meander pattem for CuAu alloy resistors. The meander linewidth, spacing and length are indicated in the figure. Each of the resistive leads included such meanders, after a $4.5 \mathrm{~mm}$-long straight segment leading out from the junction. The total length of each lead was 120 mm. 


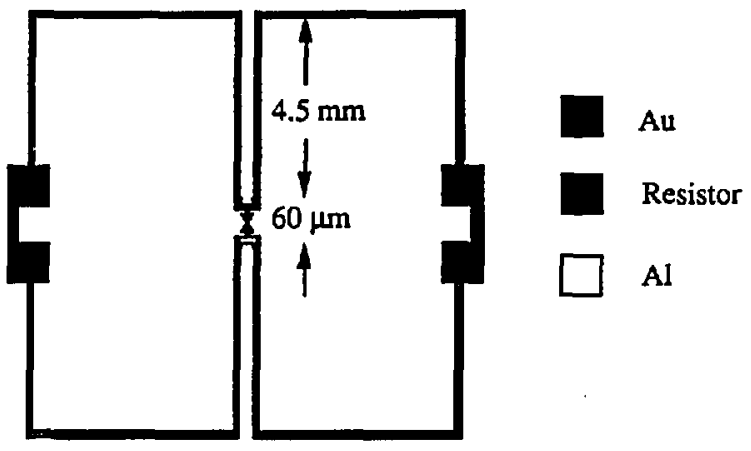

FIGURE 6.3 Full layout for the small junction measurements, used for the $\mathrm{NiCr}$ leads. The $\mathrm{CuAu}$ leads had meanders after the first $4.5 \mathrm{~mm}$ leading out from the junction. The connections between the leads at the chip edge are $\mathrm{Au}$ shoring strips that were scribed through after the junction was mounted. 


\subsection{Single Tunnel Junction Mount}

T.ue mount used for the small junction experiments was designed with two aims: one was reduce the capacitive coupling between the resistive leads and any metal parts of the mount, and the other was to enable us to drive the Al junctions into the normal state.

The mount I used for most of the measurements (two other mounts were made and discarded for different reasons) consisted of a $\mathrm{Cu}$ rod which screwed onto the mixing chamber of the dilution refrigerator. This rod had a face with screw holes to mount the $\mathrm{Ge}$ and carbon resistive thermometers, and another face to which a second $\mathrm{Cu}$ piece was bolted. This second $\mathrm{Cu}$ piece had a piece of G-10 fiberglass glued to it with Stycast 2850, and a glass slide was in turn glued to the fiberglass with the same epoxy. The junction rested on the glass slide and was at least $8 \mathrm{~mm}$ from any metal surfaces. The four current and voltage leads were attached to the junction chip with In blobs. Each lead passed in a tightly wound spiral through a block of 300 mesh stainless steel powder mixed with Stycast 2850 epoxy. This structure formed a microwave filter with at least $10 \mathrm{~dB}$ of attenuation above $1 \mathrm{GHz}$ (see the discussion of the Cu powder filters in Chapter 3). The leads, junction and steel powder block were encased in a loosely fitting $\mathrm{Cu}$ box made of thin $\mathrm{Cu}$ sheet, meant to act as a radiation shield and Faraday cage. The two current and two voltage leads then went up the refrigerator as two twisted pairs of 75 $\mu \mathrm{m}$ diameter manganin wire inside a rigid CuNi tube.

The Al junctions were driven into the normal state by using two $\mathrm{Nd}$ Fe-B permanent magnets, $1 / 8$ " thick, one of which was $1 / 2$ " in diameter, the other $1 / 4$ " in diameter; these were purchased from the Edmund Scientific Co. These magnets faced the chip surface from above and below, each $8 \mathrm{~mm}$ 
from the chip surface, and a cold-rolled steel yoke was placed around them to increase the field strength at the junction (cold-rolled steel has a large magnetic permeability). A sketch is shown in Fig. 6.4. Measurements with a Hall probe gave a field strength of 1100 Oe at the chip surface; bulk Al has a critical field of about $100 \mathrm{Oe}$, but the small dimensions of the junction electrodes increase the critical field to about $400-500$ Oe (see e.g. van Duzer and Tumer (51)). 


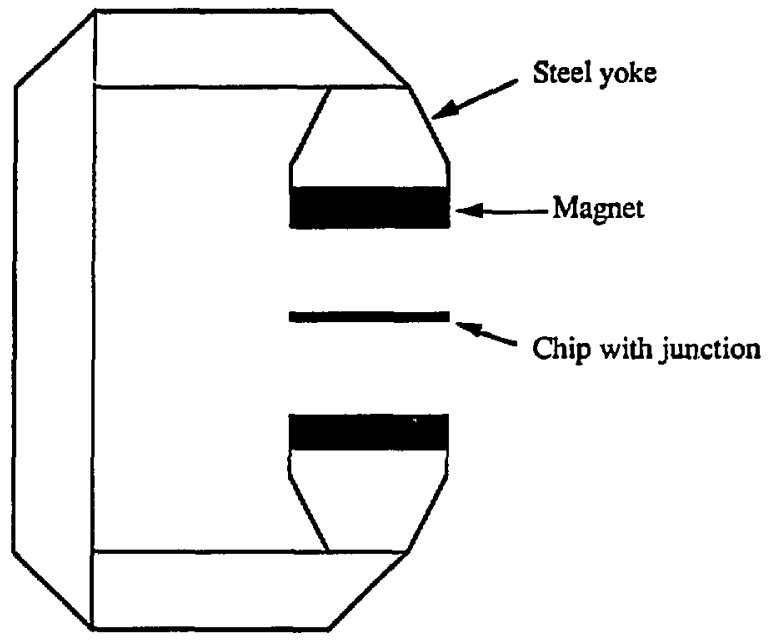

FIGURE 6.4. Cold-solled steel yoke with permanent Nd-Fe-B magnets used to drive the $\mathrm{Al}$ junction into the normal state. This drawing is two times actual size. 


\subsection{Dilution Refrigerator}

The dilution refrigerator I used for the small tunnel junction measurements was the same as the one used for the MQT experiments (see the description of the refrigerator and the thermometry in Chapter 3). However, the wiring on the refrigerator was changed to accommodate a single four-point measurement on the small junctions, as opposed to using the coaxial two-point measurement on the MQT junction.

The wiring on the refrigerator for the small tunnel junction measurements consisted of two twisted pairs, one pair for the current bias and the other pair for voltage measurements, and a single coaxial lead for injection of mdiofrequency signals. In blobs were used to attach the four current and voltage leads to the sample. Each lead then passed through a steel powder-epoxy block (see section 6.2), which was attached to the sample mount. The four wires were then twisted into pairs, each pair running through a $1 / 16$ " diameter CuNi tube. These tubes were thermally grounded at the mixing chamber, the plate and the still, before passing through feedthroughs in the vacuum can flange into the $4 \mathrm{~K}{ }^{4} \mathrm{He}$ bath. The four wires then passed through radiofrequency RC filters potted in stainless steel powder and epoxy, with a $3 \mathrm{~dB}$ rolloff point at $16 \mathrm{kHz}$. These filters were immersed in the ${ }^{4} \mathrm{He}$ bath. Upon leaving the ${ }^{4} \mathrm{He}$ dewar, each lead passed through a room-temperature EMI radiofrequency filter, with $120 \mathrm{~dB}$ attenuation above $100 \mathrm{MHz}$. A schematic is shown in Fig. 6.5.

The radiofrequency coaxial line used for injecting microwave signals included three attenuators, a $6 \mathrm{~dB}$ attenuator just inside the ${ }^{4} \mathrm{He}$ dewar flange, a $6 \mathrm{~dB}$ atrenuator just above the vacuum flange (in liquid ${ }^{4} \mathrm{He}$ ), and a $20 \mathrm{~dB}$ attenuator bolted to the still. These attenuators, made by Narda, were $50 \Omega$ 
flat frequency response filters. This line was terminated by a weak capacitive coupling to the $\mathrm{I}^{+}$current lead on the sample mount.

The dilution refrigerator was enclosed in a Cu-mesh screen room.

The sample therefore was surrounded by five successive shields: the screened room, the ${ }^{4} \mathrm{He}$ dewar (which was made of $\mathrm{Al}$ ), the vacuum can (stainless steel), the still heat shield (Cu) and the $\mathrm{Cu}$ sheet box surrounding the sample mount. There was also a $\mu$-metal shield around the ${ }^{4} \mathrm{He}$ dewar, with the option of adding a second $\mu$-metal shield. 


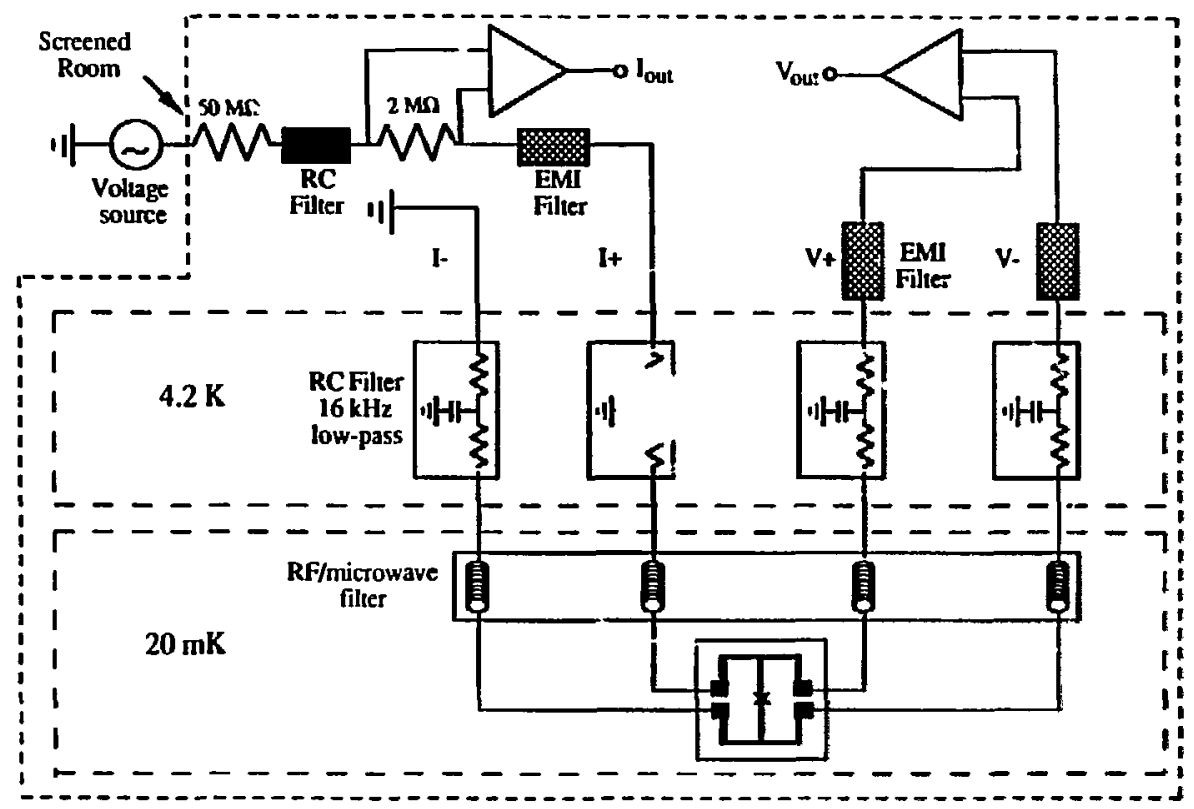

FIGURE 6.5 Wiring schematic for dilution refrigerator. The four-point wiring scheme includes filters at three temperature stages for each lead. 


\subsection{Sample Measurement}

All of the measurements reported here consisted of low-frequency current-voltage measurements and differential resistance measurements, and were made while the sample was mounted on the dilution refrigerator.

The sample measurements were carried out using the wiring schematic shown in Fig. 6.5. Typically a voltage source (either a mercury battery or a furction generator) was connected in series with the junction leads, a $50 \mathrm{M} \Omega$ current limiting resistor, and a $2 \mathrm{M} \Omega$ resistor across which a PAR 113 amplifier 'vas connected for measuring current. The current was also passed through a low-pass $\mathrm{RC}$ filier, with a rolloff frequency set between 3 and $100 \mathrm{~Hz}$, before going through the set of filters built into the cables on the refrigerator (see the previous section). A second PAR 113 amplifier was connected across the voltage leads for voltage measurement. Both amplifiers had about $15 \mathrm{nV} / \sqrt{\mathrm{Hz}}$ intrinsic noise, and were set with a high-frequency rolloff of $3 \mathrm{~Hz}$ to eliminate $60 \mathrm{~Hz}$ and viorational noise. Current-voltaje traces were recorded on a X-Y plotter. Both amplifiers were batterypowered, and the amplifiers, current-limiting resistors and filters were all placed inside a screaned room with the dilution refrigerator. No line power was brought into the sereen room when I performed measurements, and the line power for the $X-Y$ recorder and the function generators was passed through a Filtron radiofrequency line filter.

The derivative measurements, which were usually I vs. dV/dl tracts, were performed by using a very low frequency sweep for the current (typically I to $3 \mathrm{mHz}$ ), and a low-frequency jitter current for the lock-in measurement (typically 15 to $20 \mathrm{~Hz}$ ). Both signals were generated by function generators, as a hand-driven, battery-powered sweep proved to be 
too uneven. The jitter curnent was typically about $1 \%$ of the full amplitude of the sweep current; for a $1 \mathrm{nA}$ sweep, the jitter was about $10 \mathrm{pA}$ peak-topeak. The jitter was filtered out by the current-amplifying PAR 113, and the voltage amplifier was set with a high-frequency rolloff of $30 \mathrm{~Hz}$. The voltage amplifier output was subsequently amplified and mixed down by a lock-in amplifier. Traces were again recorded on an X-Y plotter, and comparison with a current-voltage trace would be used to calibrate the differential resistance scale.

The two largest sources of spurious noise were due to $60 \mathrm{lz}$ pickup and vibrational noise, presumably from wires moving relative to ground. The $60 \mathrm{~Hz}$ pickup was reduced to about $20 \mathrm{nV}$ peak-to-peak across each pair of leads by very carefully isolating the screened room from all grounds (expecially those through the gas handling system), except for the one ground through the measurement system. The BNC coaxial cables used for sample measurement were passed through the screen room with isolated-ground feedthroughs, and were only grounded at the one point where the current and voltage leads left the $4 \mathrm{He}$ dewar groundplane, which was common with the screen room ground. A $\mu$-metal shield was placed around the lower part of the refrigerator at the height of the sample. A second $\mu$-metal shield could either be placed concentric to the first shield, or placed around a platform which held the amplifiers and the current limiting resistors. This platform formed a small magnetic and electric screen room, intemal to the $\mathrm{Cu}$ mesh screen room.

Vibrational noise was a little more difficult to treat, as it varied from nin to run. Generally the leads in the refrigerator were made stiff, and their motion restricted as much as possible. Some noise was picked up from screen-room motion, so I was careful not to disturb the frame of the screen 
room when performing measurements. In general there were about 10 or 15 reaks of vibrational noise in the range $1 \mathrm{~Hz}$ to $1 \mathrm{kHz}$, with the largest peaks about 10-20 nV peak-to-peak. 


\section{CHAPTER 7}

Small Tunnel Junctions:

Results and Disc ussion

In this chapter I present the results of the measurements on the small tunnel junctions. I then describe a theory involving the quantum Langevin equation that gives a somewhat phenomenological explanation for the data. Following that I present the more rigorous phase correlation theory, which appeared after this work was first reported, and discuss its application to the experimental results. 


\subsection{Experimental Results}

A number of small junctions were fabricated and measured on the dilution refrigerator; a summary is presented in Table 7.1. Measurements at $20 \mathrm{mK}$ of the current-voltage characteristics of two typical junctions, one with the CuAu leads (junction 5 in Table 7.1) and the other with the $\mathrm{NiCr}$ leads (junction 7 in Table 7.1), are shown in Fig. 7.1. The dotted lines show the expected zero-temperature current-voltage characteristic. It is clear that the Coulomb blockade is only barely visible in junction 5 , while it is quite sharply defined in junction 7. Fig. 7.2 shows the measured differential resistance traces. It is clear that one must apply about $100 \mathrm{nA}$ of bias current to see the complete Coulomb blockade in junction 5 , while for junction 7 one only needs to apply about $1 \mathrm{nA}$. The increased lead resistance for junction 7 has clearly had a strong effect on the I-V characteristic, and proves the important effect the extemal impedance has on the behavicr of these small junctions.

In Fig. 7.3 I show the measured zero-bias resistance (ZBR) of a number of different junctions, as a function of the refrigerator temperature. The junctions with $\mathrm{NiCr}$ leads, shown by the solid symbols, all show a significantly higher resistance than the junctions with CuAu leads, shown by the open symbols. It is also clearly seen that the ZBR flattens out as the temperature is reduced, at a somewhat lower temperature for the junctions with the $\mathrm{NiCr}$ leads than for the junctions with CuAu leads.

A number of attempts were made to explain the behavior of the ZBR as the temperature is lowered. The possibility that the flattening is due to spurious high-frequency noise (radio- or microwave-frequency noise) was 

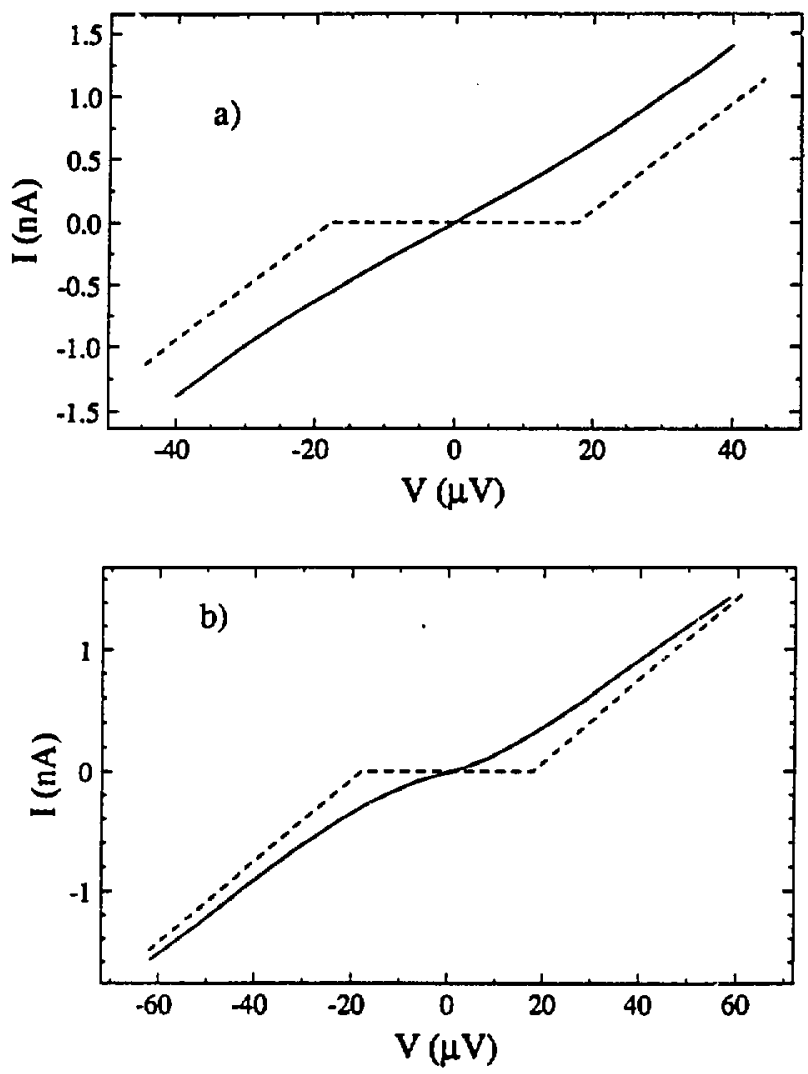

FIGURE 7.1 Current-voltage characteristics (solid lines) measured for two small junctions, at $\mathrm{T}=20 \mathrm{mK}$. (a) Junction 5 with CuAu leads, with junction resistance $R_{J}=23 \mathrm{k} \Omega$ and capacitance $C_{j}=4 \pm 1 \mathrm{fF}$. (b) Junction 7 with $\mathrm{NiC}$ leads with junction resistance $R_{J}=29.4 \mathrm{k} \Omega$ and $\mathrm{C}_{\mathrm{J}}=5 \pm 1 \mathrm{~F}$. The dotted lines show the predicted voltage-biased Coulomb blockade for each junction. 

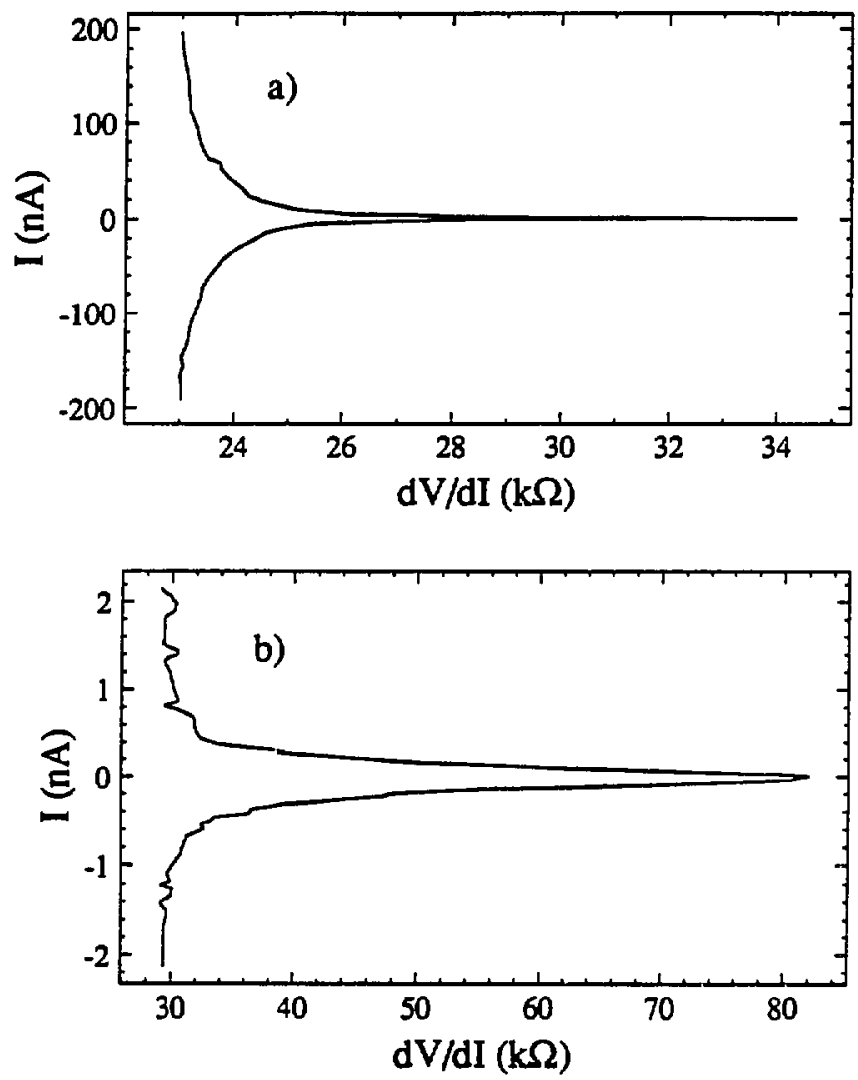

FIGURE 7.2 Measured differential resistance traces for (a) junction 5 and (b) junction 7. The traces are taken at a refrigerator temperature of $\mathrm{T}=\mathbf{2 0}$ mK; note the different current scales for (a) and (b). 


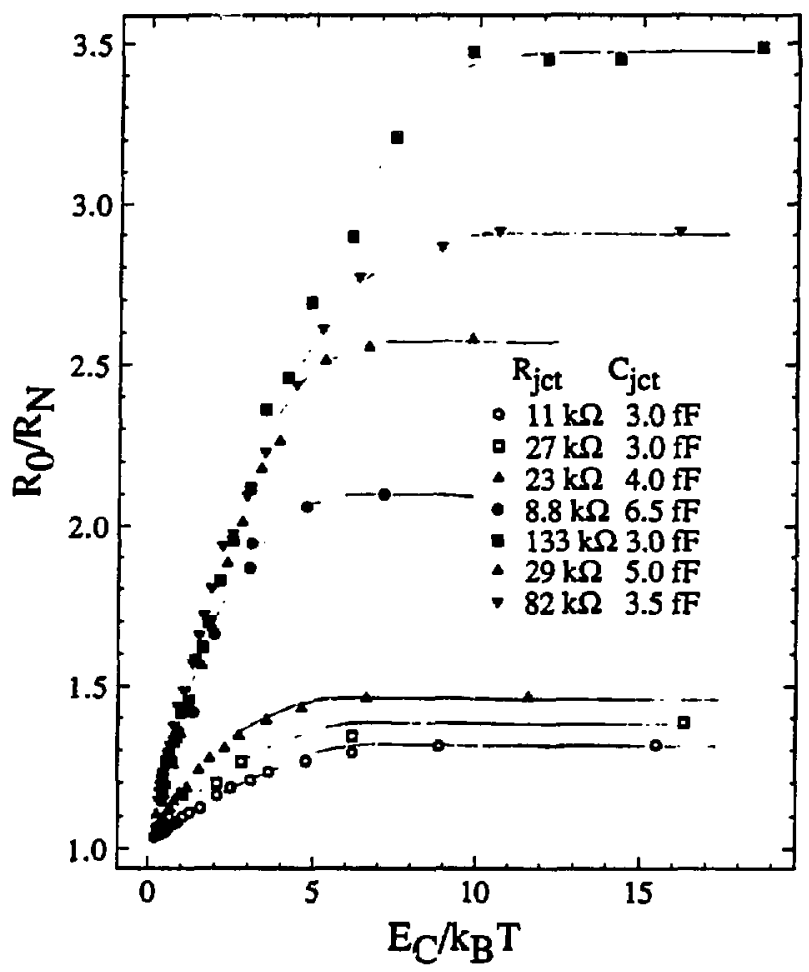

FIGURE 7.3 Zero-bias resistance as a function of temperature, as measured for a number of small junctions. The open symbols are for junctions with CuAu leads and the closed symbols for those with $\mathrm{NiCr}$ leads; the legend lists the junction resistance and capacitance, as measured from the curnent-voltage characteristic. The lines are guides for the eye. 


\begin{tabular}{cccccc}
\hline Junction & $\begin{array}{c}\mathbf{R}_{\mathbf{I}} \\
(\mathrm{k} \Omega)\end{array}$ & $\begin{array}{c}\mathrm{C}_{\mathbf{J}} \\
(\mathrm{fF})\end{array}$ & $\begin{array}{c}\mathbf{Z B R} \\
(\mathrm{k} \Omega)\end{array}$ & $\begin{array}{c}\mathbf{R}_{\mathrm{L}} \\
(\mathrm{k} \Omega)\end{array}$ & Remarks \\
\hline 1 & 6.0 & $3 \pm 0.5$ & 6.9 & 132 & CuAu lead, meander \\
2 & 32.2 & $12^{1}$ & 44 & 140 & CuAu lead, meander \\
3 & 11.3 & $3 \pm 1$ & 14.8 & 162 & CuAu lead, meander \\
4 & 27 & 3 & 39.5 & 92 & CuAu lead, meander \\
5 & 23 & $4 \pm 1$ & 34.3 & 150 & CuAu lead, meander \\
6 & 8.8 & $6.5 \pm 1$ & 18.4 & 390 & NiCr lead \\
7 & 29.4 & $.5 \pm 1$ & 82 & 350 & NiCr lead \\
8 & 133 & $3 \pm 1$ & 464 & 350 & NiCr lead \\
9 & 82 & $3.5 \pm 1$ & 239 & 340 & NiCr lead \\
\hline
\end{tabular}

Notes:

1. Only small $\mathrm{I}_{\text {bias }}$ tried, so $\mathrm{C}$ estimate probably large

TABLE 7.1 Summary of the experimental results on the small tunnel junction measurements. $R_{J}$ is the tunneling resistance (measured at high bias), $C_{J}$ the junction capacitance inferred from the high-bias voltage offset, ZBR the zero-bias resistance at $T=20 \mathrm{mK}, R_{L}$ the resistance of one lead to the junction, and the remarks refer to the type of resistive lead used. 
tested by adding and removing the noise filters on the current and voltage leads, at all three temperature stages $(300 \mathrm{~K}, 4.2 \mathrm{~K}$ and $20 \mathrm{mK})$. This had no effect on the low-temperature limit of the ZBR.

Another possibility is that the external circuit was still somehow loading the junctions. This was tested with one junction of each lead type by shoring out all but the first $4.5 \mathrm{~mm}$ of each lead with a layer of In (even more of the leads would have been shorted out, except that the leads cannot be shorted together, and they are only $4 \mu \mathrm{m}$ apart over the remaining $4.5 \mathrm{~mm}$ section). This also had no effect on the ZBR; this implies that the external circuit has been effectively isolated by the resistors, and that only the first few millimeters, at most, of the resistors' length affects the junctions.

As was mentioned earlier (see Chapter 5), there is the possibility of heating in the resistive leads, which could cause the flattening in the data. However, the design of the leads was intended to avoid such problems. The magnitude of the current jitter used for the lock-in measurement and the size of the spurious low-frequency noise do not provide enough power to cause heating at the level needed to explain the data; for a $1 \mathrm{nA} \mathrm{sweep,} \mathrm{the} \mathrm{power}$ dissipated by the jitter current at zero bias is about 10-17 $\mathrm{W}$ for junction 7 .

A fourth possibility is that heating in the junction itself is a significant problem. A crude test of this possibility was performed by removing the magnetic field applied to a small-resistance junction (junction 3 in Table 7.1), and measuring the reduction in the magnitude of the superconducting gap as the bias current was increased. The result of this measurement is shown in Fig. 7.4. Extrapolation of this measurement to the power dissipated in the junction during a normal-state measurements indicates that heating should not be a problem. 
Other possible explanations for the ZBR flattening at low temperatures are that spurious low-frequency noise is causing the flattening, or that the junctions have small enough resistance that quantum fluctuations in the junctions themselves are reducing the ZBR. This latter possibility is discussed in more detail below. I now however tum to a theoretical model that appears to reproduce the main features seen in the data. Subsequent to the formulation of this theory, other authors developed more sophisticated approaches which I examine in a later section. 


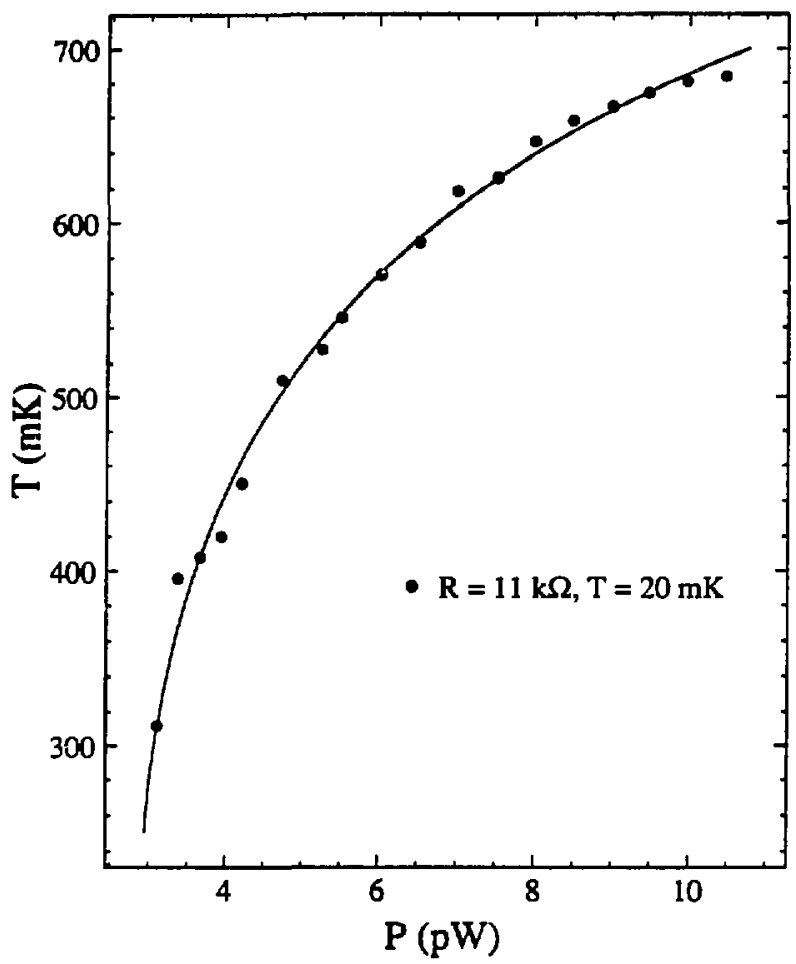

FIGURE 7.4 Junction temperature as a function of dissipated power, as deduced from measurements of the reduction of the superconducting gap as a function of bias current. The refrigerator temperature was $\mathrm{T}=20 \mathrm{mK}$; these data were taken for junction 3 . 


\subsection{Quantum Langevin Theory}

Let us first try to understand what goes on in the circuit shown in Fig. 7.5. This circuit consists of a small tunnel junction with capacitance $C_{J}$ and tunnel resistance $R_{J}$, in series with an ideal resistor $R_{L}$ and a large stray capacitance $C_{\text {stray. }}$. I assume that $C_{\text {stray }} \gg C_{J}$, and $T=0$. Let us place a charge $Q$ on $C_{\mathrm{J}}$; what happens as the charge $Q$ is varied? From the discussion of the isolated small junction, for a charge $|\mathrm{Q}|<\mathrm{e} / 2$ an electron must gain energy in order to tunnel across the junction barrier, and tunneling therefore only occurs for $|\mathrm{Q}|>\mathrm{e} / 2$. If the time an electron takes to tunnel across the junction barrier is $\tau$ (admittedly a rather vaguely defined quantity), and if the circuit time constant $\tau_{R C}=R_{L} C_{J}$ satisfies the equation

$$
\tau_{R C} \gg \tau,
$$

then on the scale of the tunneling time $\tau$, the small junction in Fig. 7.5 acts as if it is isolated from the external circuit. As long as $\tau_{R C} \gg \tau$, the junction should therefore allow electrons to tunnel only for $|\mathrm{Q}|>\mathrm{e} / 2$, and for $|\mathrm{Q}|<$ e/2 no tunneling should eccur.

How difficult is it to achieve the inequality of $E_{q_{1}}$ (7.1)? If I cake the standard time $\tau \approx 10^{-15} \mathrm{~S}(52)$ and a capacitance $C_{J}=10^{-15} \mathrm{~F}$, then even for a resistance $R_{L}=10 \Omega$ I find $\tau_{R C}=10^{-14} \mathrm{~s}$, and the inequality is satisfied! Note however that if we apply the Heisenberg uncertainty relation $\Delta \mathrm{E} \Delta \mathrm{t}>\hbar$, we find that for $\Delta t=10^{-14} \mathrm{~s}$ the charge on the capacitance $C_{J}$ is uncertain by about 20 electrons. We therefore have to consider the effect of quantum mechanics on this system. 


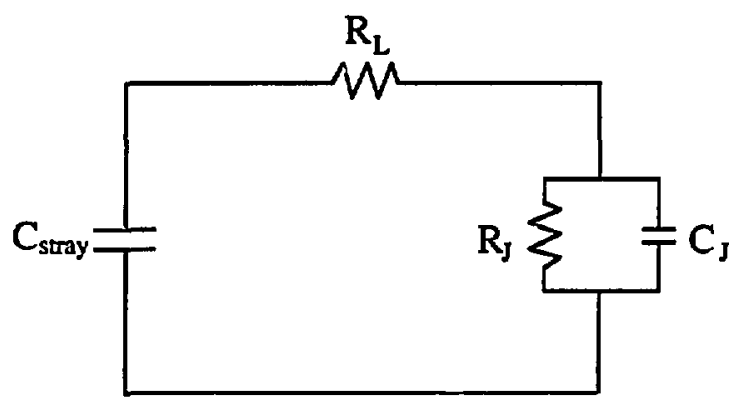

FIGURE 7.5 Simple circuit schematic for a small tunnel junction connected through a large lead resistor $R_{L}$ to low-impedance coaxial lines (not shown) and a large stray capacitance $C_{\text {stray. }}$. The junction tunnel resistance is $R_{J}$ and the capacitance $C_{J}$.

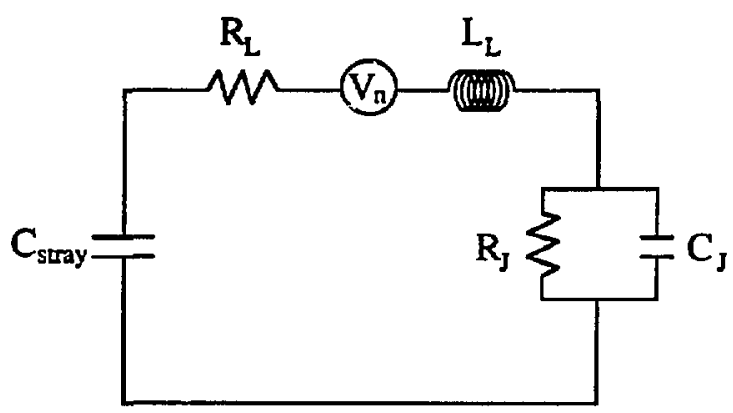

FIGURE 7.6 Circuit model for a small tunnel junction, including the lead resistance $R_{L}$ and lead inductance $L_{L}$, and a large stray capacitance $C_{\text {stray. }}$. The model includes the Johnson-Nyquist voltage noise source $V_{n}$ from the lead resistor. The tunnel junction has tunnel resistance $R_{J}$ and capacitance $C_{J}$. 
The element missing in the discussion above is the voltage noise source $V_{n}$ associated with the lead resistor $R_{L}$, which will have both thermal and quantum contributions to the dehavior of the circuit. The more complete circuit is shown in Fig. 7.6; I have included the lead inductance $\mathrm{L}_{\mathrm{L}}$ for completeness. The voltage source $\mathrm{V}_{\mathrm{n}}$ drives current in the circuit loop, and causes the charge $Q$ to fiuctuate over times much longer than the tunneling time $\tau$. The classical equation of motion for the fluctuations $q(t)$ of the charge $Q$ is given by

$$
L_{L} \frac{d^{2} g}{d t^{2}}+R_{L} \frac{d q}{d t}+\left(\frac{1}{C_{\text {stray }}}+\frac{1}{C_{J}}\right) q=V_{n}(t)
$$

This equation may be Fourier transformed and solved for the mean square fluctuation $\left\langle q^{2}(\omega)\right\rangle$, in terms of the spectral distribution of the voltage noise, $S_{V}(\omega)=\left\langle V_{n}{ }^{2}(\omega)\right\rangle$ :

$$
<q^{2}(\omega)>=\frac{C_{\mathrm{J}}^{2}}{\left(1-\omega^{2} / \omega_{L} c^{2}\right)^{2}+\left(\omega / \omega_{R C}\right)^{2}} S_{V}(\omega)
$$

Here I have defined tha ifrequencies $\omega_{R C}=1 / R_{L} C_{J}$ and $\omega_{L C}=1 / \sqrt{L_{L} C_{J}}$, and I have dropped the stray capacitance with the assumption $\mathrm{C}_{\text {stray }} \gg \mathrm{CJ}_{\mathrm{J}}$. II order to explain the behavicr of the zero-bias resistance at low temperatures, I use the spectral distribution of voltage noise given by the full JohnsonNyquist formula $(53,54)$; this is given by

$$
S_{V}(\omega)=\frac{\hbar \omega R_{L}}{\pi} \operatorname{coth}\left(\hbar \omega / 2 k_{B} T\right)
$$


This expression includes the standard low-frequency $\left(\hbar \omega<<k_{\mathrm{B}} \mathrm{T}\right)$ result $S_{V}(\omega)=2 k_{B} T R_{V} / \pi$, and at high frequencies includes the quantum fluctuations, $S_{V}(\omega)=\hbar \omega R_{I} / \pi$. Even at $T=0$ there are significant voltage fluctuations caused by the resistor. Using the Wiener-Khinchine theorem, I can write the instantane'sus charge fluctuation $\left\langle q^{2}(t)>\right.$ in terms of the frequency-domain fluctuations,

$$
\left\langle q^{2}(t)\right\rangle=\int_{0}^{\infty}\left\langle q^{2}(\omega)\right\rangle d \omega .
$$

The integral in Eq. (7.5) may be performed analytically in two limits; at very high temperatures the result is the standard expression from equipartition of energy, $\left\langle q^{2}>/ 2 C_{J}=k_{B} T / 2\right.$. With the parameter $\alpha=\omega_{\mathrm{LC}} / \omega_{\mathrm{RC}}$, I find in the limit of small $\mathrm{T}$ and for $\alpha<2$,

$$
\frac{\left\langle\mathrm{g}^{2}\right\rangle}{2 C_{\mathrm{J}}}=\frac{\hbar \operatorname{arc}}{2 \pi} \frac{1}{\sqrt{4-\alpha^{2}}}\left(\frac{\pi}{2}-\tan ^{-1}\left(\frac{\alpha^{2}-2}{\alpha \sqrt{4-\alpha^{2}}}\right)\right) .
$$

For very small $\alpha$ I get the simple result $\left\langle q_{1}^{3}\right\rangle / 2 \mathrm{C}=\hbar \omega_{L} d 4$, as expecited for a simple harmonic oscillator with no dissipation. For $\alpha>2$ and small $\mathrm{T}$ I find

$$
\frac{\left\langle q^{2}\right\rangle}{2 C_{J}}=\frac{k \omega_{L C}}{4 \pi}-\frac{1}{\sqrt{4-\alpha^{2}}} \ln \left(\frac{\alpha^{2}-2+\alpha \sqrt{\alpha^{2}-4}}{\alpha^{2}-2-\alpha \sqrt{\alpha^{2}-4}}\right) .
$$

In the limit of very largs $R_{L}$ I find that $\left\langle q^{2}\right\rangle / 2 C=\left(\hbar \omega_{R} c / \pi\right)$ in $\alpha$, which falls as $1 / R_{L}$ and has only a logarithmic dependence on $L_{L}$. In Fig. 7.7 I display the dependence of $\left\langle q^{2}>/ \mathrm{e}^{2}\right.$ on $R_{\mathrm{L}}$ for $\mathrm{L}_{\mathrm{L}}=4.5 \mathrm{nH}$ and $\mathrm{C}_{\mathrm{J}}=4.5 \mathrm{fF}$ at $\mathrm{T}=0$, the 
inductance and capacitance being those that apply to the first $4.5 \mathrm{~mm}$ of the resistive leads in the experiment. In Fig. $7.8 \mathrm{I}$ display the temperature dependence of $\left\langle q^{2}\right\rangle / e^{2}$ for $L_{L}=4.5 \mathrm{nH}, C_{J}=4.5 \mathrm{fF}$ and $R_{L}=9.0 \mathrm{k} \Omega$ and 130 $\mathrm{k} \Omega$, the latter being the resistance for the first $4.5 \mathrm{~mm}$ of the $\mathrm{CuAu}$ and $\mathrm{NiCr}$ leads, respectively.

The value of $\left\langle q^{2}\right\rangle$ is the spread of the distribution $P(q)$, which describes the probability of having a charge fluctuation of size $q$ on the small junction capacitance. In the discussion of section 5.1, I assumed the probability distribution to be $P(q)=\delta(q)$. The spread in the possible values of $q$ comes from both thermal and quantum fluctuations. In the limit of zero temperature, the probability distribution wonld be the square of the wavefunction, or probability amplitude, of the variable $q$.

In order to accommodate the spread in the values of $q$ in the calculation of the current-voltage characteristic, I assume that the effective tunneling rate $\langle\Gamma(Q)>$ of electrons will be the tunneling rate $\Gamma(Q)$ in the absence of fluctuations, convolved with the probability $P(q)$ of a given size fluctuation g. As the fluctuations are concentrated at frequencies much below $1 / \pi$, this seems a reasonable approximation. The resulting expression is

$$
\langle\Gamma(Q)\rangle=\int_{-\infty}^{\infty} \Gamma(Q+q) P(q) d q
$$

An analytic expression for $\Gamma(Q)$ is given by (55),

$$
\Gamma \pm(Q)=-\frac{e / 2 \pm Q}{e R_{J} C_{J}}(\exp (\Delta E \pm / k T)-1)^{-1}
$$




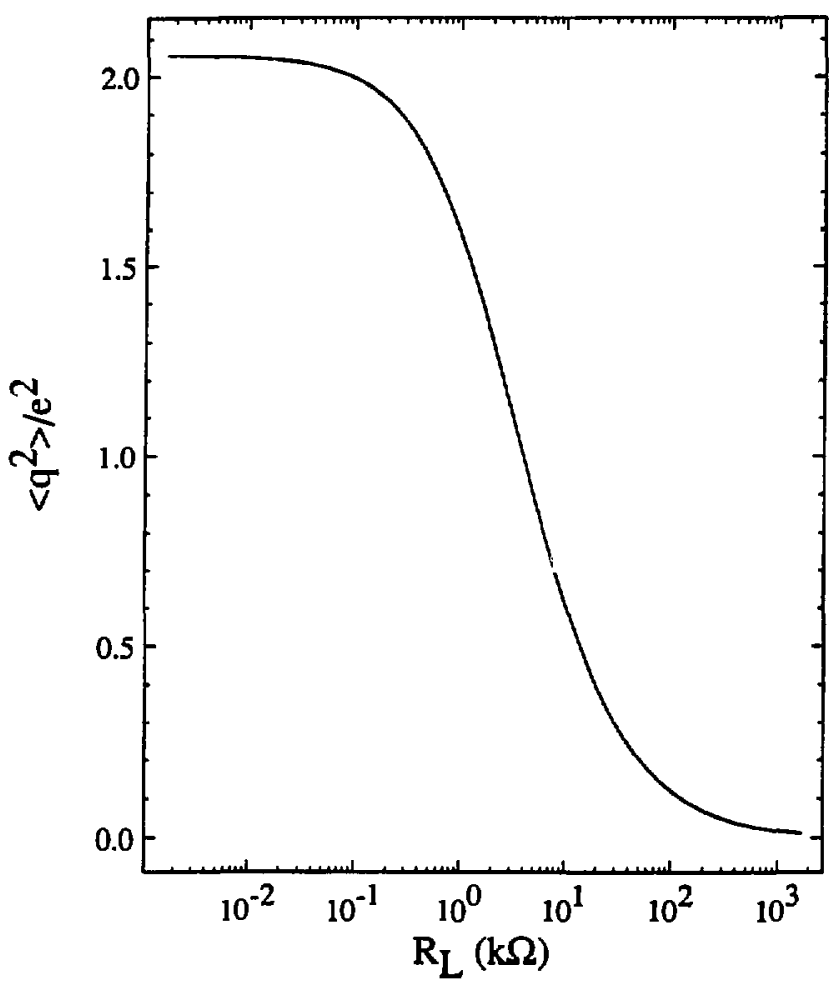

FIGURE 7.7 Plot of the dependence of $\left\langle q^{2}>/ e^{2}\right.$ on $R_{L}$ for junction capacitance $C_{J}=4.5 \mathrm{fF}$, lead inductance $\mathrm{L}_{\mathrm{L}}=4.5 \mathrm{nH}$ and at $\mathrm{T}=0$, calculated using the quantum Langevin theory. 


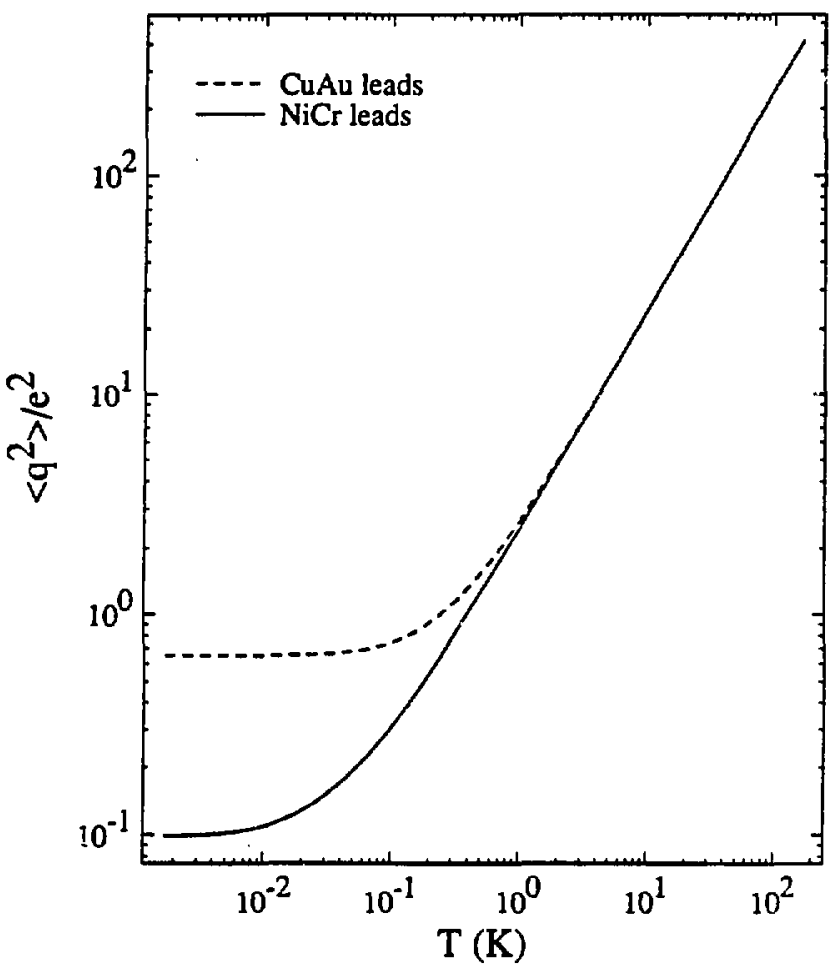

FIGURE 7.8 Plot of the temperature dependence of $\left\langle q^{2}>/ e^{2}\right.$, as calculated from the quantum Langevin theory, for both the $\mathrm{CuAu}$ and $\mathrm{NiCr}$ leads; see text for circuit parameters. 
Here $\Gamma^{+}$is the rate for $Q$ to go to $Q+e$, and similarly $\Gamma^{-}$is the rate for $Q$ to become $\mathrm{Q}-\mathrm{e}$. The calculations for tunneling rates are done independently for the two processes, although the same result is achieved using the definition $\Gamma$ $=\Gamma^{+}-\Gamma^{-}$. The probability distribution $P(q)$ is assumed to be Gaussian, chosen to have unit normalization and the correct mean square width:

$$
P(q)=\frac{1}{\sqrt{\left.2 \pi<q^{2}\right\rangle}} \exp \left(-\frac{q^{2}}{2\left\langle q^{2}\right\rangle}\right) \text {. }
$$

The convolution of Eq. (7.8) can be carried out analytically at $T=0$. The result is given by

$$
\begin{aligned}
\left\langle\Gamma^{ \pm}\right\rangle= & -\frac{e / 2 \pm Q}{2 e R_{J} C_{J}} \operatorname{erfc}\left(\frac{e / 2 \pm Q}{\sqrt{\left.2<q^{2}\right\rangle}}\right) \\
i \quad & +\frac{1}{e R_{J} C_{J}} \sqrt{\frac{\left\langle q^{2}\right\rangle}{2 \pi}} \exp \left(-\frac{(e / 2 \pm Q)^{2}}{2\left\langle q^{2}\right\rangle}\right) .
\end{aligned}
$$

In Fig. 7.9 I plot $<\Gamma(Q)>$ and $\Gamma(Q)$ for $\left\langle q^{2}>=0.65 \mathrm{e}^{2}\right.$ and $0.098 \mathrm{e}^{2}$, the values for the $\mathrm{CuAu}$ and $\mathrm{NiCr}$ leads, at $\mathrm{T}=0$, to show the smearing of the Coulomb gap due to the uncertainty in the value of $Q$. Note that at large values of $Q$, the two rates are identical; the smearing only occurs for values of $Q$ within $\sqrt{\left\langle q^{2}\right\rangle}$ of $\pm e / 2$.

From the discussion above, the main experimental features of the data can now be explained by this simple model. The smearing of the Coulomb gap at low bias voltages is caused by fluctuations in the leads, and the smearing becomes less apparent as the fluctuations are reduced in magnitude by increasing the value of the lead resistance, or by reducing the temperature. We furthermore see that the zero temperature limit of the 
current-voltage characteristic can be explained by the inclusion of the zeropoint quantum fluctuations in the leads. In order to make a more definite comparison, I have to choose parameters for the simple circuit model I have used in this calculation. The quantum Langevin approach will unfortunately not work for the more complete resistive transmission line model of the leads. In order to make a somewhat reasonable approximation to the actual circuit, I choose to include only the first $4.5 \mathrm{~mm}$ of each lead, and use the calculated resistive transmission line elements in a lumped fashion as the elements of the model circuit used here. In other words, I choose the total inductance to be $4.5 \mathrm{nH}$, and the resistance to be $9 \mathrm{k} \Omega$ for the $\mathrm{CuAu}$ leads and $130 \mathrm{k} \Omega$ for the $\mathrm{NiCr}$ leads. The junction capacitance was chosen from the high-bias limit of the Coulomb offset for the two junctions; according to the theory sketched out above, this should be correct even in the presence of fluctuations.

One part of the experimental situation which I have thus far ignored is that the measurements reported here were done with a current bias, not a voltage bias. All the tunneling rates calculated here are for a fixed voltage bias. In order to calculate the current-biased average voltage, I carried out an approximate simulation of the junction behavior. The method is outlined in Appendix D. The idea is to approximate the stochastic tunneling of electrons by a periodic tunneling sequence, and use the circuit of Fig. 7.5 to calculate the time dependent voltage $\mathrm{V}(\mathrm{t})$. The average voltage is then calculated for each value of current bias $\mathrm{I}_{\text {bias, }}$ and the cur"ent Ibias swept over the range of interest to give a full current-voltage characteristic. Note that at different values of current bias, the time-dependent voltage has a characteristically different shape, as shown in Appendix D, Fig. D.3. At low values of bias current, the sawtooth pattern can in principle be locked into an 


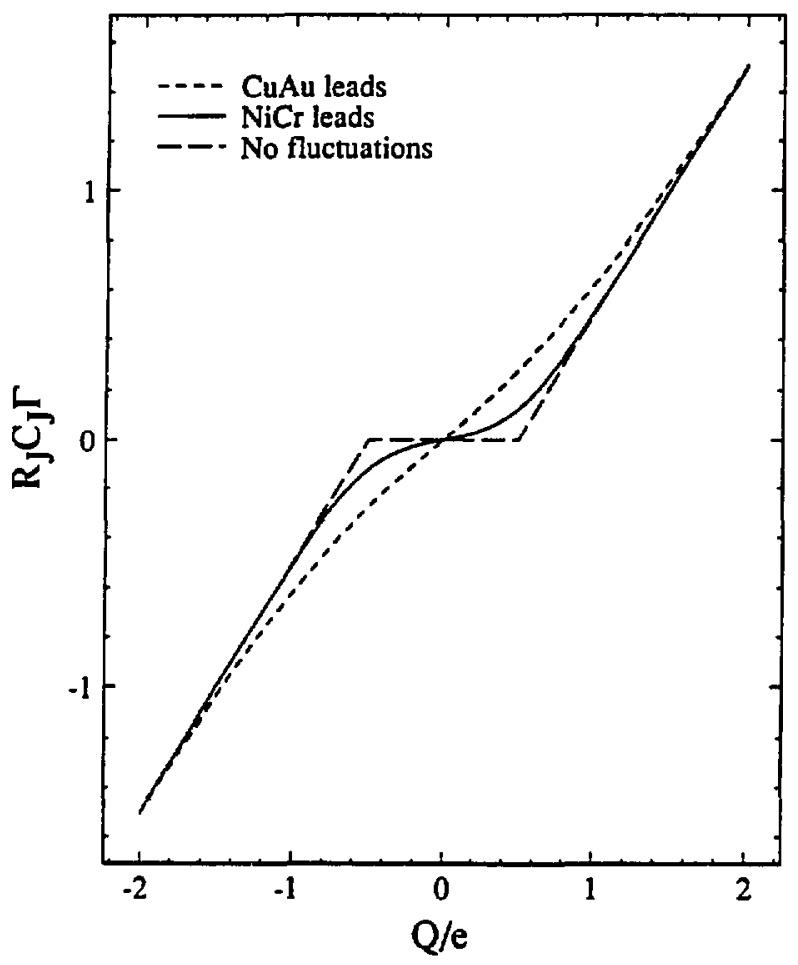

FIGURE 7.9 Comparison of the $T=0$ tunneling rate $\Gamma$ as predicted by the quaitum Langevin theory, as a function of junction charge $\mathrm{Q} / \mathrm{e}$. The dotted curve is for the CuAu leads and the solid curve for the $\mathrm{NiCr}$ leads, with the circuit parameters given in the text. The dashed curve is the tunneling rate in the absence of fluctuations. The tunneling rate $\Gamma$ is normalized to the highbias tunneling rate $R_{J} C_{j}$ of the junction. 
$\mathrm{RF}$ current signal and give locked current steps analogous to the Josephson ac effect (19). The time-averaged voltage $\langle V\rangle$ is much easier to measure than the time-dependent voltage.

In Fig. 7.10 I plot the comparison of the theory at $T=0$ and experimental data. The data for the $\mathrm{CuAu}$ leads are in reasonable agreement at low bias and less good agreement at high bias, while the data for the $\mathrm{NiCr}$ leads show reasonable agreement over the entire range of displayed bias currents. In Fig. 7.11 I show the ZBR as a function of temperature, for both the theory and for the experiment. The general features of the data are clearly present in the theory, with the ZBR rising as the temperature is lowered, until below some temperature the data and theory flatten off. The experimental results are not reproduced exactly by the theory, but the general trends are clearly there.

This theory is clearly a heuristic approach to the full problem of how the environment affects small tunnel junctions. It seems to reproduce the broad experimental features, and fitting the circuit parameters used in the theory (for instance the length of lead to include) could lead to a much better agreement. Rather than pursue this further, I now tum to a description of the more rigorous theories involving the phase correlation function, which are able to handle a more general impedance than that of the approximate RLC circuit, and also treat the motion of the phase variable in a rigorous fashion. 

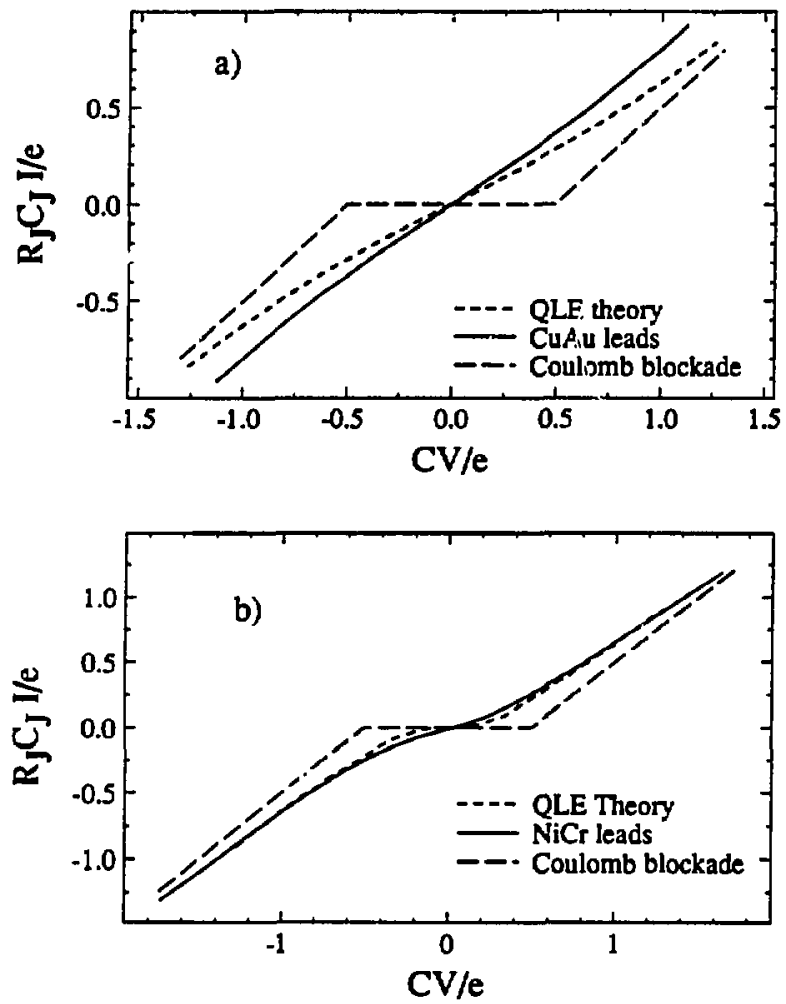

FIGURE 7.10 Comparison of the quantum Langevin theory with the experimental current-voltage characteristics; (a) is junction 5 with $\mathrm{CuAu}$ leads and (b) is junction 7 with $\mathrm{NiCr}$ leads. The solid dots are the quantum Langevin equation (QLE) predictions at $T=0$, and the dashed line the predicted voltage-biased Coulomb blockade. 


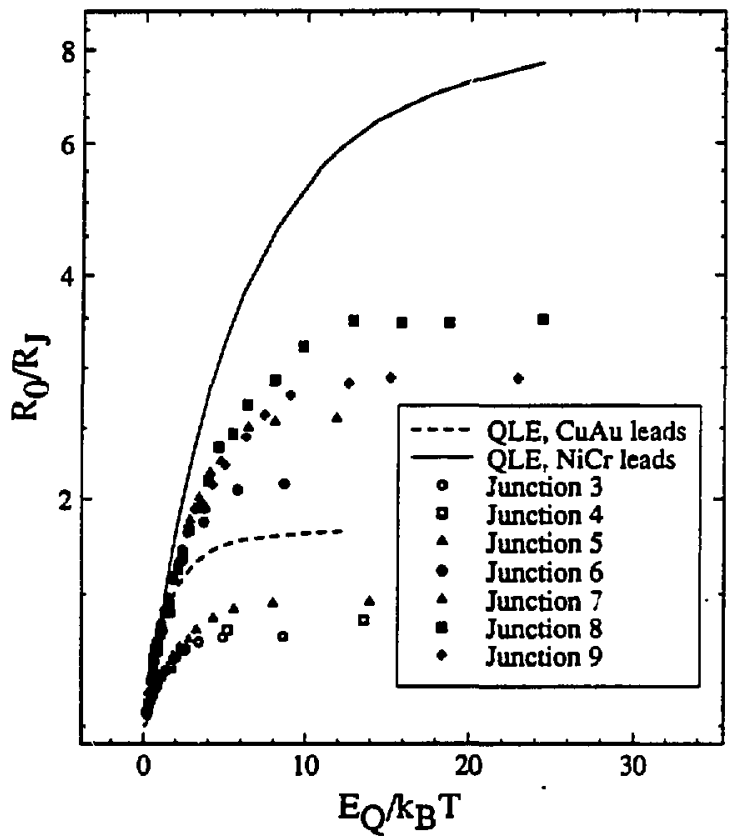

FIGURE 7.11 Zero-bias resistance preticted by the quantum Langevin theory compared with that measured experimentally. Symbols are as in Fig. 7.3 


\subsection{Phase Correlation Theory}

Several authors have published different versions of an alternative theory to the quantum Langevin theory presented here $(28,56,57)$. I will describe the version of the therry described by Devoret et al. (28).

The fundamental idea behind the small junction theory presented in section 5.1 is that the Hamiltonian for the system can be written as

$$
H=\frac{Q^{2}}{2 C}+H_{T}+H_{\text {res }} \text {, }
$$

where $\mathrm{C}$ is the junction capacitance, $\mathrm{H}_{\mathrm{T}}$ the tunnel Hamiltonian that describes single electron transfer, and $\boldsymbol{H}_{\text {res }}$ the model Hamiltonian for the external circuit. The term giving the single electron transfer is usually written as

$$
H_{T}=\sum_{\text {नkq }} M_{k q} c_{k \sigma}{ }^{*} c_{q \sigma}+H . C .
$$

where $\mathbf{M}_{\mathrm{kq}}$ is the transfer matrix element and $c_{\mathrm{k} \sigma}$ the destruction operati:. for electrons of spin $\sigma$ on one electrode; the summations over $k$ and $q$ are over all wavevectors in both electrodes. In that section, the loss of energy of an electron tunneling across the junction was treated in a rather $a d$ hoc fashion. Devoret et al. (28) recognized that this energy loss could be included by rewriting the expression for $\mathrm{H}_{T}$ as

$$
H_{T}=\sum_{\sigma k q} M_{k q} \varepsilon_{k \sigma}{ }^{*} c_{q \sigma} e^{i \delta}+\text { H.C. . }
$$

where $\delta$ is the phase variable conjugate to the charge operator $Q$ : 


$$
\delta=\frac{e}{h C} \int Q d t
$$

The phase exponent in Eq. (7.14) forces the inclusion of the charging energy in the tunneling process because of the commutator relation

$$
e^{i \delta} e^{-i \delta}=Q-e
$$

With this revised expression for the tunneling Hamiltonian, a calculation can be performed to obtain the tunneling rate as a function of junction voltage. The calculation involves the phase correlation function $\mathrm{J}(\mathrm{t})$, given by

$$
J(t)=<[\delta(t)-\delta(0)] \delta(0)>,
$$

which can be calculated for a general circuit impedance $Z(\omega)$. At a temperature $T$, the phase correlation function is given by

$$
J(t)=\int_{0}^{\infty} \frac{d \omega}{\omega} \frac{\operatorname{Re} Z_{1}(\omega)}{R_{Q}}\left\{\operatorname{coth}\left(\frac{\beta \hbar \omega}{2}\right)[\cos (\omega t)-1]-i \sin (\omega t)\right\},
$$

where $I$ use the inverse temperature $\beta=1 / \mathrm{k}_{\mathrm{B}} T$, the total impedance function $Z_{i}(\omega)$ is given by $Z_{i}^{-1}(\omega)=i \omega C+Z^{-1}(\omega)$; and the normal-metal resistance quantum is $R_{Q}=h / 2 e^{2}=12.91 \mathrm{k} \Omega$. Knowledge of the phase correlation function allows calculation of the probability $P(E)$ of an energy fluctuation of size $\mathrm{E}$ through the expression 


$$
\mathrm{P}(\mathrm{E})=\frac{1}{2 \pi \mathrm{h}} \int_{-\infty}^{\infty} \mathrm{dt} \exp (\mathrm{J}(\mathrm{t})+i \mathrm{Et} / \mathrm{h})
$$

Just as in the discussion of the quantum Langevin equation, the probability $P(E)$ is assumed to be a $\delta$-function for a fluctuation-free environment. Given the actual probability distribution for a real environment, the tunneling rate $\Gamma$ at a voltage $\mathrm{V}=\mathrm{Q} / \mathrm{C}$ can be calculated from the expression

$$
\Gamma(V)=\frac{1}{e^{2} R_{J}} \int_{-\infty}^{\infty} d E E \frac{1-\exp (-\beta e V)}{1-\exp (-\beta E)} P(e V-E)
$$

for a junction with tunnel resistance $R_{\mathrm{J}}$. This derivation assumes that quantum fluctuations in the junction itself can be neglected, i.e. that $R_{\mathbf{J}}$ is much larger than the resistance quantum $\mathrm{R}_{\mathrm{Q}}$. This question is discussed below.

The calculation of Eq. (7.20) gives the voltage-biased tunneling rate $\Gamma(V)$; just as for the case of the quantum Langevin equation discussed in section 7.3, we have to account for the actual biasing circuit used in the experiment. We have chosen to use the same approximate method described in Appendix $D$, using a fixed time $\Delta t=e / I_{\text {bias }}$ between tunneling events. However, we replace the simple RLC circuit of section 7.3 with the impedance $Z_{t}(\omega)$ of the actual biasing circuit (see Appendix D).

We have applied this theory to the measurements on the small junctions reported earlier. The calculations involve the complete model for the resistive transmission line, using the parameters discussed earlier (see Chapter 5). The comparison of the measured I-V characteristics at $T=20$ 
$\mathrm{mK}$ with the theoretical predicsions at $\mathrm{T}=20 \mathrm{mK}$ are shown in Fig. 7.12 (a) and (b). In order to get better agreement for this figure, as well as for the differential resistance comparison, the temperature for the theory was increased to $38 \mathrm{mK}$ for the CuAu leads and to $28 \mathrm{mK}$ for the $\mathrm{NiCr}$ leads; this comparison is shown in Fig. 7.12 (c) and (d), and the differential resistance compared in Fig. 7.13. We see that for the junction with the CuAu leads the experiment and theory match well at low bias currents, seen in the I-V characteristic, but that at higher bias currents the predicted differential resistance is much sharper than that observed. The junction with the $\mathrm{NiCr}$ leads however matches much better, with only slight deviations seen in the I$\mathrm{V}$ characteristic and in the differential resistance. The temperature dependence of the zero-bias resistance (ZBR) is compared in Fig. 7.14. We see that at high temperatures the ZBR from experiment and theory match quite well, but as the temperature is lowered the data flatten out while the theory continues to rise. As discussed earlier, several attempts were made to. explain the experimental zero-bias behavior in terms of spurious noise and heating, none of which were shown to have any effect on the measurements. As it stands, therefore, there is no good explanation for the discrepancy between the ZBR predicted by the theory and that observed in the experiment.

There is a possible explanation, however, involving the quantum flucuuations in the junction itself. A paper by Brown and simanek (58) discussed the effect of the junction tunnel resistance $R_{J}$ on the $Z B R$ at low temperatures. An experiment by Mooij's group (26) appears to confirm their calculation. The basic result of the theoretical calculation, whicin uses a formalism similar to $\mathrm{s}$ that of Devoret et al. (28), is that the zero-bias conductance $Y(0)$ is given by 


$$
Y(0)=\frac{1}{\pi R_{J}} \int_{0}^{\pi} \Gamma_{0}(x) d x,
$$

in terms of the phase correlation function $\Gamma_{0}(x)$, which is given by the selfcorsistent solution of the expression

$$
\ln \Gamma_{0}(x)=-a\left(\sum_{n=1}^{\infty} \frac{1-\cos (n x)}{n^{2}+a g(2 / \pi) \int_{0}^{\pi} \Gamma_{0}(x) \frac{1-\cos (n x)}{1-\cos (x)} d x}\right)
$$

with the consiants $a=e^{2} / 2 \pi^{2} C_{j} k_{B} T$ and $g=R_{Q} / 2 R_{N}$. In the limit $g=0$, this may be solved analytically to give

$$
\Gamma_{0}(x, g=0)=\exp \left(-a\left(-\frac{x^{2}}{4}+\frac{\pi x}{2}\right)\right)
$$

This analytic solution is used as a starting point to calculate numerically a value of $\Gamma_{0}(x)$ for non-zero values of $\mathrm{g}$, by iterating Eq. (7.22). Equation (7.21) is then used to calculate the zero-bias resistance. Figure 7.15 shows the zero-bias resistance $R_{0}$ as a function of junction turuneling resistance $R_{J}$, for a fixed reducid temperature $\mathrm{E}_{\mathrm{Q}} / \mathrm{k}_{\mathrm{B}} \mathrm{T}=10$. Also plotted is the interpolated value of $\mathrm{R}_{0} / \mathrm{R}_{\mathrm{J}}$ for the junctions connected to the $\mathrm{NiCr}$ leads. The theory shows the same trend as the experiment, although agreement is by no means exact. It seems possible that a theory combining the effects of a finite impedance environment with a finite junction tunneling resistance might agree more closely with the experiment. 

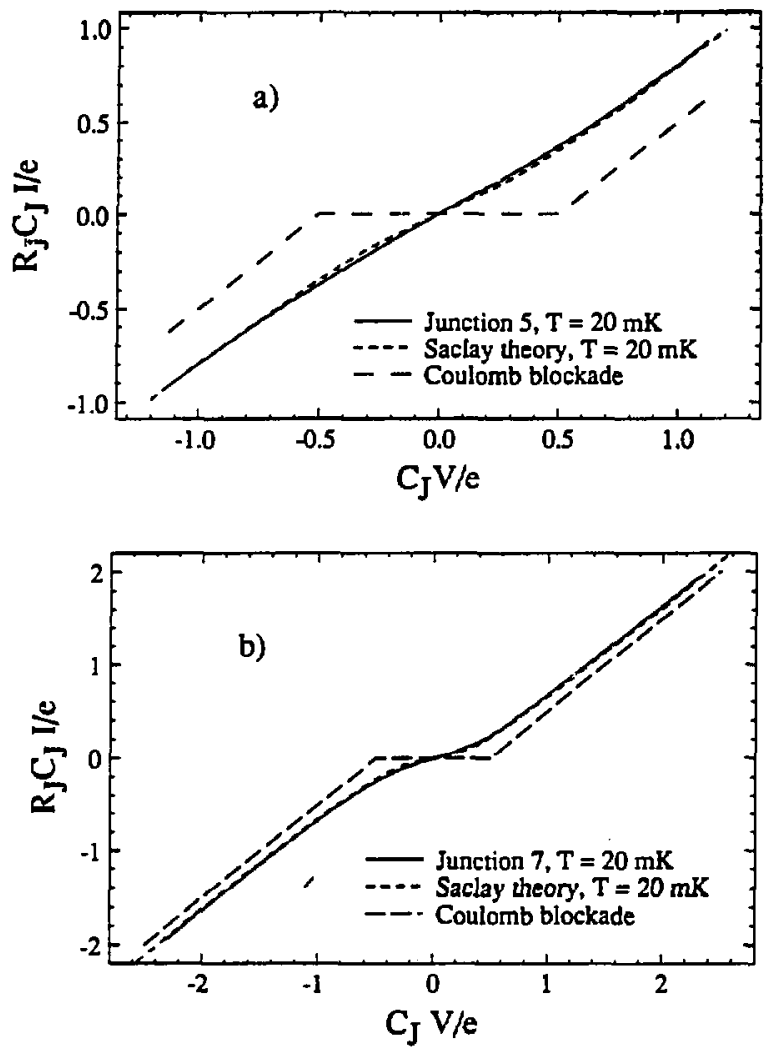

FIGURE 7.12 (a) and (b). Comparison of the theory of Devoret et al. (28) with experimental current-voltage characteristics; (a) is junction 5 and (b) is junction 7 . The theory, shown by the dashed lines, and the data, shown by solid lines, are at $\mathrm{T}=20 \mathrm{mK}$. The calculations were performed with the resistive transmission line model, using calculated circuit parameters with no fitting. The voltage-biased Coulomb blockade is also shown. 

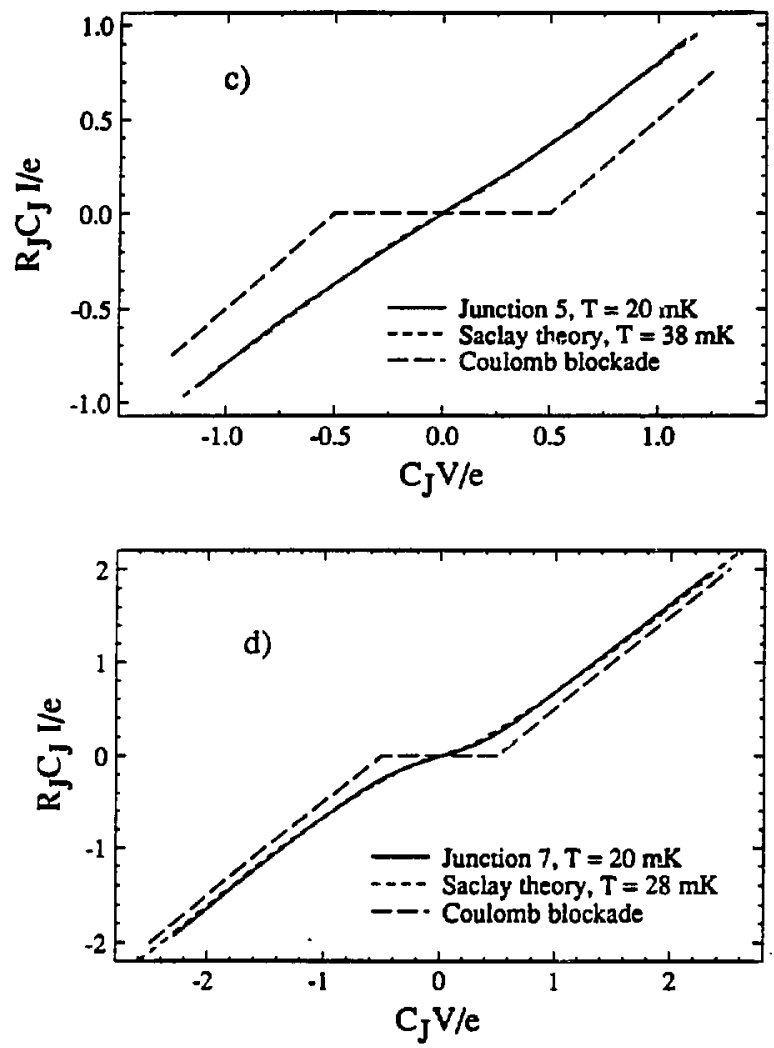

FIGURE 7.12 (c) and (d). Comparison of the theory of Devoret et al. (28) with experimental current-voltage characteristics; (c) is junction 5 and (d) is junction 7. The data, shown by solid lines, are at $\mathrm{T}=20 \mathrm{mK}$, and the theory is at the $38 \mathrm{mK}$ for (c) and $28 \mathrm{mK}$ for (d). The calculations were performed with the resistive transmission line model, using calculated circuit parameters with the temperature adjusted for better fit. The voltage-biased Coulomb blockade is also shown. 

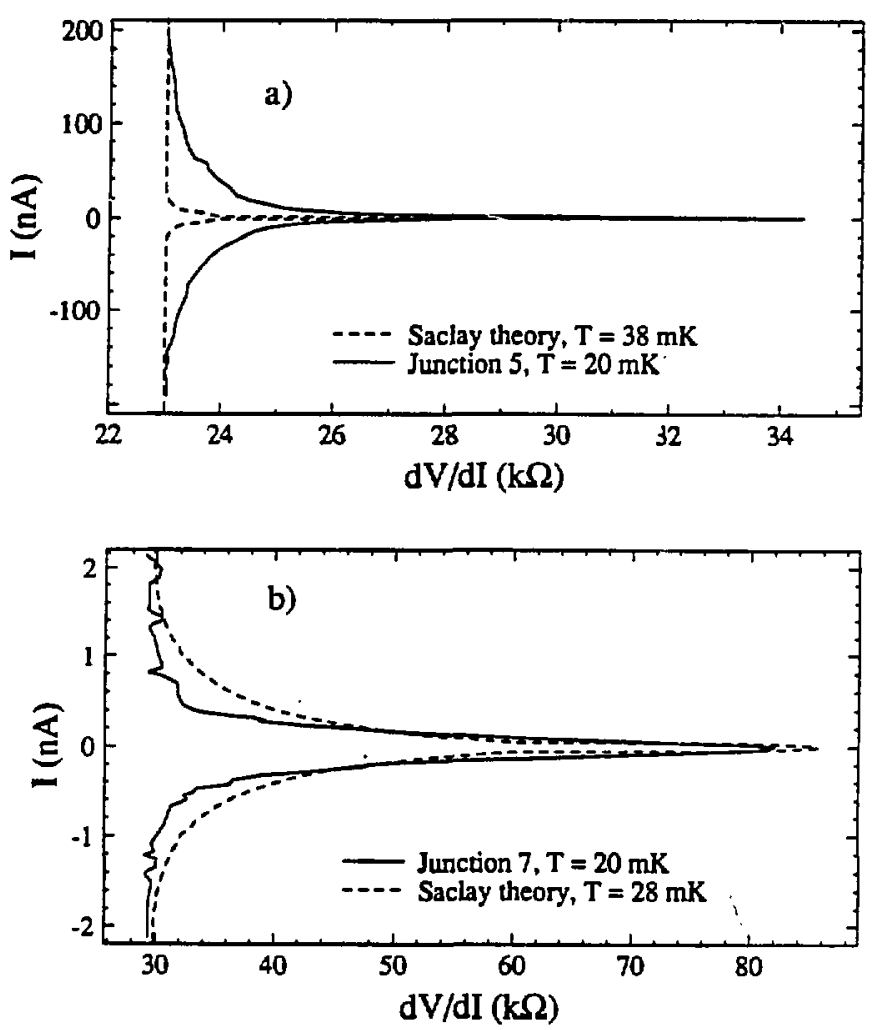

FIGURE 7.13 Comparison of the theory of Devoret $e$ al. (28) with experimental differential resistance measurements; (a) is for junction 5 and (b) is for junction 7. The data (solid lines) are at $T=20 \mathrm{mK}$, while the theory (dashed lines) are at $\mathrm{T}=38 \mathrm{mK}$ for (a) and $\mathrm{T}=28 \mathrm{mK}$ for (b). 


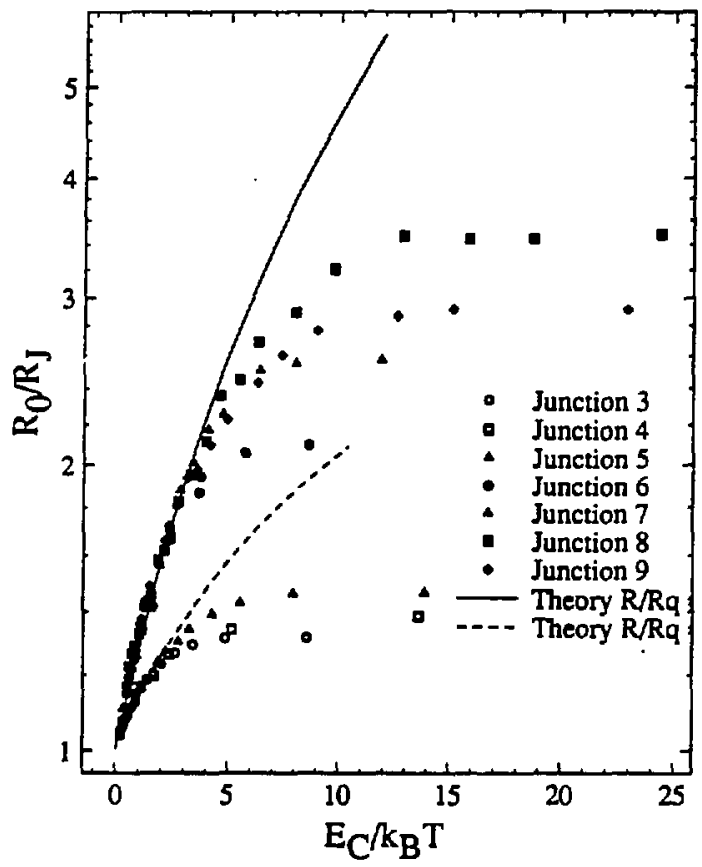

FIGURE 7.14 Zero-bias resistance predicted by the theory of Devoret et al. (28), using the circuit parameters for the $\mathrm{CuAu}$ leads (dashed line) and the $\mathrm{NiCr}$ leads (solid line). 


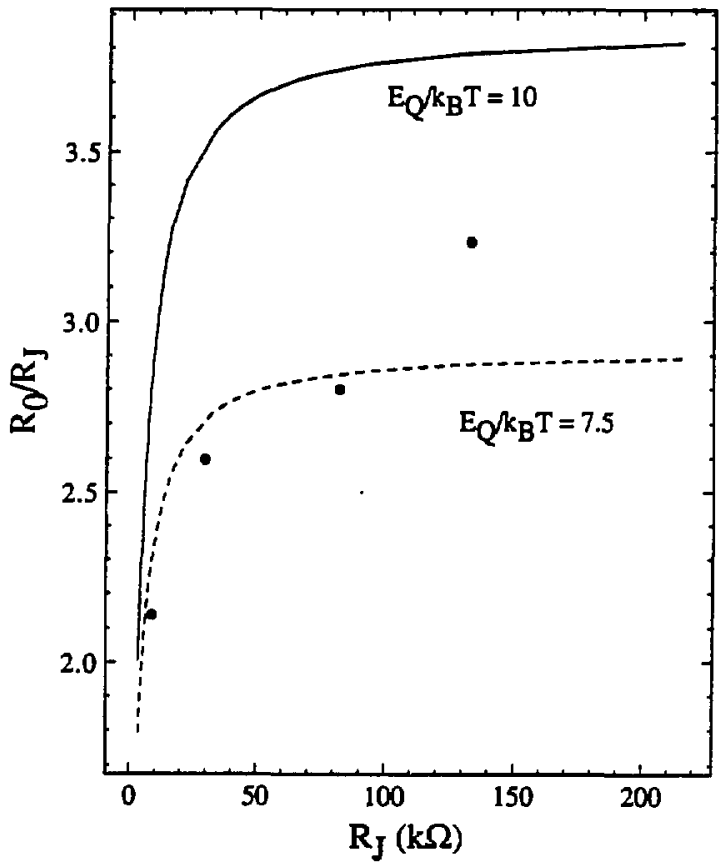

FIGURE 7.15 Zero-bias resistance $R_{0} / R_{J}$ as a function of junction tunnel resistance RJ calculated using the theory of Brown and Simanek. The calculation was performed using the reduced temperatures $E_{Q} / k_{B} T=7.5$ and EQ/kBT $=7.5$. 


\title{
CHAPTER 8
}

\author{
Conclusions
}

In this final chapter I summarize briefly the results for the MQT and small junction experiments, and try to show how the two experiments together illustrate a basic result of the effect of quantum mechanics on electrical circuits. 
In the preceding chapters I have discussed the application of quantum mechanics to a rather unusual system: the description of a macroscopic variable, the phase variable of a superconducting junction and the charge on a small capacitance tunnel junction.

In the case of the phase variable, I discussed measurements of the escape of the phase variable from a local metastable potential minimum, either by thermal activation over the top of the potential barrier or by quantum tunneling through the barrier. The Josephson junction used in the measurement was shunted by an external metallic resistor with a resistance of $9.3 \Omega$, giving a quality factor for oscillations in the bottom of the well of $Q=1.7$. This low value of $Q$ was shown to reduce the zero-temperature quantum tunneling rate by a factor of 300 from the tunneling rate for a system with $Q=\infty$, in very good agreement with the theoretical predictions. Furthermore, the temperature dependence of the escape rate, in the presence of this moderate dissipation, was shown to follow the predictions quite well from $\mathrm{T}=0$ to $\mathrm{T} \gg \mathrm{T}_{\mathrm{cr}}$, where the process of thermal activation is well described by completely classical equations. An important part of the measurements described here is that all the parameters of the junction (the critical current, the shunt resistance, and the junction capacitance), were measured in the classical limit by classical processes. No assumptions involving the quantum limit were necessary in analyzing the data. The results presented here therefore provide strong support both for the application of quantum mechanics to this rather unusual system, and for the model of Caldeira and Leggett for a dissipative element, a model which applies in both the classical and quantum limits.

The theoretical and experimental investigations of the quantum behavior of the josephson junction led historically to the investigation of the 
"extreme quantum limit" reached in a Josephson junction when the junction capacitance $C$ is reduced to the femtofarad $\left(10^{-15} \mathrm{~F}\right)$ range. The phase of the junction, defined in either the normal or superconducting state, becomes less sharply known. The conjugate variable to the phase, the charge on the junction, becomes suuch more shaply defuned. The description of the junction changes in a fashion analogous to that of an electron whose environment changes from a tight-binding potential to that of free space; momentum becomes a "better" dynamic variable than position. A simple theory, applicable to a normal junction, predicts the appearance of a infiniteresistance portion of the current-voltage characteristic for voltages between $-\mathrm{e} / 2 \mathrm{C}$ and $\mathrm{e} / 2 \mathrm{C}$. The measurements made here, where different single small junctions were connected to leads including resistors designed to work at high frequencies, illustrated the need for the theory to include the external impedance presented by the junction environment. A simple theory using the quantum Langevin formalism was developed, which was in rough agreement with the measurements. The main points of the theory were to include the resistors in the description of the junction behavior, and to include both the thermal and quantum fluctuating currents driven through the small tunnel junction by the resistor voltage noise.

Other theories were published after the measurements described here were reported. These more complete theories are however still limited to junctions with infinite tunnel resistanca. Comparison of the predictions of these theories with the measurements show good agreement over the highercurrent portions of the I-V characteristic, but poor agreement at low bias currents. The zero-bias resistance (ZBR) follows the predictions reasonably well at high temperatures, but at the lowest temperatures the data shown a flattening of the ZBR not seen in the theory. A possible resolution of this 
difficulty is provided by the theory of Brown and Simanek, which describes the effects of finite junction tunnel resistance on the junction ZBR. There remains a need for a theory which includes both the effects of finite environment impedance and finite junction tunnel resistance. It is also possible that the discrepancies are due to ununticipated experimental difficulties.

The experiments on the shunted Josephson junction and the smallcapacitance tunnel junctions together provide insight into the behavior of quantum-mechanical systems, which involve the effects of the external environment in an essential way. The experiments illustrate the possibility of applying quantum mechanics to any system for which a classical description exists. New phenomena, due to the quantized nature of the variables describing the system, may then be observable. 
APPENDICES 


\section{Appendix A - Capacitance Calculations}

The calculations of the capacitances involved in the small tunnel junction experiments, both the lumped capacitance associated with the junction overlap area itself, and the distributed capacitances of the leads to ground and to each other, are complicated by the question of which capacitances are important. I will pose a gedanken experiment to illustrate the difficulties involved.

Let us take the simple case of two concentric spheres of radii $r$ and $R$, with $r<R$. The capacitance for a single sphere of radius $r$ is $C=4 \pi \varepsilon_{0} r$ (in MKS units); this is the capacitance to the ground at infinity. If $I$ add another sphere to the problem, I now have to deal with a capacitance matrix which relates the charge $Q_{i}$ to the voltage $V_{i}$ on the $i^{\text {ith }}$ sphere:

$$
\left(\begin{array}{l}
Q_{r} \\
Q_{R}
\end{array}\right)=\left(\begin{array}{ll}
C_{\pi r} & C_{r R} \\
C_{R r} & C_{R R}
\end{array}\right)\left(\begin{array}{l}
V_{r} \\
V_{R}
\end{array}\right)
$$

Note that $C_{r R}$ is always equal to $C_{R r}$, by symmetry. All the elements in the capacitance matrix are easily calculated; the result is given by

$$
\left(\begin{array}{cc}
C_{r} & C_{r R} \\
C_{R s} & C_{R R}
\end{array}\right)=\frac{4 \pi \varepsilon_{0}}{R-r}\left(\begin{array}{cc}
r R & -r R \\
-r R & R^{2}
\end{array}\right)
$$

Note that in the limits $\mathrm{R} \rightarrow \infty$ and $\mathrm{r} \rightarrow 0$ I recover the single sphere results for the diagonal elements $C_{\pi}$ and $C_{R R}$.

The easiest way to think of the full capacitance marrix is to consider each sphere as being coupled by two lumped capacitances: The first to ground at infinity, and the second to the other sphere. The equivale at circuit 
diagram is shown in Fig. A.1. The elements in the equivalent circuit are not simply the elements in the capacitance matrix. The relations are given by

$$
\begin{aligned}
& C_{1}=C_{11}+C_{21} \\
& C_{2}=-C_{12}=-C_{21} \\
& C_{3}=C_{22}+C_{12}
\end{aligned}
$$

In this case I find from the capacitance matrix that $C_{1}=0$. This makes sense, as sphere $R$ surrounds sphere $r$, and therefore screens it from the grcund at infinity: Sphere $r$ does not couple to ground. By a similar calculation I can extract $C_{2}$ and $C_{3}$. The results are that

$$
\begin{aligned}
& C_{1}=0 \\
& C_{2}=4 \pi \varepsilon_{0} \frac{r R}{R-r} \\
& C_{3}=4 \pi \varepsilon_{0} R
\end{aligned}
$$

It is amusing to see that in the limit $r \rightarrow R$, if 1 define $d=R-r$ to be the sphere spacing and $A=4 \pi r R=4 \pi R^{2}$ be the area, I find $C_{2}=\varepsilon_{0} A / d$, the result for $a$ parallel plate capacitor. In this case the result is exact, as there are no edge effects: I have connected all the edges of the parallel plate capacitor together.

Armed with the intuition from this simple example, I am ready to study the problem of the small tunnel junction and its leads. In particular, I want to look at the capacitance matrix which involves the leads on opposite sides of the tunnel junction; the geometric estimate of the capacitance of the actual tunnel junction is assumed to be accurate. The geometry of the two leads is such that analytic calculations are intractable. I therefore wrote a 


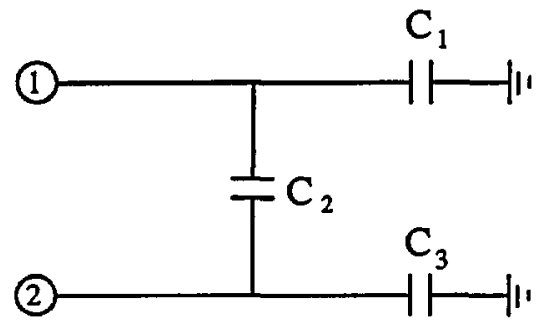

FIGURE A.1 Circuit diagran used for capacitance calculation. The metallic elements 1 and 2 have capacitances to ground $C_{1}$ and $C_{3}$, respectively, and the capacitance between 1 and 2 is $C_{2}$. 
numerical program which iteratively calculated the charge distribution on any two sheets of a conducting medium. The scheme was to break the sheets up into small squares, assume a uniform voltage and charge distribution on each square, and let the charge flow between adjacent squares until the voltages of all the squares in one sheet were roughly equal. The charge on any one square affects its own voltage through its self-capacitance, and the voltages of all the other squares through the mutual capacitive coupling. The capacitive coupling between two uniformly charged coplanar squares can be calculated analytically, as can the self-capacitance of a single square.

In other words, the real system of two metal sheets was simulated by a two sets of square charged squares, each square connected to each of its neighbors by a resistor, and the charge is allowed to flow between squares until nuss-equilibrium was achieved (see Fig. A.2). As I am interested in the full capacitance matrix, and as the two metallic sheets were geometrically symmetric, one of the sheets would be grounded by connecting each charged square of that sheet to ground (at infinity) through a resistor. This would force the voltage of that sheet to equilibrate to zero. Two elements of the capacitance matrix can then be extracted. One diagonal element is the ratio of the total charge to the average voltage on the ungrounded sheet, and the off-diagonal element is the ratio of the total charge on the grounded slieet to the average voltage on the other sheet. The three circuit elements in the equivalent circuit of Fig. A.l can then be calculated using Eq. (A.3). In all the calculations the iterations were carried out until the capacitances changed by less than $1 \%$ from one iteration to the next; this typically took about 10 iterations. 


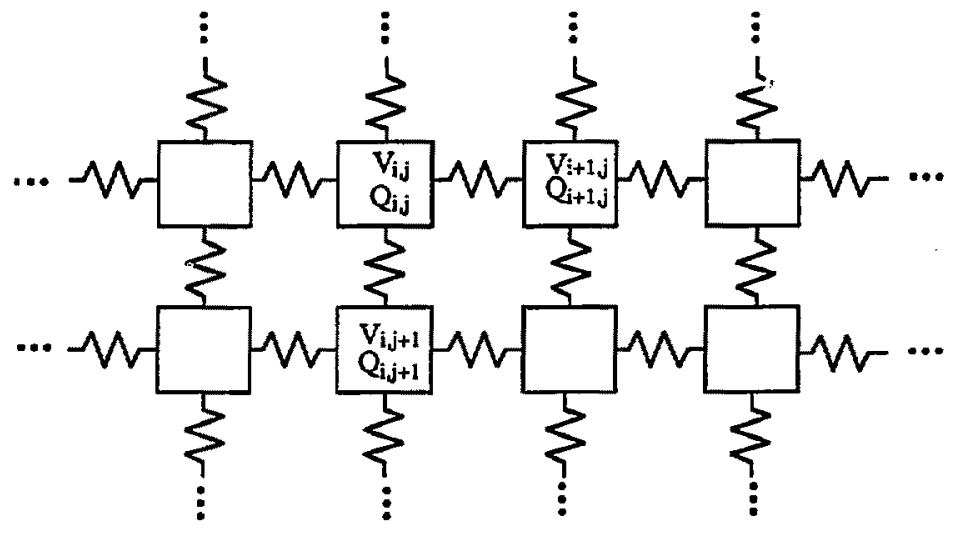

FIGURE A.2 Planar arrangement of square charged sheets connected to each other by resistors; these form a model for the capacitance calculations. Each square (i,j) is described by a voltage $V(i, j)$ and charge $Q(i, j)$; the voltage $V$ on each square is determined by the charge $Q$ on that square and, through capacitive coupling, by the charge on all the other squares. 
In Table A.1, I report the results of a number of problems given to the program. Note that if all the dimensions in the problem are scaled by the same factor, the capacitance scales by that factor (this was actually checked for two different scale factors). Most the results in the table were calculated for a scale factor of $0.1 \mu \mathrm{m}$. In all cases the two sheets were coplanar, 1 unit wide, and the narrow ends faced each other by the specified number of units (see Fig. A.3).

The most obvious points are that: 1) Both capacitances scale well with the length. 2) The diagonal capacitance $C_{11}$ (capacitance to ground) is roughly independent of sheet spacing. 3) The off-diagonal (inter-sheet) capacitance $C_{12}$ is about 10 times smaller than $C_{11}$, and is only about $70 \%$ larger when the sheet spacing is $0.1 \mu \mathrm{m}$ as opposed to $1 \mu \mathrm{m}$. 4) The capacitance matrix follows the scaling law quite well.

These calculations are for a scale factor of $0.1 \mu \mathrm{m}$. If I multiply the $360 \times 1$ calculation by a factor of 60 , I will have the capacitances for two leads $6 \mu \mathrm{m}$ wide and $2160 \mu \mathrm{m}$ long, with ends separated by $60 \mu \mathrm{m}$. This corresponds to the combinea capacitance of the two resistive leads on each side of the junction, so it should be divided by 2 to get the result for one lead. I then expect the capacitance to ground, per mm lead length, to be $3.9 \mathrm{fF} / \mathrm{mm}$. The inter-lead capacitance is calculated to be $0.35 \mathrm{fF} / \mathrm{mm}$ lead. The first figure corresponds well to the estimated result of $1-5 \mathrm{fF} / \mathrm{mm}$ for a coaxial lead model, and the latter figure indicates that I can ignore the inter-lead capacitance, at least at this level.

If I place the leads on a substrate with dielectric constant $\varepsilon_{r}$, then this value should be increased by a factor $\left(\varepsilon_{\mathrm{r}}+1\right) / 2$; for an $\mathrm{SiO}_{2}$ substrate, $\varepsilon_{\mathrm{r}}=4$ and $I$ get $C_{L}=9.8 \mathrm{fF} / \mathrm{mm}$. This was the value used for comparisons with the theory of Devoret et al. (28) (see Chapter 7). 


\begin{tabular}{ccccc}
\hline $\begin{array}{c}\text { Dimensions } \\
\text { length } \times \text { width } \\
\text { (units) }\end{array}$ & $\begin{array}{c}\text { Spacing } \\
\text { (units) }\end{array}$ & $\begin{array}{c}\text { Scale } \\
(\mu \mathrm{m})\end{array}$ & $\begin{array}{c}\mathrm{C}_{11} \\
\text { (fF) }\end{array}$ & $\begin{array}{c}\mathrm{C}_{12} \\
(\mathrm{fF})\end{array}$ \\
\hline $20 \times 1$ & 10 & 1.0 & 0.257 & -0.0227 \\
$20 \times 1$ & 10 & 0.1 & 0.0257 & -0.00228 \\
$40 \times 1$ & 10 & 0.1 & 0.0444 & -0.00451 \\
$80 \times 1$ & 10 & 0.1 & 0.0782 & -0.00817 \\
$180 \times 1$ & 10 & 0.1 & 0.154 & -0.0149 \\
$360 \times 1$ & 10 & 0.1 & 0.279 & -0.0255 \\
$80 \times 1$ & 100000 & 0.1 & 0.0774 & $-0.99 \times 10-5$ \\
$40 \times 1$ & 5 & 0.1 & 0.0448 & -0.00511 \\
$40 \times 1$ & 3 & 0.1 & 0.0451 & -0.00571 \\
$40 \times 1$ & 2 & 0.1 & 0.0453 & -0.00610 \\
$40 \times 1$ & 1 & 0.1 & 0.0458 & -0.00691 \\
\hline
\end{tabular}

TABLE A.1 This table shows the result of iterative calculations of the capacitance of two metal sheets, with layout and dimensions as shown in Fig. A.3. The first column gives the length and width of the sheets, the second column the spacing between the ends of the sheets; the scale is the actual dimension of one length unit, and the final two columns give the selfcapacitance and inter-line spacing of the given geometry. 


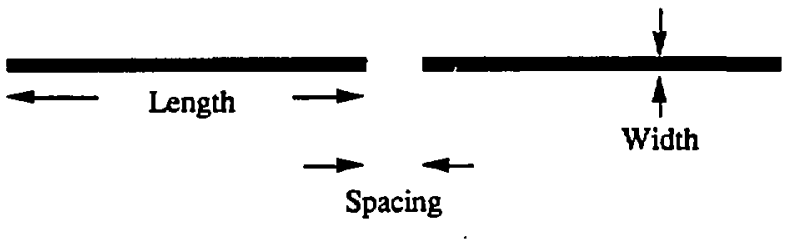

FIGURE A.3 Terms used for various dimensions specifying the geometry for the capacitance calculations of the resistive leads. Each lead is assumed infunitesimally thick and in the same plane as the opposing lead. 


\section{Appendix B - Resistive Transmission Line}

In this appendix I sketch out the calculation of the impedance of an infinite length and a finite length resistive transmission line, whose differential element is shown in Fig. B.1. The resistance, inductance and capacitance per unit length are given by $R, L$, and $C$. The voltage $\mathrm{V}(\mathrm{x}, \mathrm{t})$ and

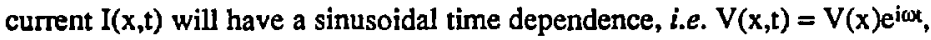
and the spatial equations are

$$
\frac{\mathrm{d}^{2} \mathrm{~V}(\mathrm{x})}{\mathrm{dx^{2 }}}=(R+i \omega L) i \omega C \mathrm{~V}(\mathrm{x})
$$

and

$$
\frac{\mathrm{dI}(\mathrm{x})}{\mathrm{dx}}=-i \omega C \mathrm{~V}(\mathrm{x})
$$

With the assumption $V(x)=V_{0} \exp (-i k x-\alpha x)$ and $I(x)=I_{0} \exp (-i k x-\alpha x)$, I get the two equations

$$
2 \mathrm{k} \alpha=\omega R C
$$

and

$$
k^{2}-\alpha^{2}=\omega^{2} L C
$$

These equations may be solved for the characteristic impedance $Z_{0}=V_{0} / I_{0}$ $=(i \mathrm{k}+\alpha) /(i \omega C)$ and for the phase velocity $\mathrm{v}=\omega / \mathrm{k}$. I define the compound quantity $\gamma=\sqrt{\omega R} \bar{C} / 2$, and in the limit of frequencies $\omega$ where the 


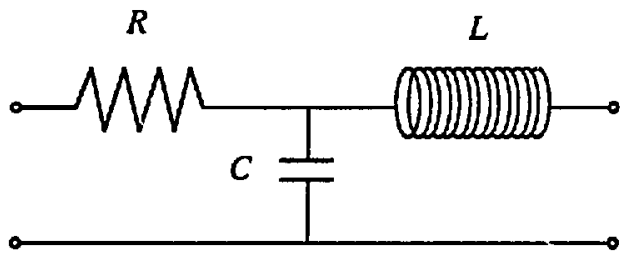

FIGURE B.1 A single element of the resistive transmission line for the resistive leads. The line has resistance per unit length $R$, capacitance $C$, and inductance $L$, and an infinitesimal length $d x$ of the line is shown here. 
inductance $L$ does not contribute significantly to the impedance (i.e. $\omega L \ll$ $R$ ), I find that $\mathrm{k}=\alpha=\gamma=\sqrt{\omega R C / 2}$, the characteristic impedance is $\mathrm{Z}_{0}=$ $(R / 2 \gamma)(1-i)$, and the phase velocity is $\mathrm{v}=\sqrt{2 \omega / R C}$.

I now apply these results to the problem of a terminated resistive transmission line of length $\Lambda$. The time dependence of the voltage and current is the same, but the spatiol dependence is now given by

$$
V(x)=V_{1} \exp (-i k x-\alpha x)+V_{2} \exp (i k x+\alpha x)
$$

and

$$
I(x)=I_{1} \exp (-i k x-\alpha x)+I_{2} \exp (i k x+\alpha x)
$$

where $k$ and $\alpha$ satisfy Eqs. (B.3) and (B.4), and the subscript 1 corresponds to a left-going wave and the subscript 2 to a right-going wave.

The boundary conditions for a short at $x=\Lambda$ are $V(0)=V_{0}$, and $V(\Lambda)=0$. I use a short at $x=\Lambda$ because the large stray capacitance and the low-impedance coaxial line starting at that point are nearly indistinguishable from a short, and using a short makes the math easier. Solving for $V_{i}$ and $I_{j}$ is straightforward, and I can extract the impedance $Z$ of the line at $x=0$ in terms of the characteristic impedance $\mathrm{Z}_{0}$,

$$
\begin{aligned}
Z & =Z_{0}\left(\frac{1-\exp (-2}{1+\exp (-2} \frac{\Lambda(i k+\alpha))}{(i k+\alpha))}\right) \\
& \left.=Z_{0} \operatorname{coth}(\Lambda(i k+\alpha))\right)
\end{aligned}
$$


In the low frequency limit, this reduces to $Z=Z_{0} \operatorname{coth}(\Lambda \gamma(1+i))$. For a very short line $\Lambda \ll \gamma, I$ find $Z=R \Lambda=R$, and for a very long line $I$ have $Z=$ $\sqrt{R / 2 \omega C}(1-i)$. It is important to note that in the long line limit, which is the one I am interested in here, the impedance falls as the inverse root of the frequency, and that it has an imaginary part as large as the real part. The cutoff between the long and short line limits is at $\omega(R \Lambda)(C \Lambda)=\omega R C=1$. I will want an exact expression for the impedance $Z$ as a function of frequency; this expression is quite messy, but useful, so it is reproduced here. I define the following quantities:

$$
\begin{aligned}
k_{0} & =\sqrt{\omega^{2} L C} \\
G & =2 \gamma \Lambda \\
& =\sqrt{2 \omega R C} \\
f^{2} & =\frac{k_{0}{ }^{2}}{2 \gamma^{2}}+\sqrt{1+k_{0} / 4 \gamma^{4}} .
\end{aligned}
$$

I then have the real and imaginary parts of $Z$ given by

$$
\operatorname{Re}\left(\frac{Z}{R}\right)=\frac{1}{D}\left(\frac{f}{G}\left(1-e^{-2 G / I}\right)+\frac{2}{f G} \sin (f G) e^{-G / E}\right)
$$

and

$$
\operatorname{Im}\left(\frac{Z}{R}\right)=\frac{1}{D}\left(-\frac{1}{f G}\left(1-e^{-2 G / f}\right)+\frac{2 f}{G} \sin (f G) e-G / f\right)
$$


where the denominator $D$ is given by

$$
D=1+e^{-2 G / f}+2 e^{-G / t} \cos (f G)
$$

In Fig. B.2 I have plotted the real and imaginary parts of the impedance as a function of frequency; note the sharp cutoff at $\omega R C=1$, where the real part of $Z$ falls precipitously and the imaginary part displays a resonarice. Note also the close resemblance of this plot to those of the real and imaginary parts of the dielectric response function $\varepsilon(\omega)$ : both $\varepsilon$ and $Z$ satisfy the KramersKronig causality relations. 


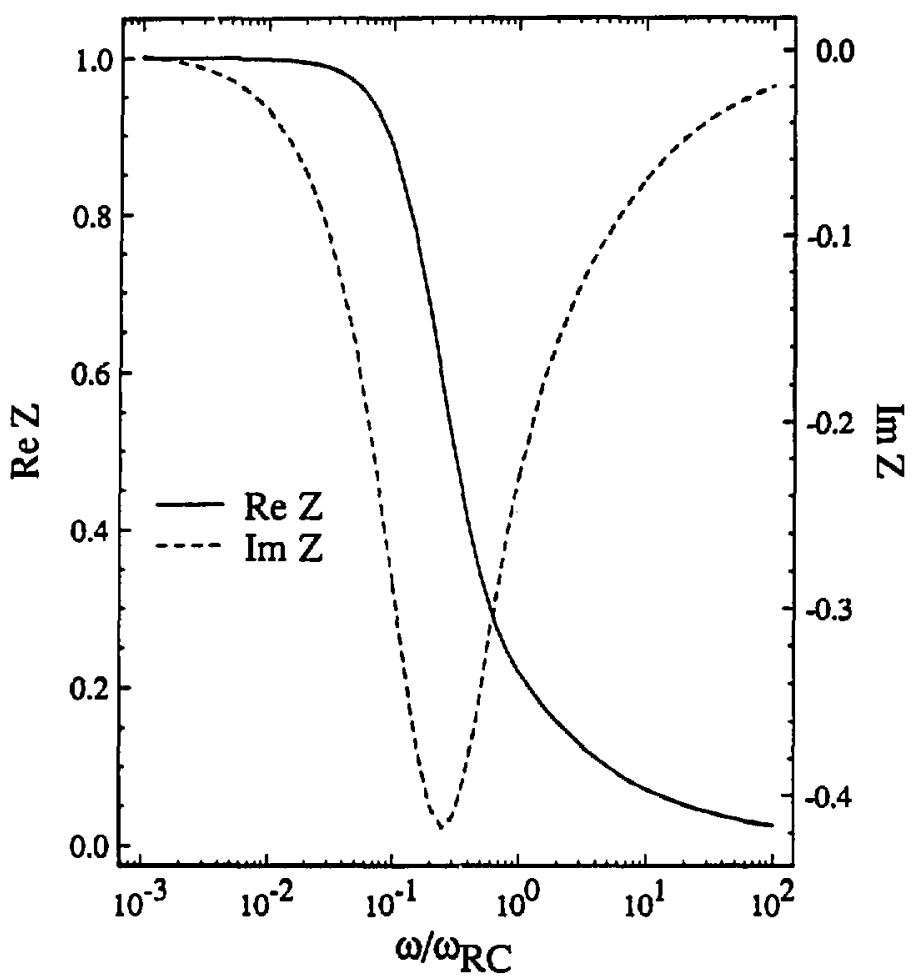

FIGURE B.2 Real and imaginary parts of the impedance function $Z(\omega)$ as a function of frequency $\omega$; the real part is the solid line and the imaginary part the dashed line. This plot applies to both the $\mathrm{CuAu}$ and the NiCr leads. 


\section{Appendix C - Electronics}

Some of the measurements described here required somewhat specialized electronics boxes to deal with the large resistances encountered with the resistive leads and junctions connected to them. In particular, I built two FET-input amplifier boxes, one as a current source and the other as a low-noise pre-amplifier. I describe each box here, for reference purposes.

\section{Current Source}

The model current source circuit is shown in Fig. C.1. As I was concemed about the output impedance of the current source, as well as its noise characteristics, I carried out some calculations to estimate each of these. The standard first-order model is shown in Fig. C.2 (see P. Horowitz and W. Hill, The Art of Electronics, Cambridge University Press, New York (1980), p. 129).

The current I through the load resistor $R_{L}$ is given by

$$
I=\left(\frac{\frac{G}{R_{L}}-(G+1) \frac{R_{B}}{R_{L} R_{i n}}}{1+(G+1) R_{B} / R_{L}}\right) V_{\text {in }} .
$$

where $R_{\text {in }}$ is the input resistance of the circuit seen by $V_{\text {in }}$ (see below), $R_{B}$ is the bias resistor, and $G$ is the open-loop gain of the op-amp. In the limit $G$ $\gg 1$ and $R_{i n} \gg R_{L}$, the current is given by

$$
I=\frac{V_{\text {in }}}{R_{B}} \frac{1}{I+R_{L} / G R_{B}}
$$




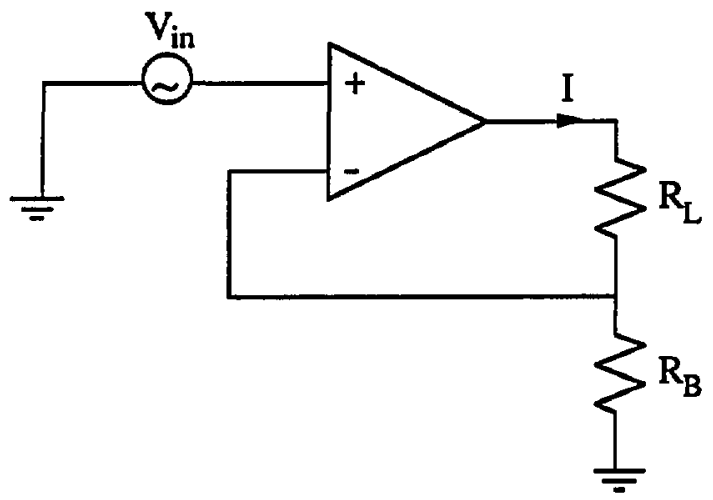

FIGURE C.1 Circuit used for FET amplifier current source. The load is $R_{i}$, the bias resistor $R_{B}$, and the current source is controlled by the voltage source $\mathrm{V}_{\mathrm{in}}$. 
so a good current source requires $G_{B} \gg R_{L}$. The input impedance $R_{\text {in }}$ of the circuit is given by

$$
R_{\text {in }}=\frac{R}{1+R_{B} / R_{L}}\left(\left(1+(G+1) \frac{R_{B}}{R_{L}}\right)+\frac{R_{B}}{R}\right) \text {. }
$$

where $R$ is the op-amp open-loop input impedance (for an FET op-amp $R=10^{12} \Omega$ ). In the limit $G \gg 1, R \gg R_{B}$ I find the result

$$
R_{\text {in }}=G R \frac{1}{1+R_{L} / R_{B}}
$$

so the condition on the current source $R_{\text {in }} \gg R_{L}$ is met by requiring that $G R$ $\gg R_{L}$; this is easily met. Typical FET op-amps have gains $G=10^{4}$, and the circuit only works as a current source in the limit $G R_{B} \gg k_{L}$, so the load resistor $R_{L}$ cannot be much larger than 100 times the bias resistance $R_{B}$. For a load $R_{L}$ of $1 \mathrm{G} \Omega$, and with gain $G=10^{4}$, the bias resistor should be at loast $10 \mathrm{M} \Omega$; again, this is easily accomplished.

The output impedance $R_{01}$ seen by the load is given by

$$
R_{\text {out }}=(1+G) R_{B},
$$

which as expected requires $G R_{B} \gg R_{L}$ for a current source.

The principal sources of noise in this circuit are the current noise $\mathbf{i}_{\mathbf{n}}$ and the voltage noise $e_{n}$ from the FET op-amp, and the Johnson noise $i_{R}$ in the bias resistor $R_{B}$, which is at room temperature. The load $R_{L}$ is assunied to be at $20 \mathrm{mK}$, and its current noise is theref $A$ ignored. The current noise $I_{n}$ through the load resistor $R_{L}$ is then given by 


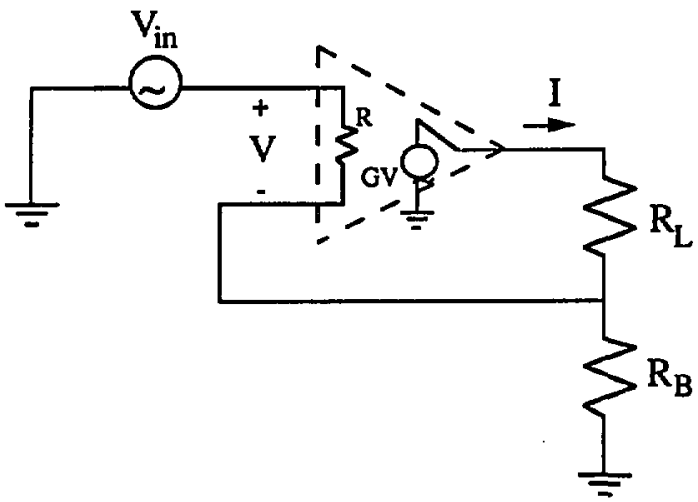

FIGURE C. 2 Current source circuit showing model for FET operational amplifier. The op-amp has input resistance $R$ and gain $G$. 


$$
I_{n}^{2}=\left(e_{n} / R_{B}\right)^{2}+i_{n}^{2}+i_{R}^{2}
$$

where $i_{R}=\sqrt{4 k_{B} T / R_{B}}$, and $\mathrm{T}=300 \mathrm{~K}$. This noise goes down monotonically with $R_{B}$, with a knee at $R_{B}=e_{n} / i_{n}$; for $R_{B}$ larger than this value, the noise is primarily due to the FET current noise source.

The actual circuit built is shown in Fig. C.3. The bias resistor $R_{B}$ could be 1,10 or $50 \mathrm{M} \Omega$. The gain $\mathrm{G}$ was about $10^{4}$ and the input impedance was $R$ $=10^{12} \Omega$; this circuit could comfortably handle loads $R_{L}$ up to about 5 to 10 $G \Omega$. With a $10 \mathrm{G} \Omega$ load, however, only about $1 \mathrm{nA}$ maximum bias current is allowed, due to the voltage supply limits. The noise from this circuit was measured to be limited at higher frequencies $(>10 \mathrm{~Hz}$ ) by the FET current noise of $15 \mathrm{fA} / \sqrt{\mathrm{Hz}}$, and at low frequencies was dominated by the $1 / \mathrm{f}$ voltage noise of the FET, which at $1 \mathrm{~Hz}$ was $75 \mathrm{nV} / \sqrt{\mathrm{Hz}}$. With a $50 \mathrm{M} \Omega$ bias resistor, the total current noise through the load at $1 \mathrm{~Hz}$ was $24 \mathrm{fA} / \sqrt{\mathrm{Hz}}$.

It is useful to note that by switching the load and bias resistors, this circuit becomes a voltage source, with extremely low output impedance.

\section{Differential Preamplifier}

The high-impedance differential preamplifier I used, which is a standard differential amplifier configuration, is shown in Fig. C.4. Each LF 356 FET op-amp has a gain of 19.5, and the OP-27 difference follower has unity gain. All the resistors except the $1.1 \mathrm{k} \Omega$ resistor were carefully matched to within $0.1 \%$. The complete amplifier has a gain of 19.5 , and all the noise is from the voltage noise of the FET op-amps; above $100 \mathrm{~Hz}$ this is $18 \mathrm{nV} / \sqrt{\mathrm{Hz}}$, and below $10 \mathrm{~Hz}$ the noise is $1 / \mathrm{f}$; at $1 \mathrm{~Hz}$ the noise was measured to be $150 \mathrm{nV} / \sqrt{\mathrm{Hz}}$. The amplifier had $30 \mathrm{ppm}$ common-mode rejection at 100 
$\mathrm{Hz}$, and had a $3 \mathrm{~dB}$ rolloff in gain at $550 \mathrm{kHz}$. The input impedance of the amplifier is determined by the FET op-amps, and is given on the data sheets as $10^{12} \Omega$. 


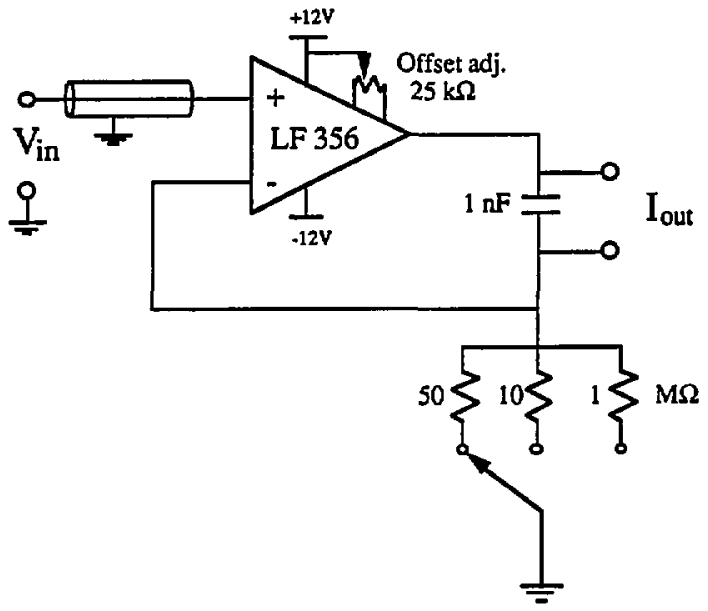

FIGURE C.3 Circuit schematic for current source. The input voltage $V_{\text {in }}$ is applied from an external source, and the bias resistor is selectable. A $1 \mathrm{nF}$ output capacitor is used to reduce high-frequency noise. 


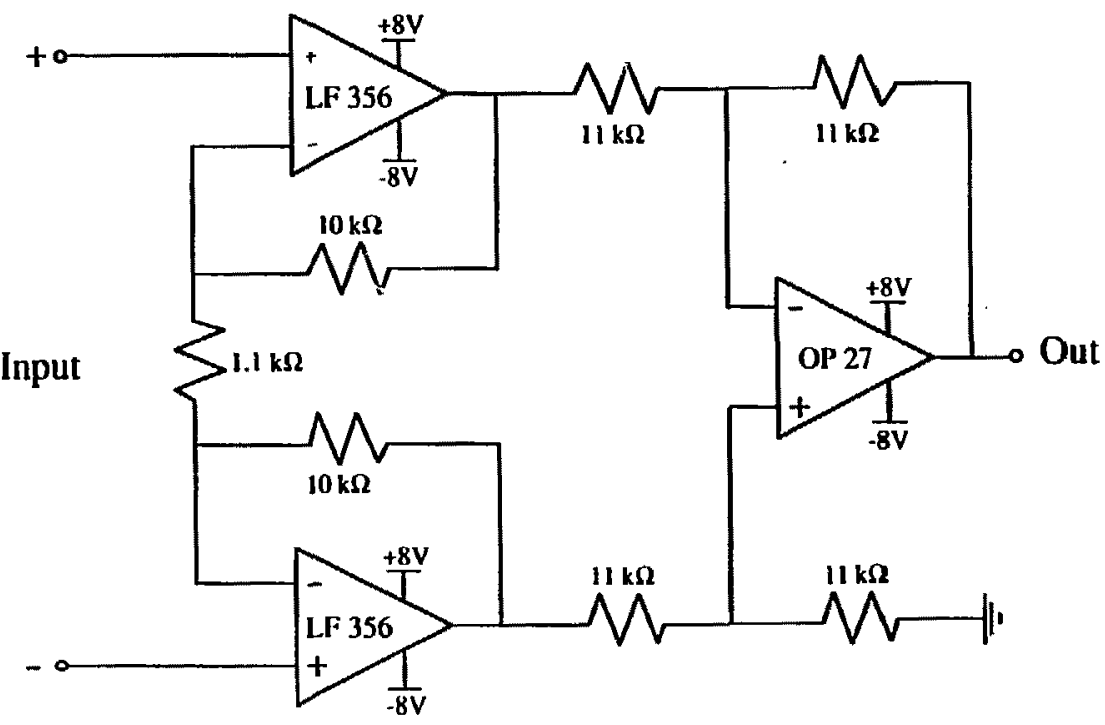

FIGURE C.4 Circuit schematic for differential amplifier. The two input op-amps have high input resistance, and the resistors are all carefully matched to get good common-mode rejection. 


\section{Appendix D - Small Junction I-V Characteristic}

In this appendix I will discuss some of the implications of the approximate current bias for the small junctions. I begin by sketching out the argument for the low bias current limit of the normal metal tunnel junction I$V$, i.e. the region of the current-voltage characteristic where I depends parabolically on V; this applies to the truly current-biased situation.

In the voltage-biased case at zero temperature, the I-V characteristic may be calculated from the semiconductor model of electron tunneling (47). In this model the current is given by

$$
I=\frac{2 \pi}{\hbar} \int_{-\infty}^{\infty} N_{1} N_{2}|M|^{2}\left(f_{1}(E)\left[1-f_{2}(E+e V)\right]-\left[1-f_{1}(E)\right] f_{2}(E+e V)\right) d E
$$

where $N_{1}$ and $N_{2}$ are the density of states in electrodes 1 and $2,|M|^{2}$ is the tunneling matrix element, and $f_{1}$ and $f_{2}$ are the Fermi distribution functions.

Assuming that $\mathrm{N}_{\mathrm{i}}$ and $\mathrm{IM} / 2$ are independent of energy, the integral may be done simply, with the linear result

$$
\begin{aligned}
I & =\frac{2 \pi}{\hbar} N_{1} N_{2} I T / 2 \mathrm{eV} \\
& =V / R_{J}
\end{aligned}
$$

where $R_{J}$ is the junction tunnel resistance; this result is independent of temperature. If I now allow the voltage of electrode 2 to change with respect to electrode 1, due to the Coulomb charging of the finite inter-electrode capacitance $\mathrm{C}$, I must replace $\mathrm{E}+\mathrm{eV}$ with $\mathrm{E}+\mathrm{eV}-\mathrm{e}^{2 / 2 C}$, and I get the results 


$$
\begin{array}{rlr}
\mathrm{I} & =0 & \left(0<\mathrm{eV}<\mathrm{e}^{2} / 2 \mathrm{C}\right) \\
\mathrm{I} & =\mathrm{N}_{1} \mathrm{~N}_{2} \mathrm{TT}^{2}\left(\mathrm{eV}-\mathrm{e}^{2} / 2 \mathrm{C}\right) & \left(\mathrm{eV}>\mathrm{e}^{2} / 2 \mathrm{C}\right) \\
& =(\mathrm{V}-\mathrm{e} / 2 \mathrm{C}) / \mathrm{R}_{\mathrm{J}} &
\end{array}
$$

which is the voltage-biased I-V characteristic; in this case I am restricting the discussion to $T=0$. The turneling rate $\Gamma$ is given by $\Gamma(V)=1(V) / e$.

In a current-bias situation, the voltage $V$ increases linearly with time; once it exceeds $\mathrm{e} / 2 \mathrm{C}$, there is a probability that an electron will tunnel. The junction follows a sawtooth-shaped charging and discharging sequence, where the discharging events are described by a stochastic equation; the current-voltage characteristic can be calculated using the voltage-biased tunneling rate calculated above. No tunneling can occur during the charging sequence until $\mathrm{V}$ exceeds $\mathrm{e} / 2 \mathrm{C}$; if I set time $\mathrm{t}=0$ at the moment $\mathrm{V}$ exceeds $e / 2 C$, then $V(t)=e / 2 C+I t / C$. Given the probability $P(t)$ that an electron has not tunneled at time $D 0$, the probability $\mathrm{dP}$ that an electron will not tunnel in the next interval dt satisfies

$$
\begin{aligned}
\mathrm{dP} & =-\Gamma(\mathrm{V}) \mathrm{P}(\mathrm{t}) \mathrm{dt} \\
& =-((\mathrm{V}-\mathrm{e} / 2 \mathrm{C}) / \mathrm{eR}) \mathrm{P}(\mathrm{t}) \mathrm{dt}
\end{aligned}
$$

Integrating Eq. (D.5) gives

$$
P(t)=N \exp \left(-I t^{2} / 2 e R C\right)
$$


where $\mathrm{N}$ is the normalization to get unit probability at infinite time, and is given by $\mathrm{N}^{2}=\pi \mathrm{I} / 2 \mathrm{eRC}$. The mean value of voltage at which tunneling occurs is then

$$
V=e / 2 C+\int_{0}^{\infty}(I t / C) P(t) d t,
$$

and the mean voltage after several oscillations, which gives the I-V characteristic, is then

$$
\begin{aligned}
V & =\int_{0}^{\infty}(I t / C) P(t) d t \\
& =N(I / C)(e R C / I) \\
& =\sqrt{\pi e R} \overline{I / 2 C}
\end{aligned}
$$$$
\left(\mathrm{eV} \ll \mathrm{e}^{2} / 2 \mathrm{C}\right)
$$

which gives a parabolic small-current I-V. At large currents the I-V will be linear, given by

$$
\mathrm{I}=(\mathrm{V}-\mathrm{e} / 2 \mathrm{C}) / \mathrm{RJ}_{\mathrm{J}} \quad\left(\mathrm{eV}>\mathrm{e}^{2} / 2 \mathrm{C}\right)
$$

which is indistinguishable from the voltage-biased limit. The low-current limit of the two biasing schemes are however very different.

A plot comparing the zero-temperature voltage-biased and the numerically calculated cument-biased current-voltage characteristic is 


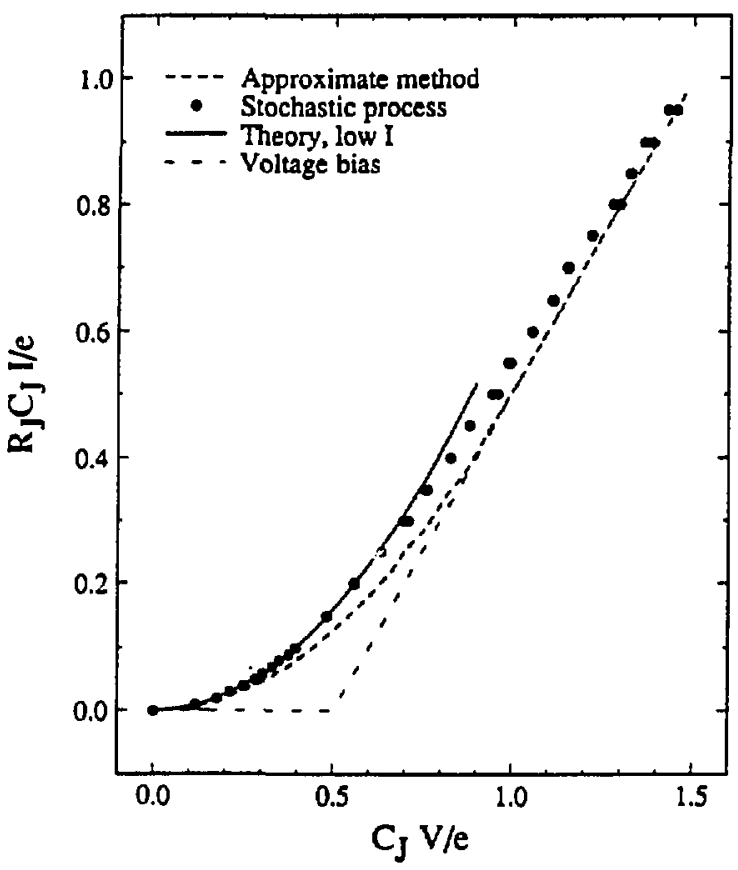

FIGURE D.1 Current-voltage characteristic numerically calculated from charging-discharging sequence of a junction at $T=0$. Also shown is the voliage-biased characteristic and the low-current bias part of the I-V, calculated from Eq. (D.8). 
shown in Fig. D.1. Also shown is the low bias current limit, calculated from Eq. (D.8).

The calculation sketched out above is for a true current-bias, in other words a junction connected to an ideal current source with no intervening circuit. In the actual experiments discussed in this thesis, the circuit is better approximated by that shown in Fig. D.2. The stray capacitance $\mathrm{C}_{\text {stray, }}$, assumed much larger than the junction capacitance $C_{j}$, acts as a voltage source, and for bias currents $I_{b i a s}$ such that $e / I_{b i a s} \gg R_{L} C_{J}$ the junction is effectively voltage-biased. However, for higher bias currents such that $\mathrm{e} / \mathrm{I}_{\text {bias }} \ll<R_{\mathrm{L}} \mathrm{C}_{\mathrm{J}}$, the lead resistor produces a current-bias situation. The charging-discharging sequences for either bias limit is sketched in Fig. D.3. The cursent-voltage characteristic in this situation may be calculated numerically, using the discharging probability as a function of voltage and using the circuit of Fig. D.2 to calculate the voltage as a function of time. However, I developed an approximate way to calculate the current-voltage characteristic of such a device, which is simple and involves far less computation time to produce a current-voltage characteristic.

The basic approximation of this technique is to assume that the time between electron tunneling events $\Delta t=e / I_{\text {bias }}$ is fixed for all events. In the actual system the average time between events is given by $\Delta t$, but the actual times vary about this average. Given this single assumption, the voltage $V(t)$ from time $t$ to time $t+\Delta t$ can be calculated by solving the circuit equations for the circuit of Fig. D.2, and then calculating the current Ibias given by

$$
I_{\text {bias }}=\frac{1}{\Delta t} \int_{t}^{t+\Delta t} \Gamma(V(t)) d t
$$




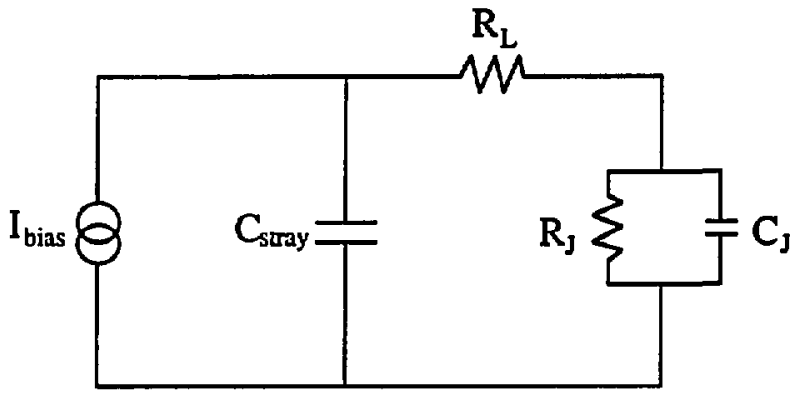

FIGURE D.2 Approximate circuit used to calculate current-voltage chäracteristic of a small junction with tunnel resistance $\mathrm{RJ}_{\mathrm{J}}$ and capacitance $C_{J}$; the lead resistance $R_{L}$ and stray capacitance $C_{\text {sray }} \gg C_{J}$ affect the bias description, as discussed in the text. 
Appendix D - Small Junction I-V Characteristics

155

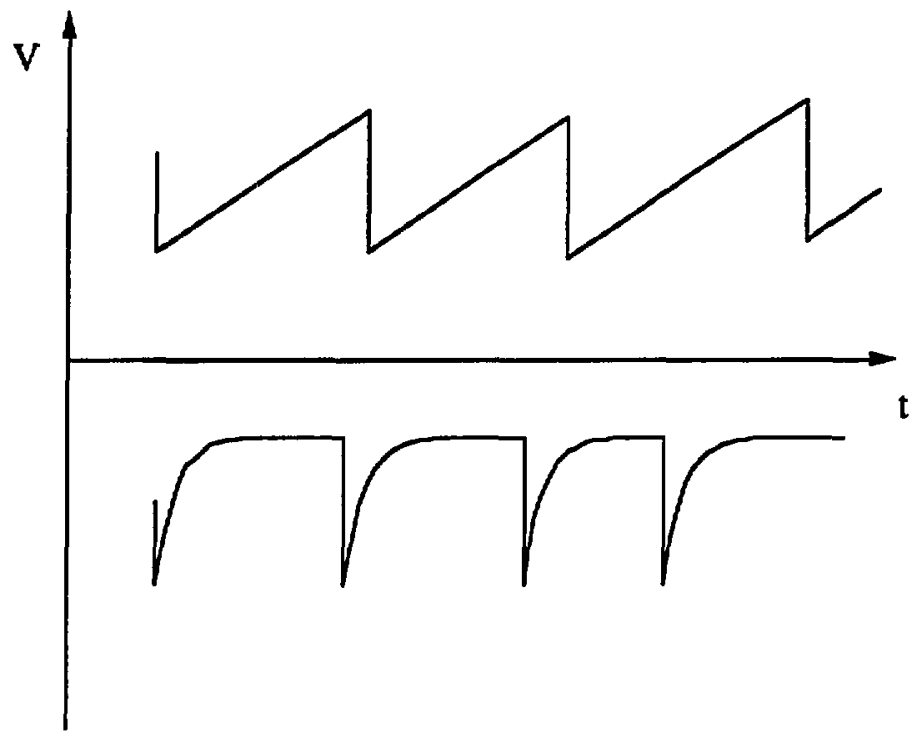

FIGURE D.3 The upper trace shows the charging-discharging sequence in the limit $e / I_{\text {bias }} \gg R_{L} C_{j}$ and the lower trace is the opposite limit $e / I_{b i a s} \ll$ $\mathrm{R}_{\mathrm{L}} \mathrm{C}_{\mathrm{J}}$. 
The average voltage measured for this value of bias current is given by

$$
<V>=\frac{1}{\Delta t} \int_{t}^{t+\Delta t} V(t) d t
$$

The I-V characteristic may be calculated analytically for the true current bias case (i.e. for $\mathrm{R}_{\mathrm{L}}=\infty$ ); a comparison of this approximate calculation with a numerical simulation is shown in Fig. D.4; the approximate method works quite well, with the largest errors of about 10\% near the knee of the Coulomb blockade. A comparison of the differential conductance, calculated using the approximate method and comparing to the numerical results, is shown in Fig. D.5; again, the agreement is fairly good over the full range of bias currents.

If I now allow the lead resistance $R_{L}$ to become finite, the results become quite interesting; in Fig. D.6 I show the current-voltage chaiacteristics resulting from a circuit with junction parameters $R_{J}=10 \mathrm{k} \Omega$, $C_{J}=1 \mathrm{fF}, C_{\text {stray }}=100 \mathrm{fF}$ and a range of lead resistances $R_{L}$ from $1 \mathrm{k} \Omega$ to 10 MS; note that the fluctuations in this circuit, causing smearing of the Coulomb blockade, have been ignored for simplicity. For the lowest lead resistance the current-biased device is clearly indistinguishable from the voltage-biased Coulomb blockade; as the lead resistance $R_{\mathbf{L}}$ is increased the I$V$ characteristic approaches the classic current-bias limit, but at very low currents (corresponding to very large $\Delta t=e / I_{b i a s}$ ) the $I-V$ is that of the voltage-biased device. The resulting feature at low bias current is remarkably similar to that predicted for the superconducting small junction, the so-called "Bloch nose" (19).

The approximate calculation using the circuit of Fig. D.2 is acceptable when using fluctuation-induced tunneling rate $\langle\Gamma(V)>$ given by the quantum 


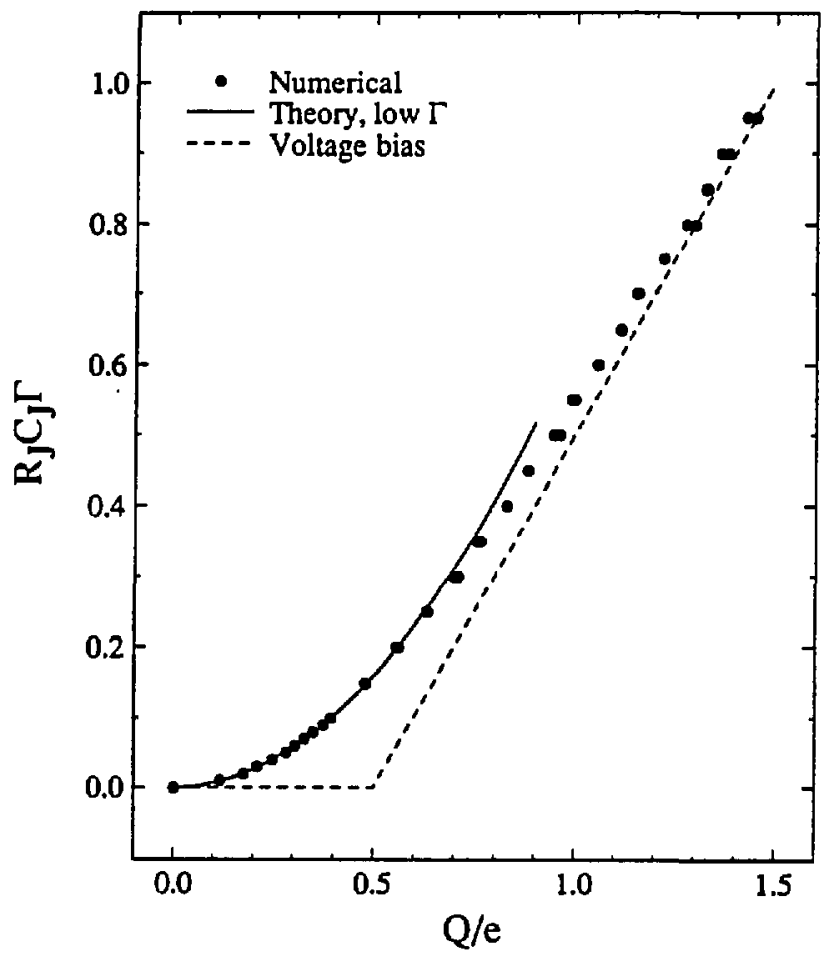

FIGURE D.4 Comparison of the current-voltage characteristic obtained from stochastic charging-discharging simulation with that obtained from the approximate regular-tunneling method. Also shown in the zero-temperature voltage biased Coulomb blockade. 


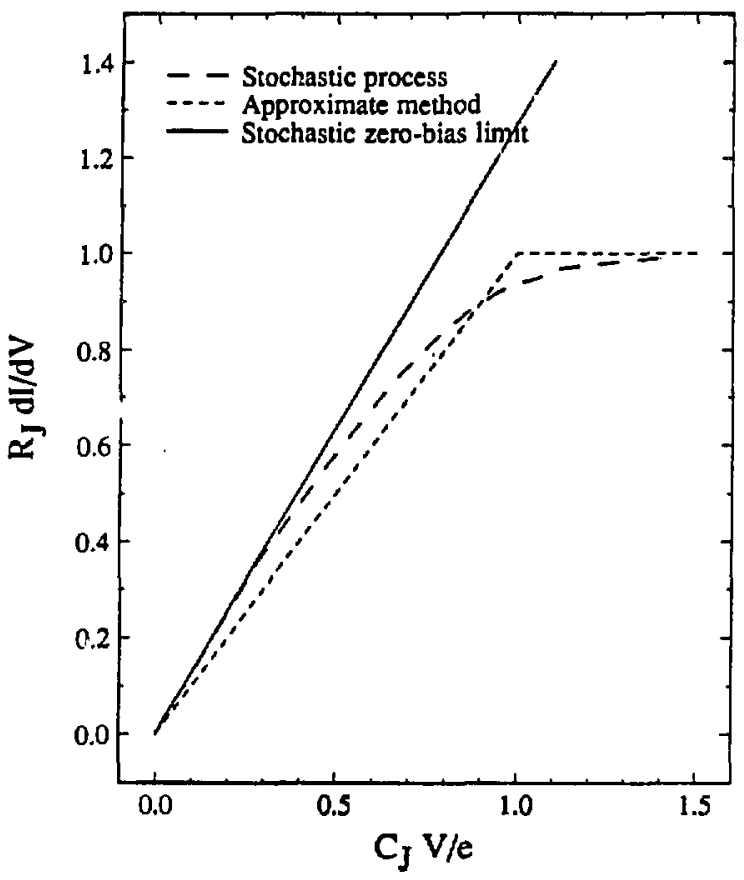

FIGURE D.5 Comparison of the differential conductance calculated from the stochastic charging-discharging sequence with that calculated using the approximate regular-nunneling method. Also shown is the zero-temperature Coulomb blockade result and the zero-bias limit of the stochastic nunneling conductance. 


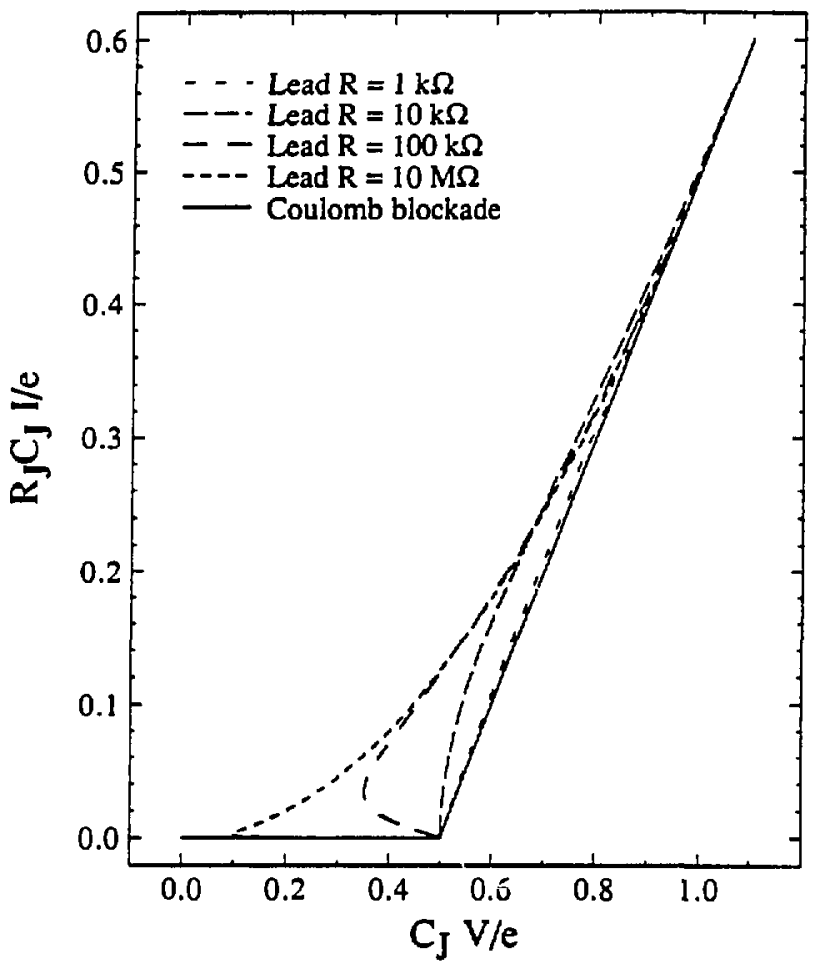

FIGURE D.6 Result of calculating the zero-temperature I-V characteristic of a small junction connected to the circuit of Fig. D.2. The stray capacitance is chosen to have the value $C_{\text {stray }}=100 \mathrm{fF}$, the junction parameters are $C_{j}=1$ $\mathrm{fF}$ and $\mathrm{RJ}=10 \mathrm{k} \Omega$, and the lead resistance is varied from $1 \mathrm{k} \Omega$ to $10 \mathrm{M} \Omega$. 
Langevin theory. When using the phase correlation theory, however, it is more reasonable to use the full impedance $Z(\omega)$ to calculate the timedependant voltage $V(t)$. For those calculations I replaced the circuit of Fig. D. 2 by that of Fig. D.7, where the current source $I_{J}(t)$ gives the regular tunneling of electrons across the junction: $\mathrm{IJ}_{\mathrm{J}}(\mathrm{t})$ is given by the sum of $\delta$ functions with amplitude $-e$ and spacing $\Delta t=e / I_{b i a s}$ in time. The voltage $V(\omega)=Z_{\mathrm{t}}(\omega) I_{\mathrm{J}}(\omega)$ is calculated from the parallel impedance of the junction capacitance and the RLC transmission line, $Z_{\mathfrak{t}}^{-1}(\omega)=i \omega C+Z^{-1}(\omega)$. Fourier transforming to get $V(t)$ then allows one to calculate the $\mathrm{I}-\mathrm{V}$ characteristic as in Eqs. (D.10) and (D.11). This was the method used to compare the results of the phase correlation theory of Devoret $e t$ al. with the experimental results. 


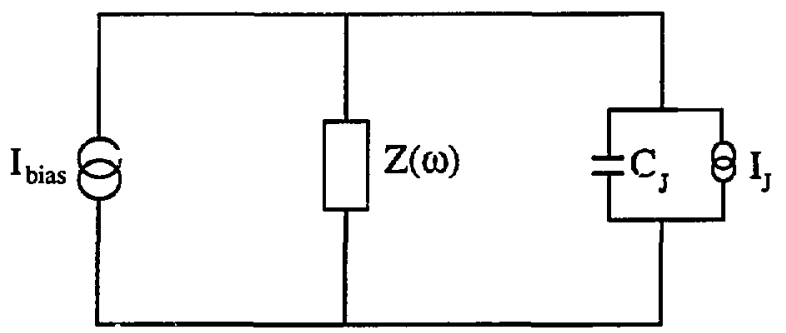

FIGURE D.7 Circuit used to calculate the I-V characteristic using the more general impedance $Z(\omega)$ in parallel with the junction capacitance $C_{J}$. The current source $I_{J}(t)$ simulates the regular tunneling of electrons across the junction. 


\section{REFERENCES}

1. A. J. Leggett, Prog. Theor. Phys. (Suppl.) 69, 80 (1980).

2. Y. M. Ivanchenko, L. A. Zil'berman, ?h. Eksp. Teor. Fiz. 55, 2305 (1968).

3. A. O. Caldeira, A. J. Leggett, Ann. Phys. (N.Y.) 149, 374 (1983).

4. A. I. Larkin, Y. N. Ovchinnikov, Pis'ma Zh. Eksp. Teor. Fiz. 37, 322 (1983).

5. H. Grabert, U. Weiss, P. Hänggi, Phys. Rev. Lett 52, 2193 (1984).

6. H. Grabert, U. Weiss, Phys. Rev. Lett. 53, 1787 (1984).

7. W. Zwerger, Phys. Rev. A 31, 1745 (1985).

8. P. S. Riseborough, P. Hänggi, E. Friedkin, Phys. Rev. A 32, 489 (1985).

9. L. D. Jackel, W. W. Webb, J. E. Lukens, S. S. Pei, Phys. Rev. B 9, 115 (1974).

10. T. A. Fulton, L. N. Dunkleberger, Phys. Rev. B 9, 4760 (1974).

11. W. den Boer, R. de Bruyn Ouboter, Physica 98B, 185 (1980).

12. R. F. Voss, R. A. Webb, Phys. Rev. Lett 47, 265 (1981).

13. L. D. Jackel, et al., Phys. Rev. Lett 47, 69 (1981).

14. S. Washbum, R. A. Webb, R. F. Voss, S. M. Faris, Phys. Rev Letf. 54, 2712 (1985).

15. J. M. Martinis, M. H. Devoret, J. Clarke, Phys. Rev. Lett. 55, 1543 (1985).

16. M. H. Devoret, J. M. Martinis, J. Clarke, Phys. Rev. Lett. 55, 1908 (1985). 
17. D. B. Schwartz, B. Sen, C. N. Archie, J. E. Lukens, Phys. Rev. Lett. 55, 1547 (1985).

18. A. D. Zaiken, S. V. Panyukov, JETP Lett. 43, 670 (1986).

19. K. K. Likharev, A. B. Zorin, J. Low Temp. Phys. 59, 347 (1985).

20. D. V. Averin, K. K. Likharev, J. Low Temp. Phys. 62, 345 (1986).

21. T. A. Fulton, G. J. Dolan, Phys. Rev. Lett. 59, 109 (1987).

22. M. Iansiti, A. T. Johnson, C. J. Lobb, M. Tinkham, Phys. Rev. Lett. 60, 2414 (1988).

23. L. S. Kuzmin, P. Delsing, T. Claeson, K. K. Likharev, Phys. Rev. Lett. 62, 2539 (1989).

24. P. Delsing, K. K. Likharev, L. S. Kuzmin, T. Claeson, Phys. Rev. Lett. 63, 1180 (1989).

25. P. Delsing, K. K. Likharev, L. S. Kuzmin, T. Claeson, Phys. Rev. Lett. 63, 1861 (1989).

26. L. J. Geerligs, V. F. Anderegg, C. A. v. d. Jeugd, J. Romijn, J. E. Mooij, Europhys. Lett. 10, 79 (1989).

27. L. J. Geerligs, et al., Phys. Rev. Lett. 64, 2691 (1990).

28. M. H. Devoret, et al., Phys. Rev. Lett. 64, 182 (1990).

29. W. C. Stewart, Appl, Phys. Lett. 12, 277 (1968).

30. D. E. McCumber, J. Appl. Phys. 39, 2503 (1968).

31. H. B. Callen, T. A. Welton, Phys. Rev. 83, 34 (1951).

32. H. A. Kramers, Physica 7, 284 (1940).

33. M. Büttiker, E. P. Harris, R. Landauer, Phys. Rev. B 28, 1268 (1983).

34. M. H. Devoret, J. M. Martinis, D. Esteve, J. Clarke, Phys. Rev. Lett. 53, 1260 (1984). 
35. M. H. Devoret, D. Esteve, J. M. Martinis, A. N. Cleland, J. Clarke, Phys. Rev. B 36, 58 (1987).

36. T. Fonseca, P. Grigolini, Phys. Rev. A 33, 122 (1986).

37. A. I. Larkin, Y. N. Ovchinnikov, J. Low Temp. Phys. 63, 317 (1986).

38. A. O. Caldeira, A. J. Leggett, Ann. Phys. (N.Y.) 153, 445(E) (1984).

39. H. Grabert, P. Olschowski, U. Weiss, Phys. Rev. B 32, 3348 (1985).

40. H. Grabert, P. Olschowski, U. Weiss, Phys. Rev. B 36, 1931 (1987).

41. J. M. Martinis, M. H. Devoret, J. Clarke, Phys. Rev. B 35, 4682 (1987).

42. A. N. Cleland, J. M. Martinis, J. Clarke, Phys. Rev. B 37, 5950 (1988).

43. F. C. Wellstood, Ph. D. Thesis, (University of California, Berkeley, 1988)

44. O. V. Lounasmaa, Experimental Principles and Methods Below I K (Academic Press, London, 1974).

45. S. T. Ruggiero, D. A. Rudman, (Academic Press, San Diego CA, 1990),

46. C. Kittel, Introduction to Solid State Physics (John Wiley, New York, 1986).

47. M. Tinkham, Introduction to Superconductivity (R.E. Krieger, Malabar FL, 1985).

48. J. M. Jaycox, M. B. Ketchen, JEEE Trans. Mag. MAG-17, 40 (1981).

49. G. J. Dolan, Appl. Phys. Lett. 31, 337 (1977).

50. R. E. Howard, E. L. Hu, L. D. Jackel, L. A. Fetter, R. H. Bosworth, Appl. Phys. Lett. 35, 879 (1979).

51. T, van Duzer, C. W. Tumer, Principles of Superconductive Electronics and Devices (Elsevier North Holland, New York, NY, 1981).

52. M. Büttiker, R. Landauer, /BM J. Res. and Devel. 30, 451 (1986). 
53. J. B. Johnson, Phys. Rev. 32, 97 (1928).

54. H. Nyquist, Phys. Rev. 32, 110 (1928).

55. U. Geigenmulller, G. Schön, Physica (Amsterdam) 152B, 186 (1988).

56. Y. V. Nazarov, Zh. Eksp. Teor. Fiz. 95, 975 (1989).

57. S. Girvin, L. I. Glazman, M. Jonson, D. R. Penn, M. D. Stiles, Phys. Rev. Lett. 64, 3183 (1990).

58. R. Brown, E. Simanek, Phys. Rev. B 34, 2957 (1986). 